


\title{
Stable Electrospinning of Core-Functionalized Coaxial Fibers Enabled by the Minimum-Energy Interface Given by Partial Core- Sheath Miscibility
}

\author{
Shameek Vats, Manos Anyfantakis, Lawrence W. Honaker, Francesco Basoli, and Jan P. F. Lagerwall*
}

Cite This: Langmuir 2021, 37, 13265-13277

Read Online

ACCESS | Lلll Metrics \& More | 国 Article Recommendations ｜（s Supporting Information

ABSTRACT: Core-sheath electrospinning is a powerful tool for producing composite fibers with one or multiple encapsulated functional materials, but many material combinations are difficult or even impossible to spin together. We show that the key to success is to ensure a well-defined core-sheath interface while also maintaining a constant and minimal interfacial energy across this interface. Using a thermotropic liquid crystal as a model functional core and polyacrylic acid or styrene-butadiene-styrene block copolymer as a sheath polymer, we study the effects of using water, ethanol, or tetrahydrofuran as polymer solvent. We find that the ideal core and sheath materials are partially miscible, with their

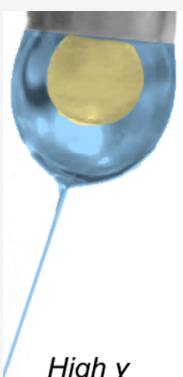

High $\gamma$

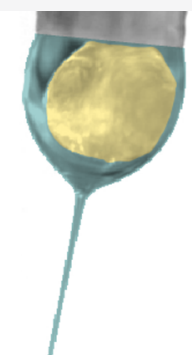

Marangoni flow

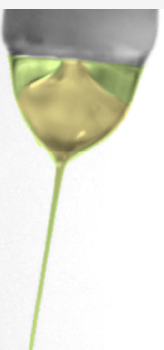

Ideal cone

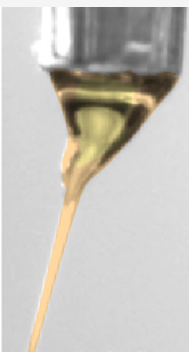

Gelation phase diagram exhibiting an inner miscibility gap. Complete immiscibility yields a relatively high interfacial tension that causes core breakup, even preventing the core from entering the fiberproducing jet, whereas the lack of a well-defined interface in the case of complete miscibility eliminates the core-sheath morphology, and it turns the core into a coagulation bath for the sheath solution, causing premature gelation in the Taylor cone. Moreover, to minimize Marangoni flows in the Taylor cone due to local interfacial tension variations, a small amount of the sheath solvent should be added to the core prior to spinning. Our findings resolve a long-standing confusion regarding guidelines for selecting core and sheath fluids in core-sheath electrospinning. These discoveries can be applied to many other material combinations than those studied here, enabling new functional composites of large interest and application potential.

\section{INTRODUCTION}

Although the idea of making fibers by electrospinning is approaching its centennial anniversary, ${ }^{1}$ it has only been in the last two decades that the technique has truly flourished. ${ }^{2-8}$ The introduction of core-sheath electrospinning using nested capillary spinnerets, often coaxial, has led to an explosion of creativity, with a diversity of functional nano- and microfibers with a variety of internal morphologies being successfully electrospun. $^{9-13}$ Pioneering contributions in demonstrating the potential of dual-phase coaxial electrospinning for making controlled core-sheath fibers were published by Sun et al., ${ }^{14}$ Yu et al., ${ }^{15}$ and $\mathrm{Li}$ and Xia. ${ }^{16}$ The latter was the first paper to spin coaxial fibers where the core was a nonpolymeric and nonvolatile liquid, thus defining a cylindrical core that could easily be removed after spinning to make hollow tubes. The authors emphasized that the core and sheath liquids must be immiscible for reliable results. This contrasted with the results of Sun et al. and $\mathrm{Yu}$ et al., which both were obtained with miscible core and sheath liquids.

While the mineral oil used as core liquid by $\mathrm{Li}$ and $\mathrm{Xia}$ was largely a sacrificial fluid, its presence ensuring tube-like fiber morphology, several subsequent electrospinning studies incorporated more precious liquids, e.g., phase change materials, ${ }^{17-19}$ liquid crystals (LC), ${ }^{20-36}$ and shear thickening fluids, ${ }^{37}$ to remain as a functional core inside the fibers. These specially selected core liquids enhance the composite fibers with dynamic and responsive performance that the sheath polymer itself is incapable of, while the coaxial fiber geometry provides a powerful means of encapsulating the liquids-which are unspinnable on their own-in a flexible form factor with high surface-to-volume ratio. Several modifications of the fundamental core-sheath electrospinning process have been explored, such as triple-phase coaxial electrospinning ${ }^{a}$, enabled by adding a third nested capillary, which can yield fibers with an intermediate layer between the innermost core and the outermost sheath. ${ }^{38-41}$ With noncoaxial electrospinning using multiple bundled capillaries inside an outer capillary that flows the sheath solution, fibers were produced with multiple core channels, consisting of identical $^{42}$ or different ${ }^{18,27}$ materials.

Received: July 8, 2021

Revised: October 20, 2021

Published: November 4, 2021 
<smiles>CC(C)(C)CC(C(=O)O)C(C)(C)C</smiles><smiles>CC(CC=CCC(C)C(C)(C)C(C)(C)C)C(C)(C)C</smiles>

Figure 1. Chemical structures of (a) poly(acrylic acid) (PAA) and (b) polystyrene-block-poly-cis-butadiene-block-polystyrene (SBS).

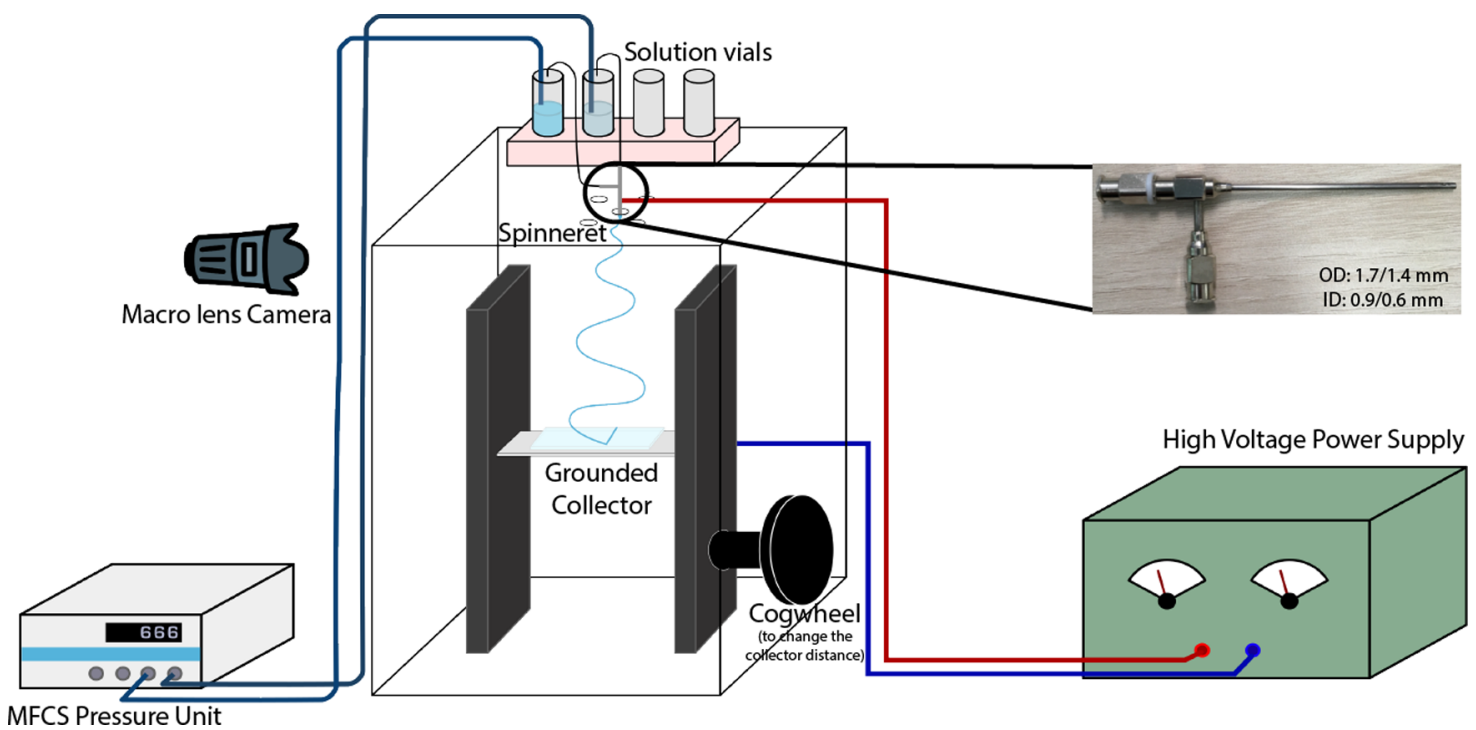

Figure 2. Schematic representation of the electrospinning setup. MFCS is the pressure control unit that controls the liquid flow. Inset: The spinneret used for the experiments.

Core-sheath fibers were also obtained using single-phase electrospinning, relying on radial phase separation during spinning. ${ }^{23,28,32,35,36}$ Using the different variations of coresheath fiber electrospinning, functional composite fibers have been produced for a variety of application scenarios, such as sustained release of drugs, ${ }^{11,40,43-52}$ growth factor, ${ }^{53,54}$ genes, ${ }^{54,55}$ or live cells; ${ }^{56-58}$ enhanced thermal insulation; ${ }^{17-19}$ sensing of volatile organic compounds; ${ }^{25,29,32,59}$ generation of wavy polymer structures; ${ }^{60}$ or sound damping. ${ }^{37}$

Despite the strong interest in core-sheath electrospinning, the answer to the critical question of whether the core and sheath liquids should be miscible or not remains elusive. The original confusion remains, with different teams publishing conflicting views on the matter, both for core-sheath electrospinning and for the closely related challenge of coresheath electrospray. Several papers reported on spinning cores and sheaths that are fully miscible, $14,15,37,41,43-46,49,53,58,61,62$ some emphasizing the importance of low interfacial tension, $\gamma_{\mathrm{cs}}$, between the two liquids. ${ }^{15,63}$ Others have maintained that cores and sheaths should be immiscible, ${ }^{16-18,30,42,55,64-66}$ often referring to the original $\mathrm{Li}$ and Xia work, which even showed evidence of loss of core-sheath structure when miscible fluids were spun. ${ }^{16}$ In our own research, we have encountered problems with both approaches, frequently failing to produce core-sheath fibers either due to excessive $\gamma_{c s}$ between immiscible liquids or due to miscible liquids without a welldefined interface fusing together, leaving no core-sheath structure in the produced fibers. Given the scarcity of publications of negative results, we believe other teams may have faced similar issues without reporting them.

The purpose of this paper is to clarify the situation by conducting a thorough and systematic investigation of coresheath interfacial phenomena and how they are affected by (im-)miscibility between the two liquids, using a liquid crystal (LC) mixture as a model fiber-functionalizing core fluid that is nonvolatile and nonpolymeric, and three representative polymer solutions for the sheath. We focus particularly on the quality of the Taylor cone, of fundamental importance to the success of any electrospinning process, since the jet that will form the fiber emanates from the Taylor cone apex. We recently demonstrated that humidity in the spinning environment can ruin the quality and stability of the Taylor cone and that certain core fluids during coaxial electrospinning can amplify this sensitivity to humidity. ${ }^{67}$ We now move the attention from the Taylor cone outside to the core-sheath interface, where we find that neither complete miscibility nor complete immiscibility is advisable: the former triggers sheath gelation and loss of core-sheath structure; the latter gives rise to core breakup in the jet, often already in the Taylor cone. The ideal is partial miscibility with a miscibility gap creating a distinct core-sheath interface, yet its interfacial tension $\gamma_{\mathrm{cs}}$ is much reduced since the two bounding phases contain the same constituents, only at different compositions. The low $\gamma_{\mathrm{cs}}$ allows an uninterrupted core flow from the inner spinneret needle to the Taylor cone apex, where it enters the jet that forms the fiber, and it also prevents the Rayleigh-Plateau instability from breaking up the continuous core within the jet. We also believe 


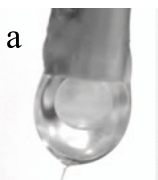

$0 \mathrm{~s}$

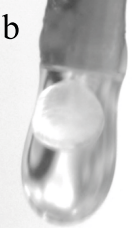

$6.0 \mathrm{~s}$ c

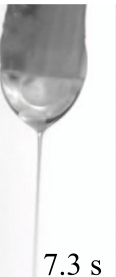

d

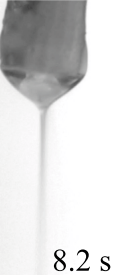

e

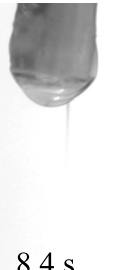

f

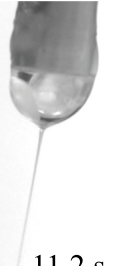

g

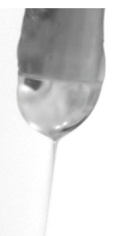

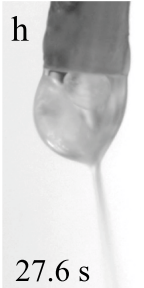

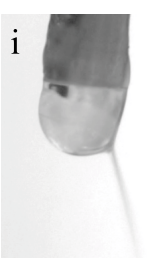

$28.0 \mathrm{~s}$

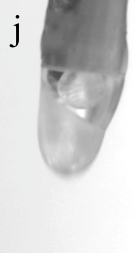

$30.4 \mathrm{~s}$

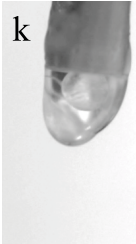

$30.5 \mathrm{~s}$
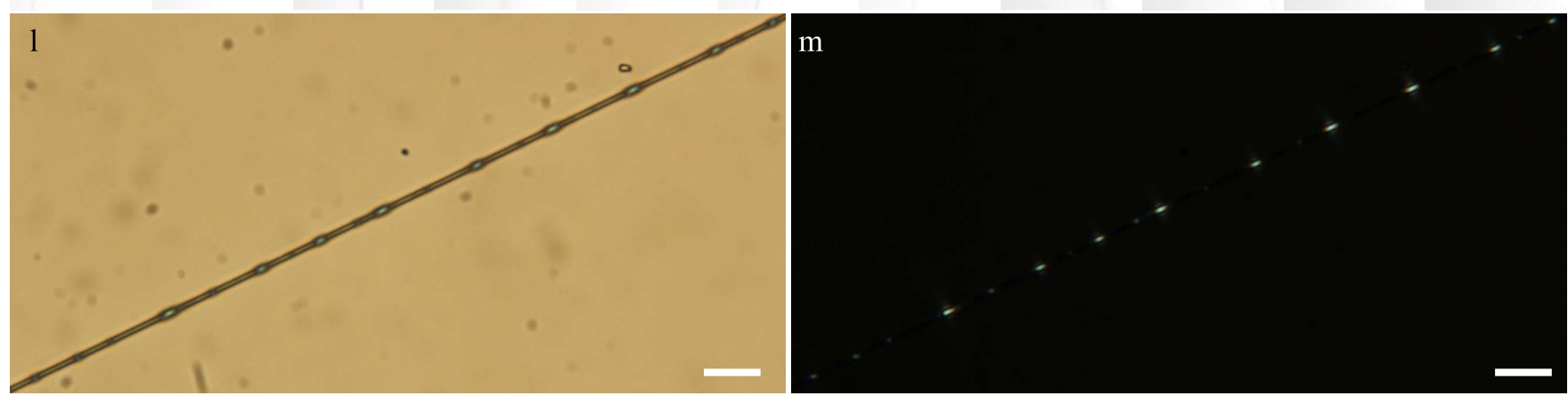

Figure 3. Top row: Taylor cone at different stages during electrospinning the LC core into an $11.5 \% \mathrm{w} / \mathrm{w}$ PAA-in-pure water sheath solution. The images are extracted from Supporting Information Movie S1, the time stamps relating to the start of the movie. The outer needle of the spinneret $(1.7 \mathrm{~mm}$ diameter) appears slightly inclined at the top of every image because the camera was not oriented perfectly; in reality, the spinneret was vertically oriented. Bottom row: polarizing optical microscope (POM) images (l: without analyzer; m: between crossed polarizers) of the bestquality fiber produced during this experiment, exhibiting regularly spaced beads filled with LC. Scale bars are $20 \mu \mathrm{m}$.

that solutal Marangoni flow, to the best of our knowledge not previously discussed in the context of core-sheath electrospinning, can have a highly disruptive influence, and we find that the problem can be avoided by premixing a small fraction of sheath solvent into the core prior to electrospinning.

\section{EXPERIMENTAL SECTION}

Polymer Solutions and Liquid Crystals. Poly(acrylic acid) (PAA; $\overline{M_{\mathrm{w}}} 450 \mathrm{~kg} / \mathrm{mol}$, Figure 1a), a polymer soluble in both water and ethanol, was purchased from Sigma-Aldrich and either dissolved in anhydrous ethanol (purchased from VWR) to prepare a $10 \% \mathrm{w} / \mathrm{w}$ solution or in ultrapure deionized water (Sartorius Arium system, resistivity $18.2 \mathrm{M} \Omega \cdot \mathrm{cm})$ to make an $11.5 \% \mathrm{w} / \mathrm{w}$ PAA-water solution. Polystyrene-block-poly-cis-butadiene-block-polystyrene (SBS; 30\% w/ w styrene; $\overline{M_{\mathrm{w}}} 140 \mathrm{~kg} / \mathrm{mol}$, Figure $1 \mathrm{~b}$ ) was also purchased from Sigma-Aldrich and dissolved in tetrahydrofuran (THF, from SigmaAldrich) to prepare a $10 \% \mathrm{w} / \mathrm{w}$ solution. For the core material, we used the multicomponent nematic liquid crystal mixture RO-TN 651 (proprietary composition), sourced from F. Hoffman-La Roche (Basel, Switzerland), on its own or mixed with $10 \% \mathrm{w} / \mathrm{w}$ of ethanol. We measured the surface tension of pure RO-TN 651 to be $32.33 \pm$ $0.02 \mathrm{mN} / \mathrm{m}$ (at $20{ }^{\circ} \mathrm{C}$ ); the surface tension of ethanol (at $20{ }^{\circ} \mathrm{C}$ ) is $22.31 \mathrm{mN} / \mathrm{m}^{68}$ We could not measure the surface tension of the mixture of RO-TN 651 with $10 \% \mathrm{w} / \mathrm{w}$ ethanol because this mixture wets the needle used to make a pendant drop (even when a needle made from poly(tetrafluoroethylene), PTFE is employed). Nevertheless, we expect that the surface tension of the ethanol/RO-TN 651 mixture falls within the range of $22-32 \mathrm{mN} / \mathrm{m}$, bounded by the values corresponding to the pure components. All materials were used as received without further purification.

Electrospinning Parameters. The dual-phase spinneret used for electrospinning, consisting of coaxially mounted stainless steel needles (external/internal diameter of the inner needle: $0.9 / 0.6 \mathrm{~mm}$; of the outer needle: $1.7 / 1.4 \mathrm{~mm}$ ), was purchased from Y-Flow. The outer needle of the spinneret has dents to keep the inner needle in the center and, at the same time, to provide an ohmic contact between the two needles that ensures they are at the same electrical potential. The spinneret was stored in ethanol when not in use and, prior to and after experiments, was thoroughly rinsed with fresh $96 \% \mathrm{w} / \mathrm{w}$ ethanol to remove any material residues. Before starting the electrospinning process, the spinneret was carefully dried to avoid any possible cross contamination from the lower-grade ethanol used for cleaning. This was achieved by flushing the spinneret with compressed air and storing it at $25{ }^{\circ} \mathrm{C}$ for a few hours.

Figure 2 shows a schematic representation of the electrospinning setup. It is housed inside an acrylic box, with a mobile collector wrapped in aluminum foil and the spinneret inserted with vertical needle orientation from the top. The fluids are pumped to the respective spinneret needle through tubes connected to fluid vials pressurized by a microfluidic pressure unit (Fluigent, model MFCSEZ, maximum pressure 1034 mbar, uncertainty $\pm 0.3 \mathrm{mbar}$ ), and the electrical potential of the spinneret is controlled by connecting the outer needle to a high-voltage power supply ( $\gamma$ High Voltage, model ES30R-5W/DAM/RS232). The Taylor cone was imaged using a macro lens (Tokina AT-X Pro) mounted on a camera (Pixelink D755). Representative still frames were extracted from the movies and then digitally enhanced for clarity using "Adjust image" in Keynote (Apple), setting Saturation at $-100 \%$, the right-most Levels parameter to $53 \%$, and the middle one to $40 \%$ (Figures 3, 5, and 6) or 47\% (Figure 7). In Figures 3, 5, and 6, Exposure and Shadows were additionally adjusted to $100 \%$.

Fibers were collected freely hanging on a copper wire frame and on hydrophobized glass microscopy slides to avoid wetting and collapse of the filled fibers. ${ }^{27,69}$ These slides were prepared by cleaning $25 \mathrm{~mm}$ $\times 75 \mathrm{~mm}$ borosilicate glass microscopy slides (Carl Roth) with alternating rinses of isopropanol and ultrapure deionized water before surface activation with a handheld corona generator for at least $30 \mathrm{~s}$. The plasma-treated slides were then immediately immersed in an aqueous solution of $2 \% \mathrm{v} / \mathrm{v} \mathrm{N}, \mathrm{N}$-dimethyl-[N-octadecyl-3aminopropyl]trimethoxysilyl chloride (DMOAP, 42\% in methanol, Sigma-Aldrich) and allowed to stand for at least $15 \mathrm{~min}$, with gentle shaking halfway through the soaking procedure to ensure that the solution adequately coated and functionalized the glass slides. The slides were then removed from the treatment solution, rinsed several times with deionized water, and dried under vacuum at $120^{\circ} \mathrm{C}$ for at least $30 \mathrm{~min}$.

Establishment of Phase Diagram between RO-TN 651 and Ethanol. Vials of volume $2 \mathrm{~mL}$ were half-filled with each mixture, prepared by measuring out target volumes of first LC and then ethanol using Eppendorf pipettes and weighing the sample after each addition step. To minimize evaporation of ethanol and condensation of water, the vials were closed immediately after addition of the 


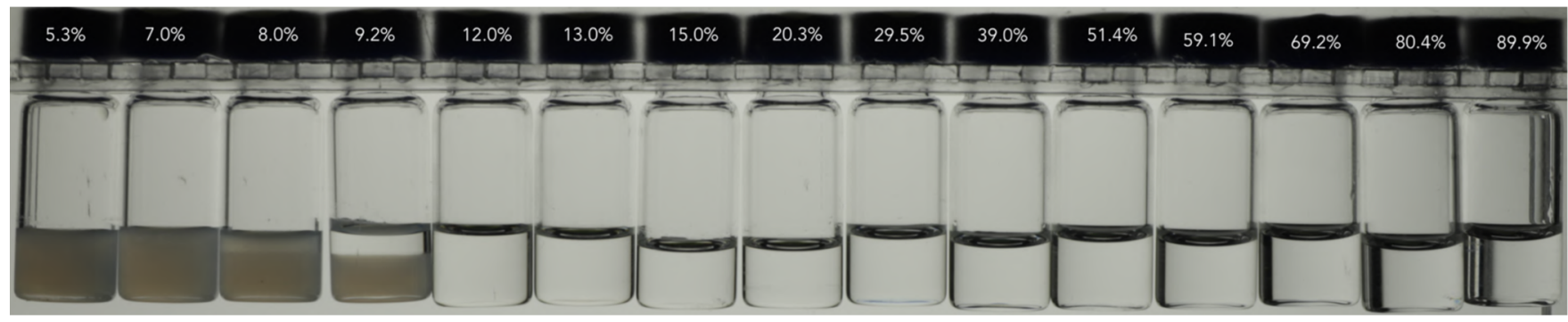

Figure 4. Sequence of vials with RO-TN 651 and gradually increasing concentration of anhydrous ethanol from left to right; the indicated percentages at the top refer to the mass fraction of ethanol. Because the nematic phase is turbid and sinks to the bottom, it is easy to recognize, although the phase separation had not completed in the $8.0 \%$ sample at the time of photography (about 20 min after the last sample preparation). We conclude the existence of a miscibility gap extending from around $\sim 7.5 \% \mathrm{w} / \mathrm{w}$ to around $\sim 11 \% \mathrm{w} / \mathrm{w}$ ethanol.

ethanol. After all samples had been prepared, each vial was shaken vigorously for about $1 \mathrm{~min}$ on a vortex mixer to ensure complete mixing. After this, the series of sealed vials were left to stand overnight in an air-conditioned lab with the temperature set to $21^{\circ} \mathrm{C}$, before the photograph shown in Figure 4 was captured.

Optical Characterization. Once collected, the fibers were optically characterized using a polarizing optical microscope (POM; Olympus BX-51) with a camera (Olympus DP73). POM characterization was carried out in transmission mode between crossed polarizers or with the analyzer removed.

Interfacial Tensiometry. Interfacial tension measurements were performed using a pendant drop tensiometer (Goniometer OCA 15EC from Dataphysics). The density of the solutions for interfacial tensiometry was measured using a Mettler Toledo DE45 Delta range densitometer. Both density and interfacial tension measurements were performed at room temperature.

\section{RESULTS AND DISCUSSION}

Coaxial Spinning with Aqueous PAA Sheath and LC Core: Impact of Excessive Interfacial Tension. When attempting to electrospin the LC RO-TN 651, which has negligible miscibility with water, as a core inside the PAAwater sheath solution, the relatively high $\gamma_{\mathrm{cs}}$ (measured to be $9.13 \pm 0.3 \mathrm{mN} / \mathrm{m}$ at $20^{\circ} \mathrm{C}$; see Supporting Information Movie S5) causes significant problems, as seen from a detailed frameby-frame analysis of Supporting Information Movie S1, showing the Taylor cone dynamics during a run with flow rates optimized for maximum fiber filling. Representative still frames are shown in Figure $3 \mathrm{a}-\mathrm{k}$. It is still possible to spin fibers with encapsulated LC in this way (Figure $31-m$ ), but we cannot get a continuous LC core and it is a very lossy process since the required overfilling of the Taylor cone with LC (to be explained below) means that the majority of the LC pumped to the spinneret never makes it into the jet.

We initially pump only the sheath solution, starting the injection of the LC core solution once a stable PAA-water Taylor cone with consistent spinning has been established. As seen at the beginning of Supporting Information Movie S1 and in Figure 3a, the LC pumped from the inner needle forms a nearly spherical droplet inside the external sheath solution, hovering far above the Taylor cone apex from which the jet is ejected. The fibers produced at this stage of the process are thus devoid of LC since no LC makes it into the jet. Because we use a high LC flow rate, the inner droplet and the overall Taylor cone continuously grow in volume and, about $2 \mathrm{~s}$ into the movie, the jet stops: most likely, there is too large a voltage drop from the spinneret to the bottom of the droplet at this size. Now both sheath and core droplets grow until $\sim 6 \mathrm{~s}$ into the movie, when the sheath droplet rapidly elongates before it is cleaved (Figure $3 \mathrm{~b}$ ). The cleavage process detaches the entire LC droplet from the spinneret, and most of it—but not all-leaves the Taylor cone together with the detached sheath solution.

The remainder of the LC separated from the spinneret forms a small droplet near the bottom of the Taylor cone, from which a stable jet is again ejected. The suction from the jet pulls the bottom LC droplet toward it until that in Figure $3 \mathrm{c}$ connects to the Taylor cone apex such that the jet is now injected with LC. In the meantime, a new top droplet of freshly injected LC has started growing from the spinneret. Once this has become large enough to touch and merge with the bottom LC droplet, we have a brief moment with a single LC volume that extends continuously from the inner spinneret needle all the way to the jet, thus yielding an ideal coaxial Taylor cone (see Figure 3d). However, this shape of the LC volume does not minimize the core-sheath interface area, and therefore the interfacial tension $\left(\gamma_{\mathrm{cs}}=9.13 \pm 0.3 \mathrm{mN} / \mathrm{m}\right.$; see the Supporting Information) renders this an unstable equilibrium. The continuous LC flow very quickly collapses into a geometry with a bottom LC drop again detached from the spinneret needle; see Figure 3e. At the same time, the jet moves from the bottom of the Taylor cone to the boundary between LC and sheath solution.

The lower LC droplet-which is now larger than before due to the merger with the new LC-moves to the left, detaching from the jet, which thus again contains no LC (Figure 3f). A new droplet grows from the inner spinneret needle until it merges with the lower LC droplet (Figure 3i), which thereby acquires a size large enough that it extends past the cone apex, hence now LC is again fed into the jet. This cycle of new LC droplet growing from the spinneret needle until it is large enough to merge with the LC droplet residing at the bottom of the Taylor cone, thereby separating from the spinneret and leaving an increasingly larger lower LC droplet, repeats itself another five times, the last two steps shown in Figure $3 \mathrm{~h}-\mathrm{i}$. Throughout this stage, the jet is continuously fed with LC as the Taylor cone apex is fully covered by the lower LC droplet. However, since the process requires overfeeding of the Taylor cone with LC, the process makes the inner LC droplet, and thus the overall Taylor cone, increasingly heavier, and in Figure $3 j$, another cleavage event occurs, removing most of the LC from the Taylor cone. Again, a fraction is left at the bottom of the Taylor cone (Figure $3 \mathrm{k}$ ) and the full cycle repeats itself.

While this trick of overfeeding the Taylor cone with LC thus leads to core injection into the jet a large fraction of the time, it comes at the cost of very significant loss of LC every time a droplet is pinched off from the Taylor cone, in addition to the problems that the macroscopic drop may cause if it lands on 

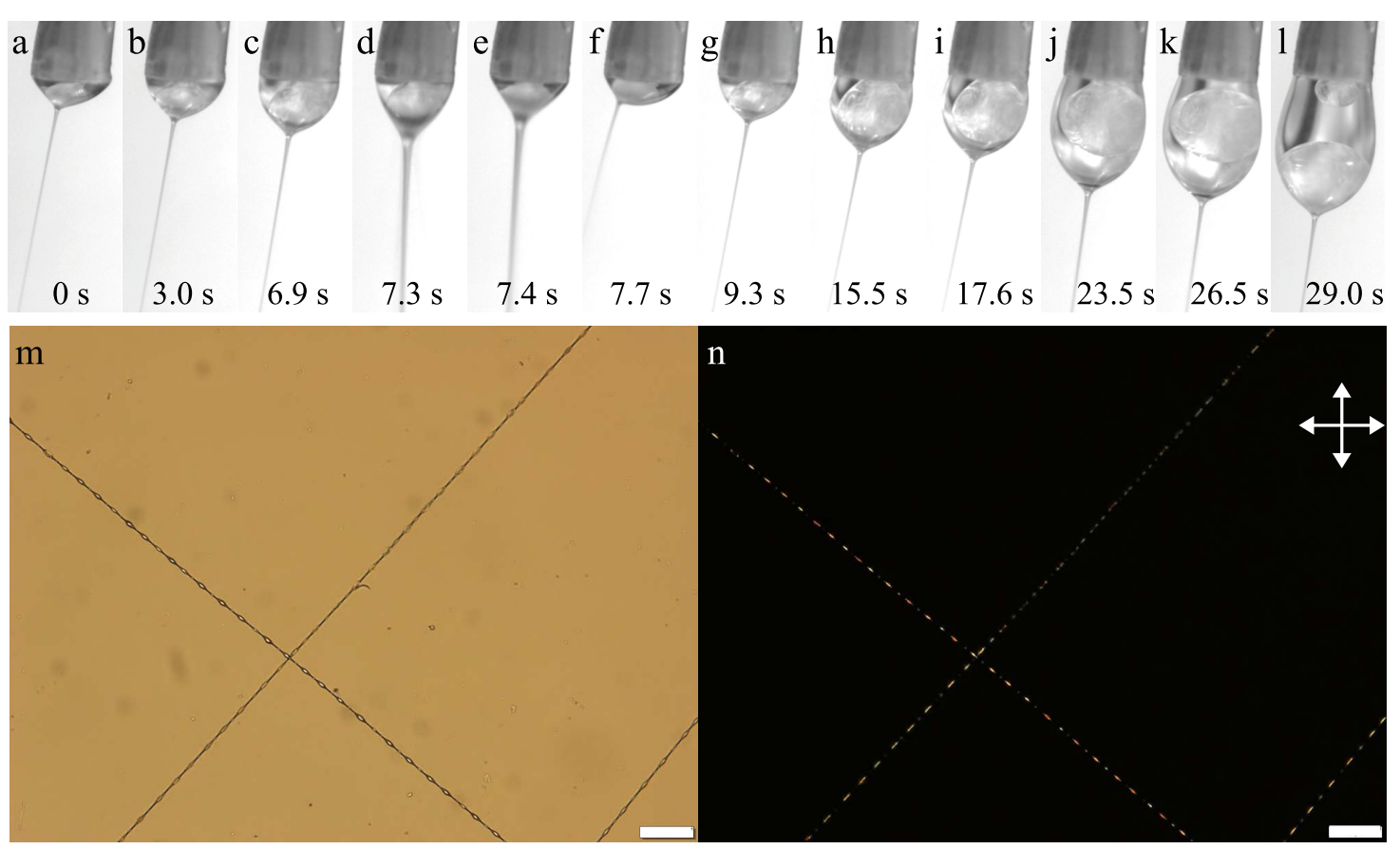

Figure 5. Top row: Taylor cone at different stages during electrospinning the LC core into a $10 \% \mathrm{w} / \mathrm{w}$ PAA in pure ethanol sheath solution. The images are extracted from Supporting Information Movie S2, the time stamps relating to the start of the movie. The outer needle of the spinneret $(1.7 \mathrm{~mm}$ diameter) appears at the top of every image, slightly inclined because the camera was not oriented perfectly; in reality, the spinneret was vertically oriented. Bottom row: POM images ( $\mathrm{m}$ : without analyzer; $\mathrm{n}$ : between crossed polarizers, as indicated by double-headed arrows) of the best-quality fiber produced during this experiment, exhibiting regularly spaced beads filled with LC. Scale bars are $50 \mu \mathrm{m}$.

the fiber mat. Note that horizontal electrospinning cannot be used in this mode, since we rely on gravity to push the detached lower LC droplet on top of the jet. Moreover, even during the period when the jet is fed with LC, the relatively high $\gamma_{\mathrm{cs}}$ continues to cause problems within the jet, triggering an internal Rayleigh-Plateau instability that breaks up the continuous core into a train of discrete LC droplets. The result is that the best fibers produced with this core-sheath combination are beaded, with discrete pockets of LC regularly spaced along the fibers; see the example in Figure 31-m.

Replacing Water with Ethanol in the Sheath Solution. While RO-TN 651 is practically insoluble in water, the mixture is partially soluble in ethanol. Figure 4 shows that about $7.5 \%$ $\mathrm{w} / \mathrm{w}$ anhydrous ethanol destabilizes the nematic phase at room temperature, and the miscibility gap between the ethanol-poor nematic phase and ethanol-rich isotropic phase extends to between 9.2 and $12.0 \% \mathrm{w} / \mathrm{w}$. Although we cannot establish the mole percentages since we do not know the composition of the commercial RO-TN 651 mixture, the miscibility gap appears to be somewhat narrower than that of ethanol and the commonly used single-component $\mathrm{LC} 5 \mathrm{CB}$, which at room temperature extends from $\sim 13$ to $\sim 23 \mathrm{~mol} \%{ }^{70}$ At no point do we see two isotropic phases in coexistence in Figure 4. The partial miscibility of RO-TN 651 and ethanol, and the miscibility gap starting at low ethanol concentrations, render an experiment using PAA dissolved in ethanol as sheath and RO-TN 651 as core highly interesting. The miscibility gap ensures that a transient, yet well-defined interface exists between core and sheath, even if they start mixing, as the core is continuously replenished with fresh LC from the spinneret. At the same time, the nonzero miscibility means that the phases on both sides of the interface contain the same chemical substances, only at different concentrations. We can thus expect a much lower $\gamma_{\mathrm{cs}}$ than the ca. $9 \mathrm{mN} / \mathrm{m}$ measured for the case where water-dissolved PAA is the sheath solution. We indeed confirm this while attempting to measure the interfacial tension of a pendant RO-TN 651 drop in a bath of a PAA/ethanol solution. Although a stable RO-TN 651 drop cannot be formed at equilibrium, a drop with a well-defined fluid interface is formed while the LC phase is ejected from the needle into the polymer solution. When the LC flow is stopped, the LC drop slightly increases in size (presumably due to ethanol from the bath mixing with the LC) and the boundary between the two fluids becomes decreasingly sharp (see Figure S1, Movie S6, and the corresponding discussion).

The result can be seen in Supporting Information Movie S2, with representative still images collected in Figure 5, showing that the situation is still far from ideal. Initially (Figure 5a-c), the inner LC core can be distinguished, as being surrounded by an increasingly turbid mixed phase that grows with time from bottom to top of the Taylor cone. During this stage, the core LC appears to be disconnected from the jet, leading to its continuous increase in volume until it connects to the jet in Figure 5d. The jet suddenly broadens greatly as much of the collected LC is ejected from the Taylor cone which rapidly diminishes in size $(\mathrm{d}-\mathrm{f})$. Around $10 \mathrm{~s}$ into the movie (Figure $5 \mathrm{~g}-\mathrm{h})$, flow patterns are clearly seen, and the LC droplet appears to disconnect from the jet. The LC is still attached to the spinneret, however, so the droplet hovers further and further above the apex of the Taylor cone as the latter continues to grow in size ( $i, j)$. The LC eventually detaches from the spinneret $26.5 \mathrm{~s}$ into Supporting Information Movie S2 (Figure 5k), settling at the bottom of the Taylor cone in Figure 5l, after which the jet is again fed with LC. The situation now resembles that of Figure 3, with a bottom LC droplet resting on top of the jet ejection point and a top droplet attached to the spinneret needle growing in size until the two 

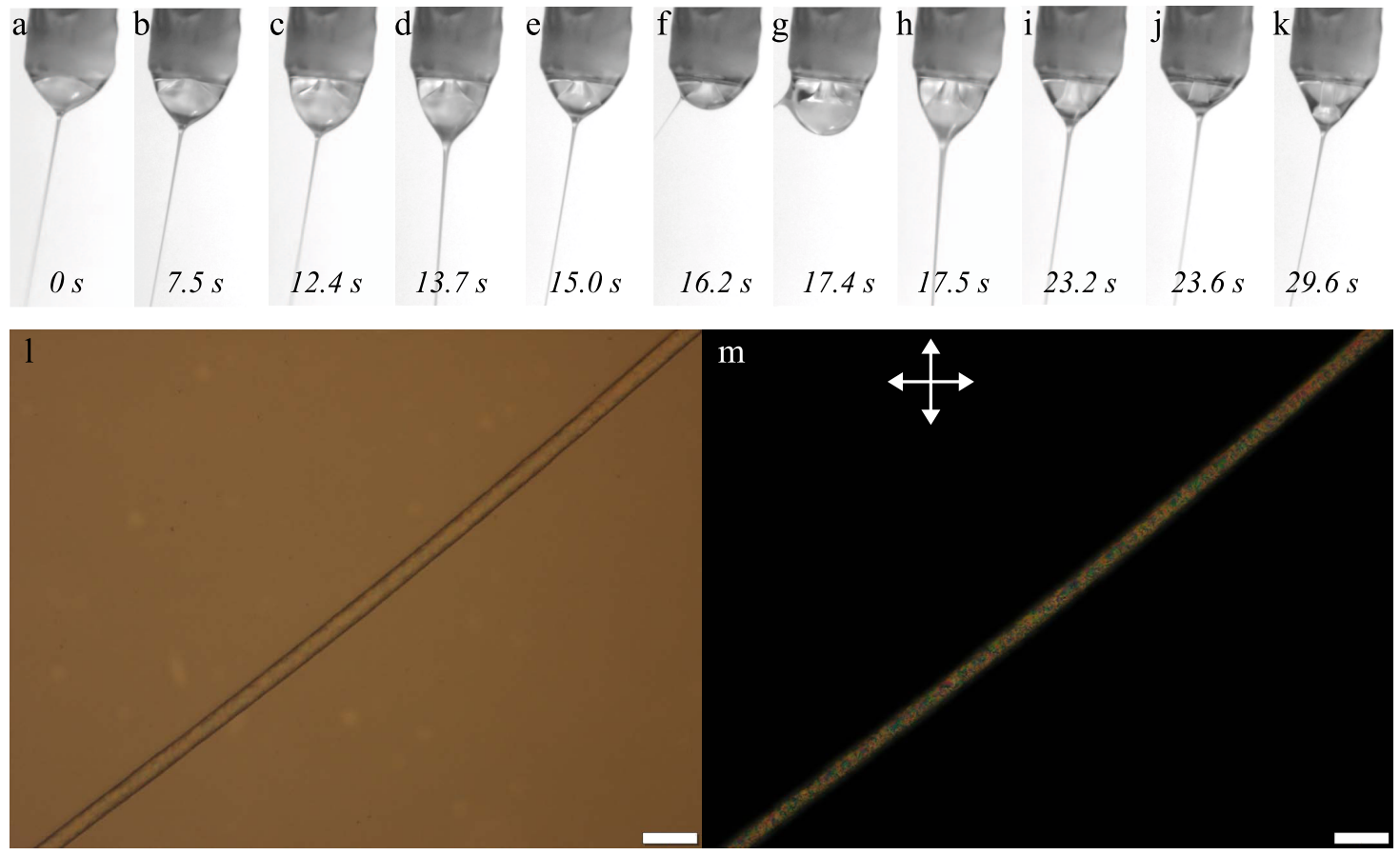

Figure 6. Top row: Taylor cone at different stages during electrospinning a core of LC with $10 \% \mathrm{w} / \mathrm{w}$ of ethanol added into a $10 \% \mathrm{w} / \mathrm{w}$ PAA in pure ethanol sheath solution. The images are extracted from Supporting Information Movie S3, the time stamps relating to the start of the movie. The outer needle of the spinneret $(1.7 \mathrm{~mm}$ diameter $)$ appears at the top of every image, slightly inclined because the camera was not oriented perfectly; in reality, the spinneret was vertically oriented. Bottom row: POM images (m: without analyzer; $\mathrm{n}$ : between crossed polarizers, as indicated by double-headed arrows) of the best-quality fiber produced during this experiment, exhibiting continuous filling of LC. Scale bars are 50 $\mu \mathrm{m}$.

LC volumes merge and/or the Taylor cone becomes so large that it detaches from the spinneret.

We believe that the problems seen in this experiment arise because $\gamma_{\mathrm{cs}}$ is not constant within the Taylor cone: we expect a continuous gradient in $\gamma_{\mathrm{cs}}$ from the top of the spinneret, where pure LC comes in contact with pure PAA/ethanol solution, to the point further down along the core-sheath interface where enough mixing of the two liquids should have occurred to reach the miscibility gap. From this level and downward, we can expect an extremely low $\gamma_{\mathrm{cs}}$ between the coexisting nematic and isotropic phases. As a result of this spatial variation in $\gamma_{\mathrm{cs}}$ along the axis defined by the spinneret, we anticipate that the solutal Marangoni effect ${ }^{71,72}$ sets up a new internal flow along the internal LC-polymer solution interface. Since this flow is directed from low to high $\gamma_{\mathrm{cs}}$, it is counter-directed to the topdown flow from the spinneret (assuming that the LC droplet is in contact with both the spinneret and the apex of the Taylor cone). We thus get a circular flow pattern around the coresheath interface, with the innermost LC moving downward while the interface is moving upward, promoting mixing and disturbing the interface, in turn causing new Marangoni stresses. This circular flow can be visualized in Supporting Information Movie S2.

In parallel, we expect to have another Marangoni effectdriven flow at the outer surface of the Taylor cone (i.e., at the air-PAA/ethanol interface). Cooling due to evaporation of ethanol leads to water condensation from the atmosphere, ${ }^{67}$ rendering the surface tension between sheath solution and air, $\gamma_{\mathrm{sa}}$, higher at the bottom of the air-PAA solution interface than at the top, where fresh ethanol solution without water emerges from the spinneret. This flow is from the top to the bottom (along the outer interface), thus reinforcing the natural flow within the sheath solution. In summary, we thus have the pressure-induced downward-directed flow at the very center of the Taylor cone where fresh LC is injected as core liquid; upward-directed flow at the core-sheath interface thanks to the solutal Marangoni effect; and downward-directed flow at increased speed along the Taylor cone outside, given by the sum of the pumped-out sheath solution and the thermal Marangoni effect. We can thus expect a highly complex process with multiple vortices within the Taylor cone, in combination with mixing of water into the sheath solution from the outside and a certain degree of mixing with core at the inside. Elaborate flow visualization experiments are needed to draw clear conclusions about the features of both the solutal and thermal Marangoni flows (e.g., their strength) and their interplay with the other hydrodynamic patterns inherently involved in the electrospinning process; while such experiments would be highly interesting, they are beyond the scope of this study.

The produced fibers are again beaded, with LC within the beads; see Figure $5 \mathrm{~m}, \mathrm{n}$. This suggests that the mixing of ethanol from the sheath into the LC core is not fast enough to significantly reduce $\gamma_{\mathrm{cs}}$ below the level where it triggers the Rayleigh-Plateau instability within the jet prior to sheath solidification. Indeed, when preparing the experiment in Figure 4 , we noticed that diffusion of LC and ethanol across the nematic-isotropic phase boundary is slow, motivating the active vortex mixing. Even if the Marangoni effects induce some active mixing in the Taylor cone, this is not enough to give the core and sheath fluids forming the compound jet so much of each other's constituents that $\gamma_{\text {cs }}$ loses its impact. To reach that state, we need to adjust the core composition already prior to spinning. 
Optimum Spinning Conditions by Adding Ethanol also to the Core. To reduce the impact of Marangoni flow, we add $10 \% \mathrm{w} / \mathrm{w}$ of ethanol to RO-TN 651 and fill this into the vial for core liquid. Note that this has brought us more than halfway into the miscibility gap; hence, we can expect a minimum $\gamma_{\mathrm{cs}}$ between the nematic phase and its coexisting isotropic phase. It also means that we have phase separation in the reservoir from which the core liquid is pumped, but we know that we are pumping only the nematic phase as core because we use pneumatic pumping rather than syringe pumps, and we place the tip of the needle taking the core liquid at the bottom of the vial. Based on the experiment in Figure 4, we can estimate the amount of ethanol in the nematic phase in the miscibility gap, which is our core liquid in this new experiment, to be about $7.5 \% \mathrm{w} / \mathrm{w}$. The advantage of this approach is that $\gamma_{\mathrm{cs}}$ is significantly reduced already when the two liquids first come into contact, a slight further reduction happening on the way down from the spinneret orifice as LC diffuses out into the ethanol-PAA solution, bringing its composition closer to that of the equilibrium isotropic phase bounding the miscibility gap. A gradient in $\gamma_{\mathrm{cs}}$, inducing Marangoni flow, thus still exists, as visible in Supporting Information Movie S3 (showing the full experiment) and in Figure 6 (summarizing the key observations), but it is not strong enough that the induced flow can disrupt the coaxial spinning. The effect could probably be canceled out completely by adding LC to the sheath solution until it has the composition of the isotropic phase in the miscibility gap, but as the LC is not a good solvent for PAA, this would cause other problems. We find that tuning the core composition to that of the miscibility gap boundary, while keeping the sheath solution free of $\mathrm{LC}$, is sufficient to produce good coaxial fibers.

From an applied point of view, the single most important observation in Supporting Information Movie S3 is that the LC flow is uninterrupted throughout the entire experiment: we can easily confirm a continuous stream of LC from the inner spinneret needle orifice to the Taylor cone apex, and from there into the jet, in every frame. This is despite the fact that we exchanged the collector about halfway into the movie, causing significant temporary alterations of the electric field profile with strong shape changes of the overall Taylor cone shape. However, the experiment actually contains much more information, revealing important data on the phase separation and the interfaces present within the Taylor cone, as the following detailed analysis highlights.

When the core enters the Taylor cone, it quickly builds up a turbid volume from the bottom of the Taylor cone, filling most of it in Figure 6a. Over the next few seconds, the overall Taylor cone grows vertically downward, the lower turbid LC volume retaining a roughly constant size and moving downward, with a narrower stream of core flow connecting it to the inner spinneret needle; see Figure $6 \mathrm{~b}-\mathrm{d}$. At the same time, it becomes increasingly clear that a near-horizontal boundary between two isotropic phases exists, separating the nearly pure sheath solution freshly emerged from the outer spinneret needle from the lower Taylor cone part, which has an air interface with weaker curvature just below the boundary, best seen in panel (b). We thus have two phase boundaries in the Taylor cone: a lower one between nematic and isotropic phases, rich and poor in RO-TN 651, respectively, and an upper boundary between two phases that are both isotropic. The latter type of phase separation is not present in the mixtures of RO-TN 651 and pure anhydrous ethanol, as seen in Figure 4. We conjecture that the presence of PAA in the sheath solution creates a small second miscibility gap, between the pure PAA-in-ethanol sheath solution emerging from the spinneret and the slightly LC-enriched isotropic ethanol-PAA solution that is in equilibrium with the nematic core. Another possibility is that water condensing onto the Taylor cone from the air $^{67}$ shifts the phase diagram to such an extent that an isotropic-isotropic phase separation takes place at very low LC concentration, similar to what is seen with $5 \mathrm{CB}-$ ethanol solutions. ${ }^{70}$ Importantly, with RO-TN 651 as core, neither phase boundary destabilizes the spinning process, so the interfacial tension of each boundary must be very low.

The LC flow is generally clearer at the top than at the bottom, probably because of shear alignment of the director as the LC exits the spinneret, but this alignment is lost when the core flow hits the boundary to air near the Taylor cone apex, leading to strong light scattering. The boundary between the isotropic phases moves upward and is almost flush with the spinneret in panels (c)-(d), but, in panel (e), it has moved down a bit. Around this time in the experiment, we switched to a different collector, temporarily yet considerably distorting the electric field. As a result, the Taylor cone gets smaller and, in panel (f), the symmetry is broken, the jet moving to one side. The Taylor cone distortion reaches its extreme situation in panel $(\mathrm{g})$. In panel (h), the new collector is in place and the jet moves down to the bottom of the Taylor cone, which is now nearly cylindrically symmetric. Over the next few seconds, the Taylor cone shrinks somewhat again, adopting a true cone shape in panel (i), where the horizontal upper phase boundary is easily distinguished. The Taylor cone fluctuates slightly in size after the collector switch, reaching its minimum size in panel $(j)$. In panel $(k)$, the final steady-state situation is shown, with a Taylor cone that is largely conical in shape, a distinct horizontal isotropic-isotropic boundary just below the spinneret orifice, and an LC core flow that is almost cylindrical throughout the top two-thirds of the Taylor cone, broadening only near the bottom.

Throughout the whole process, the core flow is uninterrupted, and the jet only experiences the moving-around at the height of the disturbance due to the collector change. Apart from this moment (lasting about a second), a continuous core-sheath jet is ejected from the apex of the Taylor cone. Also the produced fibers are continuously-and richly-filled with LC (Figure 61-m), demonstrating that $\gamma_{\mathrm{cs}}$ is too low to trigger the Rayleigh-Plateau instability within the jet. Our attempts to measure $\gamma_{\mathrm{cs}}$ of this system using a pendant ethanol/RO-TN 651 drop in a PAA/ethanol bath failed: a stable drop could not be formed (see Supporting Information Movie S8 and the discussion in the Supporting Information). Nevertheless, the very low effective interfacial tension is clear from the nonminimizing behavior of the interface. Since any remaining ethanol in the core is easily evaporated after spinning, its presence during the spinning process will not affect the behavior of the LC core when the fibers are used.

Case of Complete Core-Sheath Miscibility. If one only considers the impact of $\gamma_{\mathrm{cs}}$, the best option might appear to be to eliminate the interface entirely by choosing a core and a sheath that are fully miscible, as then a smooth concentration gradient can form all the way from pure sheath to pure core without any discontinuity. Without an interface, there is also no interfacial tension and the problems encountered so far will not arise. However, this option leads to other problems: first of all, the loss of core-sheath structure that already Li and Xia 


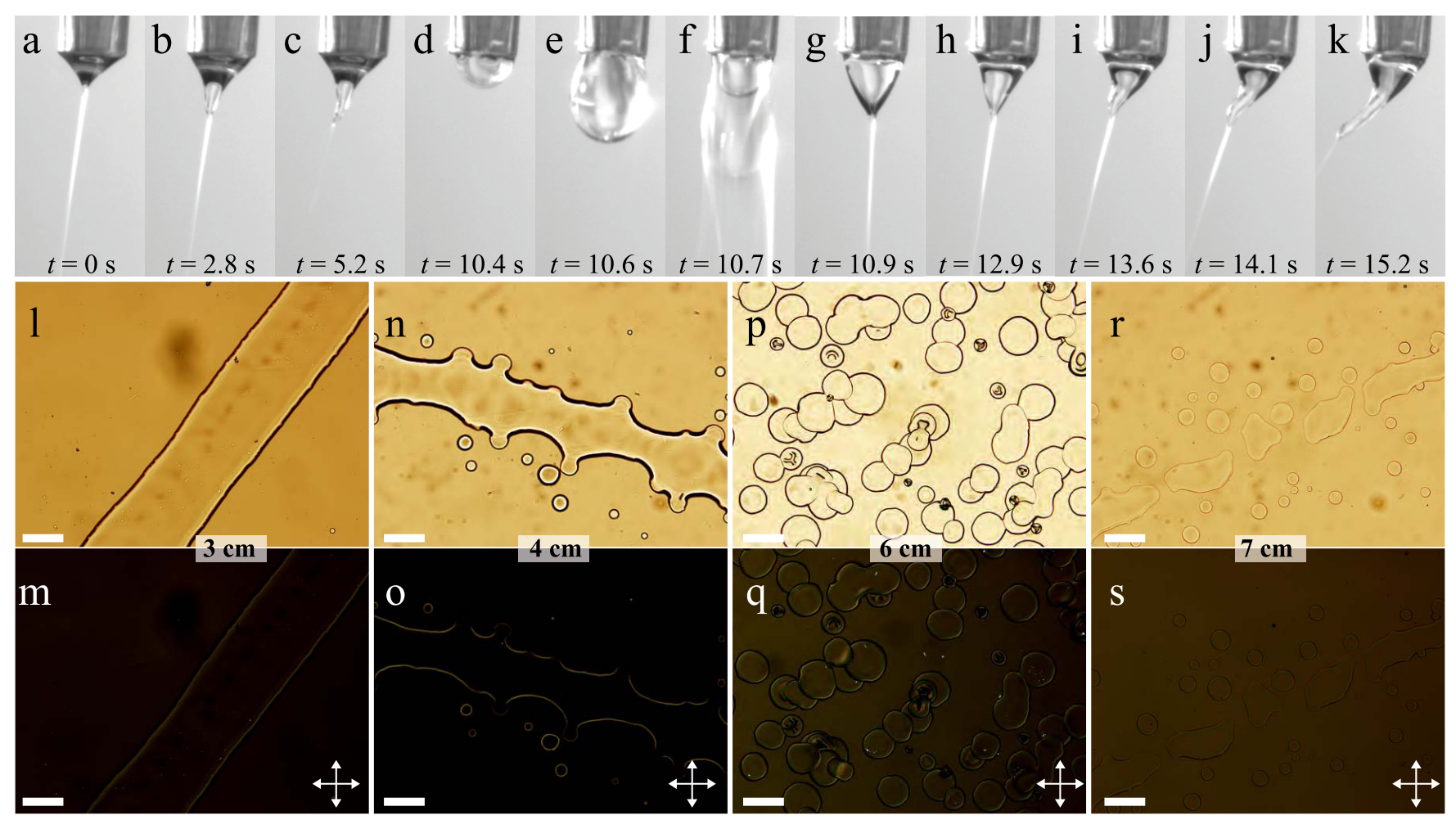

Figure 7. Top row: Taylor cone at different stages during electrospinning a core of RO-TN 651, with $10 \% \mathrm{w} / \mathrm{w}$ added THF, into a $10 \% \mathrm{w} / \mathrm{w}$ SBS in THF sheath solution. The images are extracted from Supporting Information Movie S4, the time stamps relating to the start of the movie. The outer needle of the spinneret ( $1.7 \mathrm{~mm}$ diameter) appears at the top of every image, slightly inclined because the camera was not oriented perfectly; in reality, the spinneret was vertically oriented. Bottom rows: POM images (l, n, p, r: without analyzer; $\mathrm{m}, \mathrm{o}, \mathrm{q}$, s: between crossed polarizers, as indicated by double-headed arrows) of the spinning product collected at 3, 4, 6, and $7 \mathrm{~cm}$ below the spinneret, respectively. Scale bars are $10 \mu \mathrm{m}$.

noted in their pioneering work. ${ }^{16}$ This is particularly critical when-as in the present work-the core is a low molar mass liquid, since then we cannot rely on the low miscibility of polymeric solutes to prevent or at least slow down core-sheath mixing. Even more critically, if core and sheath are fully miscible but the core is not a good solvent for the sheath polymer (typically the case with functional core materials like LCs), then the loss of sheath solvent into the core and core liquid moving into the sheath will rapidly deteriorate the quality of the sheath solution from the perspective of dissolving the polymer. The core effectively becomes an internal coagulation bath, as used in traditional wet spinning to rapidly solidify the polymer. If the sheath solvent is volatile, the polymer concentration in the sheath solution also rapidly decreases and/or water is condensed from the air, adding yet another nonsolvent for many polymers. The combined result is that the Taylor cone is strongly distorted and often clogged within seconds or minutes, disrupting the spinning process.

To demonstrate these problems, we choose styrenebutadiene-styrene (SBS) block copolymer dissolved in tetrahydrofuran (THF) as a relevant example of a sheath solution that is highly miscible with RO-TN 651. This sheath is interesting because several groups have successfully electrospun SBS dissolved in THF (and co-solvents) into highly stretchable elastomeric fiber mats, ${ }^{73}$ which may then serve as a basis for stretchable electronic composites, ${ }^{74}$ light-emitting diodes (LEDs), ${ }^{75}$ or wearable organic vapor sensors. ${ }^{76} \mathrm{We}$ have ourselves tried to use SBS as a sheath for LC corefunctionalized fibers (with and without addition of dimethylformamide (DMF) as a co-solvent), but without success. In the experiment shown in Supporting Information Movie S4 and Figure 7 , the reasons for the failure are clearly revealed.

During the first $10 \mathrm{~s}$ of the experiment, only sheath solution is spun and we initially see an excellent Taylor cone in Figure 7 a. However, even without any core being injected, we see that THF as the sole solvent is not ideal: some $2 \mathrm{~s}$ into the movie, the tip of the Taylor cone starts elongating (b), clearly demonstrating that it is no longer in a fully liquid state. The volatile THF evaporates too quickly, and probably the consequent cooling of the Taylor cone also induces condensation of water, ${ }^{67}$ which dissolves in THF but is a nonsolvent for SBS. The result is gelation of the Taylor cone toward its apex, which, in panel (c), even gives it a clearly asymmetric distortion.

Because of the gelation, the spinneret is wiped clean (seen in Supporting Information Movie S4) and directly afterward (d) we start the injection of the LC core, easily recognized inside the Taylor cone by its turbid character. This initially stops the ejection of the jet and the compound Taylor cone grows in size (e) until the sheath breaks, without breaking off the core (f). Directly afterward, at $10.9 \mathrm{~s}$ into the movie (Figure $7 \mathrm{~g}$ ), a good core-sheath Taylor cone can be seen, with the core extending continuously from the spinneret orifice to the Taylor cone apex, from which a compound jet is ejected. However, already in panel $(\mathrm{g})$ one can notice a horizontal boundary of the sheath just below the spinneret orifice, and in panel (h), we clearly see that the Taylor cone curvature is different above and below this boundary. This suggests that the sheath solution starts to gel below the boundary, allowing the Taylor cone to extend more vertically here than above the boundary. Importantly, this boundary was never seen prior to injecting the LC (see panels 
$\mathrm{a}-\mathrm{c})$; hence, it is the core acting as coagulation bath that is causing this deterioration of the Taylor cone.

In panel (h), we can even start distinguishing a second boundary further down, which becomes very clear in panel (i). Below the second boundary, the Taylor cone loses its cylindrical symmetry and it distorts leftward in the image. The distortion increases in size over the next few seconds $(j)$ until that in panel $(\mathrm{k})$ is so strong that reliable spinning from the Taylor cone is no longer possible. We wipe the spinneret clean just after (k), but it takes less than $4 \mathrm{~s}$ until the Taylor cone again deforms so strongly that spinning is stopped; see Supporting Information Movie S4. Although the rapid evaporation of the volatile THF already causes problems for the single-phase spinning (this is probably why Fong and Reneker $^{73}$ and Park et al. ${ }^{74}$ used THF-DMF solvent mixtures for spinning), we see that the situation is dramatically worsened by the introduction of the core liquid that is fully miscible, without any miscibility gap, with the sheath solvent.

The elimination of core-sheath interface in the Taylor cone indeed has the advantage that the core is drawn very well into the jet, even when the Taylor cone is as distorted as in Figure $7 \mathrm{k}$. But this is of little use to us: first, because it is only a matter of seconds until spinning is stopped due to Taylor cone gelation, and, second, because the core-sheath structure is lost in the jet. This is seen in panels $(1)-(s)$, showing samples that have been collected on glass slides at different distances below the spinneret. A slide held at only $3 \mathrm{~cm}$ below the spinneret shows continuous ribbons, but they are very broad as no significant jet stretching could take place and much liquid still remains in the fiber; see Figure $71-\mathrm{m}$. Importantly, there is no trace of a core-sheath structure, and the image between crossed polarizers $(\mathrm{m})$ reveals that there is no liquid crystalline behavior at any point in the ribbon.

Moving further down to $4 \mathrm{~cm}$ below the spinneret $(\mathrm{n}-\mathrm{o})$, the ribbon has strongly undulated edges, indicating that the Rayleigh-Plateau instability is about to break it into droplets. Indeed, several droplets surround the broad ribbon that runs across the image from left to right. As before, there is neither any sign of core-sheath structure nor of liquid crystalline behavior. At a $6 \mathrm{~cm}$ distance $(\mathrm{p}-\mathrm{q})$, the instability has entirely broken up the jet and we see only large droplets, and at $7 \mathrm{~cm}$ $(\mathrm{r}-\mathrm{s})$, we see some smaller droplets and some larger regions where nearby droplets have apparently merged with-as usual-no sign of core-sheath structure or of liquid crystalline behavior. Note that much of the volatile THF should have evaporated at this point, and, in experiments without an LC core, we have indeed frequently succeeded in spinning SBS fibers in this way. However, with an LC core, its complete miscibility with the sheath solution and its non-solid state mean that the sample remains liquid, removing any trace of fibers at this stage. While the LC is not a good solvent for SBS, it is compatible enough to act as a plasticizer.

Comparison with Earlier Studies. We end by briefly revisiting some of the previously published papers discussed in the beginning in light of the new knowledge brought about by experiments. Considering first the early papers with miscible solvents by Sun et al. ${ }^{14}$ and Yu et al., ${ }^{15}$ we note that both deal with polymer solutions as cores, with the exception of one experiment by Sun et al. where the core was a THF solution of palladium(II) acetate. The entangled nature of polymer solutions and the general difficulty to mix two different polymers makes it credible that no significant mixing between core and sheath polymers takes place during the residence time in the Taylor cone when core and sheath are both polymeric. We also note that the solvents of the core and sheath solutions can be grouped into three categories: (i) identical solvents or solvent mixtures (all cases in ref 15 belong to this category), (ii) immiscible solvents (e.g., water and chloroform) but with the addition of a co-solvent that is miscible with both other solvents (in the same example ethanol in water), and (iii) different miscible solvents, but neither is a nonsolvent for any polymer (e.g., a PLA-dichloromethane sheath solution was used with the palladium acetate-THF core solution in one of the experiments of Sun et al., ${ }^{14}$ but PLA is soluble also in THF). Cases (i) and (iii) obviously give no relevant $\gamma_{c s}$, whereas case (ii) has $\gamma_{\text {cs }}$ greatly reduced by the presence of the co-solvent soluble in both phases.

We can thus conclude that these situations all avoid phase separation and Rayleigh-Plateau instabilities by keeping $\gamma_{\mathrm{cs}}$ very low; gelation is avoided by not using any nonsolvents for the polymers used; and loss of core-sheath structure is avoided by ensuring that the time from core and sheath meeting until the fiber sheath solidifies is kept much shorter than the characteristic mixing time of core and sheath solutions. Also the fabrication of hollow tubes and rods in tubes by Zussman et al. ${ }^{61}$ used polymeric core as well as sheath in identical solvents (DMF), but here also acetone was added as a co-solvent for the poly(methyl methacrylate) (PMMA) core solution. This is interesting since acetone is a nonsolvent for the PAN in the sheath; hence, the core acted as an internal coagulation bath, speeding up the solidification of the sheath. Obviously, some fine tuning is required to prevent this coagulation from starting prematurely in the Taylor cone; this may be why $40 \%$ DMF was included in the PMMA core solution.

In this context, a most interesting study is that by Luo and Edirisinghe of nonpolymeric core liquids, including water and glycerol, stabilizing electrospinning of polymer solutions as sheaths, when the same polymer solutions without a core only electrospray. ${ }^{77}$ They conducted a thorough study of miscibility of the components, finding that high $\gamma_{\text {cs }}$ can lead to welldefined fibers. While they also pointed out initially that a high $\gamma_{\text {cs }}$ promotes the Rayleigh-Plateau instability, they drew the conclusion that the fiber formation was supported by the high $\gamma_{\mathrm{cs}}$, although the mechanism for this was not clear. In light of our results and those of Zussman et al., ${ }^{61}$ we believe that the main reason for the transition from electrospray to electrospinning when using water as core liquid is that it acted as an internal coagulation bath, since the sheath solvent was miscible with water. This was not the case when using glycerol as core, but here the much greater viscosity, 3 orders of magnitude greater than that of water, is likely to be important.

The encapsulation of an industrial oil (Elf SAE-15W50) as a core inside DMF-dissolved PVP by Diaz et al. ${ }^{63}$ and Díaz Gómez et al., ${ }^{62}$ as well as inside water-dissolved PEO by Díaz Gómez et al., ${ }^{62}$ is interesting, as Díaz et al. point out that low $\gamma_{\text {cs }}$ is required, ${ }^{63}$ yet the oil-water interface when using aqueous PEO as sheath should have quite significant $\gamma_{\mathrm{cs}}$. However, the industrial oil actually contains surfactants; ${ }^{63}$ hence, this is an example where surfactant addition is a viable means of reducing $\gamma_{\mathrm{cs}}$. This approach is unfortunately not straightforward to use when working with LC cores because surfactants can strongly impact the LC alignment and even bring in emulsified water, with strong impact on the LC phase behavior. ${ }^{27}$ Despite the low $\gamma_{\mathrm{cs}}$ (values on the order of $1 \mathrm{mN} /$ $\mathrm{m}$ were mentioned), the produced fibers were strongly beaded, 
but this may also be due to a mismatch in elongational viscosities between core and sheath.

The approach to use identical solvents in sheath and core to minimize $\gamma_{c s}$ was used also by $\mathrm{He}$ et al. ${ }^{44}$ (PLA in hexafluoroisopropanol, HFIP, as sheath and the drug TCH in HFIP as core), but here the nonpolymeric nature of TCH could be expected to make retained core-sheath structure more difficult. The authors used a very high electric field (4 $\mathrm{kV} / \mathrm{cm}$ ), kept the core flow rate low, and let the inner needle protrude beyond the end of the outer needle in the coaxial spinneret. These are all features that minimize the residence time of core and sheath in the Taylor cone, which probably was kept small by the high electric field (no information about Taylor cone was provided). While López-Rubio et al. also used the same core and sheath solvent-water-in their study of bacterial inclusions in PVA sheath fibers, this fact was not discussed explicitly. ${ }^{58}$ However, since they obtained similar results using single-phase as with coaxial electrospinning, it is not obvious that a distinct core-sheath structure prevailed. In both cases, beaded fibers with bacteria contained in the beads were observed, but, given the size of the bacteria, this structure may have been driven by the bacterial cargo rather than by the coaxial spinning approach. Several studies with miscible or partially miscible cores and sheaths have made no detailed comments on the problems related to core-sheath stability, $^{30,34,37,41,43,45,46,49,53}$ but we note that several fall into category (ii) or (iii) above, and it is not unlikely that the particular combinations were found empirically by trial and error.

Returning to the paper by $\mathrm{Li}$ and $\mathrm{Xia}^{16}$ finally, which strongly promoted immiscible core and sheath, we note that the mineral oil used as core is actually not immiscible with the ethanol used as sheath solvent. The Food and Agriculture Organization of the United Nations states that mineral oil is "sparingly soluble in ethanol", ${ }^{78}$ which means that this combination of core and sheath is ideal, as they are neither fully miscible nor immiscible, but have a miscibility gap, as with RO-TN 651 and ethanol studied by us here. Since the same holds for paraffin oil/wax, and also for chloroform and DMF as solvents, this explains the success of all other coaxial electrospinning papers stating a need for immiscible core and sheath, ${ }^{17-19,38,42,66}$ inspired by the original paper by $\mathrm{Li}$ and Xia. We can also conclude that these studies did, in fact, not work with immiscible, but with partially miscible liquids, but the significance of this distinction was not clear at the time. Other papers that emphasize the needs for immiscible core and sheath liquids, such as the microtube electrospinning by Dror et al. ${ }^{65}$ or the gene delivery fibers spun by Saraf et al., ${ }^{55}$ achieve success by using mixed solvents, giving a common or at least miscible component between core and sheath.

\section{CONCLUSIONS}

By comparing core-sheath electrospinning of a nonpolymeric and nonvolatile LC core in three different sheath solutions (one miscible, one immiscible, and one partially miscible with the LC), we have demonstrated that the optimum combination is partially miscible core and sheath liquids. When the two liquids have a miscibility gap in the phase diagram that does not reach all the way to either pure component, a distinct core-sheath interface can be maintained throughout the spinning process, yet the interfacial tension $\gamma_{\mathrm{cs}}$ can be kept very low, since both liquids contain the same chemical constituents, only at different compositions. This prevents phase separation in the Taylor cone, droplet formation due to a RayleighPlateau instability in the jet, as well as loss of core-sheath structure due to complete mixing. In this way, the production of fibers with continuous core-sheath morphology can be ensured, of great value when the core adds functionality to the fiber. Importantly, the core should not be spun pure, but enough of the sheath solvent should be added to reach the miscibility gap of the phase diagram: otherwise, strong Marangoni stresses and, in turn, complex flow patterns can arise in the Taylor cone as a consequence of local variations of $\gamma_{c s}$, preventing stable core-sheath spinning. While our experiments were conducted with an LC core, these conclusions are perfectly applicable to any other core-sheath combination.

\section{ASSOCIATED CONTENT}

\section{Supporting Information}

The Supporting Information is available free of charge at https://pubs.acs.org/doi/10.1021/acs.langmuir.1c01824.

Interfacial tension characterization of the spinning solutions; snapshots of the interfacial tension measurements using the pendant drop technique of RO-TN 651 to a solution of $10 \% \mathrm{w} / \mathrm{w}$ PAA in anhydrous ethanol (Figure S1); electrospinning parameters and conditions (Table S1) (PDF)

Taylor cone recorded during electrospinning RO-TN 651 as core and an aqueous solution of $11.5 \% \mathrm{w} / \mathrm{w}$ PAA as sheath (Movie S1) (MP4)

Taylor cone recorded during electrospinning RO-TN 651 as the core and a solution of $10 \% \mathrm{w} / \mathrm{w}$ PAA in anhydrous ethanol as sheath (Movie S2) (MP4)

Taylor cone recorded during electrospinning of 10\% w/ $\mathrm{w}$ anhydrous ethanol in RO-TN 651 core and a solution of $10 \% \mathrm{w} / \mathrm{w}$ PAA in anhydrous as sheath (Movie S3) (MP4)

Taylor cone during electrospinning of $10 \% \mathrm{w} / \mathrm{w}$ THF in RO-TN 651 as the core and a $10 \% \mathrm{w} / \mathrm{w}$ SBS in THF, as sheath solution (Movie S4) (MP4)

Interfacial tension measurement using the pendant drop technique with a drop of RO-TN 651 injected at $0.5 \mu \mathrm{L} /$ $\mathrm{s}$ into a bath of aqueous solution of $11.5 \% \mathrm{w} / \mathrm{w}$ PAA (Movie S5) (MP4)

Interfacial tension measurement using the pendant drop technique with a drop of RO-TN 651 injected at $0.1 \mu \mathrm{L} /$ $\mathrm{s}$ into a bath of $10 \% \mathrm{w} / \mathrm{w}$ PAA solution in anhydrous ethanol (Movie S6) (MP4)

Interfacial tension measurement using the pendant drop technique with a drop of RO-TN 651 injected at $0.05 \mu \mathrm{L} / \mathrm{s}$ into a bath of $10 \% \mathrm{w} / \mathrm{w}$ PAA solution in anhydrous ethanol (Movie S7) (MP4)

Interfacial tension measurement using the pendant drop technique with a drop of $10 \% \mathrm{w} / \mathrm{w}$ anhydrous ethanol in RO-TN 651 injected at $0.1 \mu \mathrm{L} / \mathrm{s}$ into a bath of $10 \% \mathrm{w} / \mathrm{w}$ PAA solution in anhydrous ethanol (Movie S8) (MP4)

\section{AUTHOR INFORMATION}

\section{Corresponding Author}

Jan P. F. Lagerwall - Experimental Soft Matter Physics Group, University of Luxembourg, L-1511 Luxembourg, Luxembourg; (1) orcid.org/0000-0001-9753-1147; Phone: +352 466644 6219; Email: jan.lagerwall@ lcsoftmatter.com; Fax: +352 46664436219 


\section{Authors}

Shameek Vats - Experimental Soft Matter Physics Group, University of Luxembourg, L-1511 Luxembourg, Luxembourg

Manos Anyfantakis - Experimental Soft Matter Physics Group, University of Luxembourg, L-1511 Luxembourg, Luxembourg; o orcid.org/0000-0002-4572-5641

Lawrence W. Honaker - Experimental Soft Matter Physics Group, University of Luxembourg, L-1511 Luxembourg, Luxembourg; Laboratory of Physical Chemistry and Soft Matter, Wageningen University \& Research, 6703 DE Wageningen, The Netherlands

Francesco Basoli - Department of Engineering, Università Campus Bio-Medico di Roma, 00128 Rome, Italy

Complete contact information is available at: https://pubs.acs.org/10.1021/acs.langmuir.1c01824

\section{Notes}

The authors declare no competing financial interest.

\section{ACKNOWLEDGMENTS}

Funding for this research was provided under the European Union's H2020 Programme/ERC Grant Agreement No. 648763 (consolidator project INTERACT) and by an Aide à la formation-recherche grant (LIMEFLOW, grant number 9784104) and a CORE Junior grant (COReLIGHT, grant number C18/MS/12701231) from the Luxembourg National Research Fund. The authors thank Nicolas Tournier, Dr. Hakam Agha, and Dr. Ulrich M. Siegel for assistance in constructing the experimental setup; Anna Nauclér and Edvard Nauclér for assistance in establishing the phase diagram in Figure ${ }^{4}$; Dr. Anupam Sengupta for allowing access to his laboratory; Dr. Vamseekrishna Ulaganathan for his assistance in performing interfacial tension measurements; and Dr. Anshul Sharma, Dr. Catherine G. Reyes, Dr. V.S.R. Jampani, and Katrin Schelski for fruitful discussions.

\section{ADDITIONAL NOTE}

"Some refer to this as "triaxial" spinning, but this is unfortunately an incorrect and even misleading terminology; both the spinnerets and the fibers are uniaxial, as indeed required by a coaxial geometry.

\section{REFERENCES}

(1) Formhals, A. Process and Apparatus for Preparing Artificial Threads. U.S. Patent US1975504A1934.

(2) Huang, Z.; Zhang, Y.; Kotaki, M.; Ramakrishna, S. A review on polymer nanofibers by electrospinning and their applications in nanocomposites. Compos. Sci. Technol. 2003, 63, 2223-2253.

(3) Li, D.; Xia, Y. Electrospinning of Nanofibers: Reinventing the Wheel? Adv. Mater. 2004, 16, 1151-1170.

(4) Greiner, A.; Wendorff, J. Electrospinning: A fascinating method for the preparation of ultrathin fibres. Angew. Chem., Int. Ed. 2007, 46, 5670-5703.

(5) Rutledge, G.; Fridrikh, S. Formation of fibers by electrospinning. Adv. Drug Delivery Rev. 2007, 59, 1384-1391.

(6) Reneker, D.; Yarin, A. Electrospinning jets and polymer nanofibers. Polymer 2008, 49, 2387-2425.

(7) Lu, X.; Wang, C.; Wei, Y. One-Dimensional Composite Nanomaterials: Synthesis by Electrospinning and Their Applications. Small 2009, 5, 2349-2370.

(8) Xue, J.; Wu, T.; Dai, Y.; Xia, Y. Electrospinning and Electrospun Nanofibers: Methods, Materials, and Applications. Chem. Rev. 2019, 119, 5298-5415.
(9) Yarin, A. Coaxial Electrospinning and Emulsion Electrospinning of Core-shell Fibers. Polym. Adv. Technol. 2011, 22, 310-317.

(10) Yoon, J.; Yang, H.; Lee, B.; Yu, W. Recent Progress in Coaxial Electrospinning: New Parameters, Various Structures, and Wide Applications. Adv. Mater. 2018, 30, No. e1704765.

(11) Pant, B.; Park, M.; Park, S.-J. Drug Delivery Applications of Core-Sheath Nanofibers Prepared by Coaxial Electrospinning: A Review. Pharmaceutics 2019, 11, No. 305.

(12) Han, D.; Steckl, A. J. Coaxial Electrospinning Formation of Complex Polymer Fibers and their Applications. ChemPlusChem 2019, 84, 1453-1497.

(13) Rathore, P.; Schiffman, J. Beyond the Single-Nozzle: Coaxial Electrospinning Enables Innovative Nanofiber Chemistries, Geometries, and Applications. ACS Appl. Mater. Interfaces 2021, 13, 4866.

(14) Sun, Z.; Zussman, E.; Yarin, A.; Wendorff, J.; Greiner, A. Compound core-shell polymer nanofibers by co-electrospinning. Adv. Mater. 2003, 15, 1929-1932.

(15) Yu, J. H.; Fridrikh, S. V.; Rutledge, G. C. Production of submicrometer diameter fibers by two-fluid electrospining. $A d v$. Mater. 2004, 16, 1562-1566.

(16) Li, D.; Xia, Y. Direct Fabrication of Composite and Ceramic Hollow Nanofibers By Electrospinning. Nano Lett. 2004, 4, 933-938.

(17) McCann, J.; Marquez, M.; Xia, Y. Melt coaxial electrospinning: A versatile method for the encapsulation of solid materials and fabrication of phase change nanofibers. Nano. Lett. 2006, 6, 28682872.

(18) Wang, N.; Chen, H.; Lin, L.; Zhao, Y.; Cao, X.; Song, Y.; Jiang, L. Multicomponent Phase Change Microfibers Prepared by Temperature Control Multifluidic Electrospinning. Macromol. Rapid Commun. 2010, 31, 1622-1627.

(19) Lu, Y.; Xiao, X.; Fu, J.; Huan, C.; Qi, S.; Zhan, Y.; Zhu, Y.; Xu, G. Novel smart textile with phase change materials encapsulated coresheath structure fabricated by coaxial electrospinning. Chem. Eng. J. 2019, 355, 532-539.

(20) Lagerwall, J. P. F.; McCann, J. T.; Formo, E.; Scalia, G.; Xia, Y. Coaxial Electrospinning of Microfibres With Liquid Crystal in the Core. Chem. Commun. 2008, 42, 5420-5422.

(21) Enz, E.; Baumeister, U.; Lagerwall, J. Coaxial Electrospinning of Liquid Crystal-containing Poly(vinyl Pyrrolidone) Microfibers. Beilstein J. Org. Chem. 2009, 5, No. 58.

(22) Enz, E.; Lagerwall, J. P. F. Electrospun microfibres with temperature sensitive iridescence from encapsulated cholesteric liquid crystal. J. Mater. Chem. 2010, 20, 6866-6872.

(23) Buyuktanir, E. A.; Frey, M. W.; West, J. L. Self-assembled, Optically Responsive Nematic Liquid Crystal/polymer Core-shell Fibers: Formation and Characterization. Polymer 2010, 51, 48234830.

(24) Liang, H.-L.; Enz, E.; Scalia, G.; Lagerwall, J. Liquid Crystals in Novel Geometries prepared by Microfluidics and Electrospinning. Mol. Cryst. Liq. Cryst. 2011, 549, 69-77.

(25) Kim, D. K.; Hwang, M.; Lagerwall, J. P. F. Liquid crystalfunctionalization of electrospun polymer fibers. J. Polym. Sci., Part B: Polym. Phys. 2013, 51, 855-867.

(26) Scalia, G.; Enz, E.; Calò, O.; Kim, D. K.; Hwang, M.; Lee, J. H.; Lagerwall, J. P. F. Morphology and Core Continuity of Liquid-crystalfunctionalized, Coaxially Electrospun Fiber Mats Tuned Via the Polymer Sheath Solution. Macromol. Mater. Eng. 2013, 298, 583-589.

(27) Kye, Y.; Kim, C.; Lagerwall, J. Multifunctional responsive fibers produced by dual liquid crystal core electrospinning. J. Mater. Chem. C 2015, 3, 8979-8985.

(28) Wang, J.; Jákli, A.; West, J. Morphology Tuning of Electrospun Liquid Crystal/Polymer Fibers. ChemPhysChem 2016, 17, 30803085.

(29) Reyes, C. G.; Sharma, A.; Lagerwall, J. P. Non-electronic gas sensors from electrospun mats of liquid crystal core fibers for detecting volatile organic compounds at room temperature. Liq. Cryst. 2016, 43, 1986-2001. 
(30) Lin, J.; Chen, C.; Chen, L.; Chuang, Y.; Huang, S.; Lee, C.-R. Morphological appearances and photo-controllable coloration of dyedoped cholesteric liquid crystal/polymer coaxial microfibers fabricated by coaxial electrospinning technique. Opt. Express 2016, 24, $3112-3126$

(31) Bertocchi, M. J.; Ratchford, D. C.; Casalini, R.; Wynne, J. H.; Lundin, J. G. Electrospun Polymer Fibers Containing a Liquid Crystal Core: Insights into Semiflexible Confinement. J. Phys. Chem. C 2018, 122, 16964-16973.

(32) Wang, J.; Jákli, A.; West, J. L. Liquid crystal/polymer fiber mats as sensitive chemical sensors. J. Mol. Liq. 2018, 267, 490-495.

(33) Dicker, K. T.; Ratchford, D.; Casalini, R.; Thum, M. D.; Wynne, J. H.; Lundin, J. G. Surfactant Modulated Phase Transitions of Liquid Crystals Confined in Electrospun Coaxial Fibers. Langmuir 2020, 36, 7916-7924.

(34) Thum, M. D.; Ratchford, D. C.; Casalini, R.; Wynne, J. H.; Lundin, J. G. Azobenzene-Doped Liquid Crystals in Electrospun Nanofibrous Mats for Photochemical Phase Control. ACS Appl. Nano Mater. 2021, 4, 297-304.

(35) Mamuk, A. E.; Koçak, Ç.; Demirci Dönmez, ÇE. Production and characterization of liquid crystal/polyacrylonitrile nano-fibers by electrospinning method. Colloid Polym. Sci. 2021, 299, 1209-1221.

(36) Jasiurkowska-Delaporte, M.; Juszyńska-Gałązka, E.; Sas, W.; Zieliński, P. M.; Baranowska-Korczyc, A. Soft versus hard confinement effects on the phase transitions, and intra- and inter-molecular dynamics of 6BT liquid crystal constrained in electrospun polymer fibers and in nanopores. J. Mol. Liq. 2021, 331, No. 115817.

(37) Bertocchi, M. J.; Vang, P.; Balow, R. B.; Wynne, J. H.; Lundin, J. G. Enhanced Mechanical Damping in Electrospun Polymer Fibers with Liquid Cores: Applications to Sound Damping. ACS Appl. Polym. Mater. 2019, 1, 2068-2076.

(38) Chen, H.; Wang, N.; Di, J.; Zhao, Y.; Song, Y.; Jiang, L. Nanowire-in-microtube Structured Core/shell Fibers Via Multifluidic Coaxial Electrospinning. Langmuir 2010, 26, 11291-11296.

(39) Liu, W.; Ni, C.; Chase, D. B.; Rabolt, J. F. Preparation of Multilayer Biodegradable Nanofibers by Triaxial Electrospinning. ACS Macro Lett. 2013, 2, 466-468.

(40) Han, D.; Steckl, A. Triaxial electrospun nanofiber membranes for controlled dual release of functional molecules. ACS Appl. Mater. Interfaces 2013, 5, 8241-8245.

(41) Jiang, S.; Duan, G.; Zussman, E.; Greiner, A.; Agarwal, S. Highly flexible and tough concentric triaxial polystyrene fibers. ACS Appl. Mater. Interfaces 2014, 6, 5918-5923.

(42) Zhao, Y.; Cao, X.; Jiang, L. Bio-mimic Multichannel Microtubes By a Facile Method. J. Am. Chem. Soc. 2007, 129, 764-765.

(43) He, C.-L.; Huang, Z.-M.; Han, X.-J.; Liu, L.; Zhang, H.-S.; Chen, L.-S. Coaxial Electrospun Poly(L-Lactic Acid) Ultrafine Fibers for Sustained Drug Delivery. J. Macromol. Sci., Part B 2006, 45, 515524.

(44) He, C.-L.; Huang, Z.-M.; Han, X.-J. Fabrication of drug-loaded electrospun aligned fibrous threads for suture applications. J. Biomed. Mater. Res., Part A 2009, 89, 80-95.

(45) Wang, C.; Yan, K.-W.; Lin, Y.-D.; Hsieh, P. C. H. Biodegradable Core/Shell Fibers by Coaxial Electrospinning: Processing, Fiber Characterization, and Its Application in Sustained Drug Release. Macromolecules 2010, 43, 6389-6397.

(46) Jiang, Y.; Mo, H.; Yu, D. Electrospun drug-loaded core-sheath $\mathrm{PVP} /$ zein nanofibers for biphasic drug release. Int. J. Pharm. 2012, 438, 232-239.

(47) Yu, D.; Yu, J.; Chen, L.; Williams, G.; Wang, X. Modified coaxial electrospinning for the preparation of high-quality ketoprofenloaded cellulose acetate nanofibers. Carbohydr. Polym. 2012, 90, $1016-1023$

(48) Zamani, M.; Prabhakaran, M.; Ramakrishna, S. Advances in drug delivery via electrospun and electrosprayed nanomaterials. Int. J. Nanomed. 2013, 8, 2997-3017.

(49) Yu, D.; Wang, X.; Li, X.; Chian, W.; Li, Y.; Liao, Y. Electrospun biphasic drug release polyvinylpyrrolidone/ethyl cellulose core/sheath nanofibers. Acta Biomater. 2013, 9, 5665-5672.
(50) Chou, S.; Carson, D.; Woodrow, K. Current strategies for sustaining drug release from electrospun nanofibers. J. Controlled Release 2015, 220, 584-591.

(51) Wang, Q.; Yu, D.; Zhang, L.; Liu, X.; Deng, Y.; Zhao, M. Electrospun hypromellose-based hydrophilic composites for rapid dissolution of poorly water-soluble drug. Carbohydr. Polym. 2017, 174, 617-625.

(52) Khoshnevisan, K.; Maleki, H.; Samadian, H.; Shahsavari, S.; Sarrafzadeh, M.; Larijani, B.; Dorkoosh, F.; Haghpanah, V.; Khorramizadeh, M. Cellulose acetate electrospun nanofibers for drug delivery systems: Applications and recent advances. Carbohydr. Polym. 2018, 198, 131-141.

(53) Sahoo, S.; Ang, L.; Goh, J.; Toh, S. Growth factor delivery through electrospun nanofibers in scaffolds for tissue engineering applications. J. Biomed. Mater. Res., Part A 2010, 93, 1539-1550.

(54) Ji, W.; Sun, Y.; Yang, F.; van den Beucken, J.; Fan, M.; Chen, Z.; Jansen, J. Bioactive electrospun scaffolds delivering growth factors and genes for tissue engineering applications. Pharm. Res. 2011, 28, $1259-1272$.

(55) Saraf, A.; Baggett, L.; Raphael, R.; Kasper, F.; Mikos, A. Regulated non-viral gene delivery from coaxial electrospun fiber mesh scaffolds. J. Controlled Release 2010, 143, 95-103.

(56) Townsend-Nicholson, A.; Jayasinghe, S. N. Cell Electrospinning: a Unique Biotechnique for Encapsulating Living Organisms for Generating Active Biological Microthreads/Scaffolds. Biomacromolecules 2006, 7, 3364-3369.

(57) Klein, S.; Kuhn, J.; Avrahami, R.; Tarre, S.; Beliavski, M.; Green, M.; Zussman, E. Encapsulation of Bacterial Cells in Electrospun Microtubes. Biomacromolecules 2009, 10, 1751-1756.

(58) López-Rubio, A.; Sanchez, E.; Sanz, Y.; Lagaron, J. Encapsulation of living bifidobacteria in ultrathin $\mathrm{PVOH}$ electrospun fibers. Biomacromolecules 2009, 10, 2823-2829.

(59) Pschyklenk, L.; Wagner, T.; Lorenz, A.; Kaul, P. Optical Gas Sensing with Encapsulated Chiral-Nematic Liquid Crystals. ACS Appl. Polym. Mater. 2020, 2, 1925-1932.

(60) Chiu, Y.; Weng, C.; Tseng, H.; Hsu, H.; Chen, J. Snake Tracks in Polymer Land: Wavy Polymer Structures via Selective Solvent Vapor Annealing. Langmuir 2020, 36, 9780-9785.

(61) Zussman, E.; Yarin, A.; Bazilevsky, A.; Avrahami, R.; Feldman, M. Electrospun Polyaniline/Poly(methyl methacrylate)-Derived Turbostratic Carbon Micro-/Nanotubes. Adv. Mater. 2006, 18, $348-353$.

(62) Díaz Gómez, J. E.; Marín, Á. G.; Marquez, M.; Barrero, A.; Loscertales, I. G. Encapsulation and suspension of hydrophobic liquids via electro-hydrodynamics. Biotechnol. J. 2006, 1, 963-968.

(63) Díaz, J. E.; Barrero, A.; Márquez, M.; Loscertales, I. G. Controlled encapsulation of hydrophobic liquids in hydrophilic polymer nanofibers by co-electrospinning. Adv. Funct. Mater. 2006, $16,2110-2116$.

(64) Loscertales, I.; Barrero, A.; Guerrero, I.; Cortijo, R.; Marquez, M.; Ganan-Calvo, A. Micro/nano encapsulation via electrified coaxial liquid jets. Science 2002, 295, 1695-1698.

(65) Dror, Y.; Salalha, W.; Avrahami, R.; Zussman, E.; Yarin, A.; Dersch, R.; Greiner, A.; Wendorff, J. One-step production of polymeric microtubes by co-electrospinning. Small 2007, 3, 10641073.

(66) Chan, K.; Kotaki, M. Fabrication and morphology control of poly (methyl methacrylate) hollow structures via coaxial electrospinning. J. Appl. Polym. Sci. 2008, 111, 408-416.

(67) Reyes, C. G.; Lagerwall, J. P. F. Disruption of Electrospinning due to Water Condensation into the Taylor Cone. ACS Appl. Mater. Interfaces 2020, 12, 26566-26576.

(68) Vazquez, G.; Alvarez, E.; Navaza, J. M. Surface tension of alcohol + water from 20 to $50^{\circ} \mathrm{C}$. J. Chem. Eng. Data 1995, 40, 611614.

(69) Kim, D. K.; Lagerwall, J. P. F. Influence of Wetting on Morphology and Core Content in Electrospun Core-Sheath Fibers. ACS Appl. Mater. Interfaces 2014, 6, 16441-16447. 
(70) Reyes, C. G.; Baller, J.; Araki, T.; Lagerwall, J. P. F. Isotropicisotropic phase separation and spinodal decomposition in liquid crystal-solvent mixtures. Soft Matter 2019, 6044-6054.

(71) Scriven, L.; Sternling, C. The Marangoni effects. Nature 1960, 187, 186-188.

(72) Fanton, X.; Cazabat, A. Spreading and instabilities induced by a solutal Marangoni effect. Langmuir 1998, 14, 2554-2561.

(73) Fong, H.; Reneker, D. H. Elastomeric nanofibers of styrenebutadiene-styrene triblock copolymer. J. Polym. Sci., Part B: Polym. Phys. 1999, 37, 3488-3493.

(74) Park, M.; Im, J.; Shin, M.; Min, Y.; Park, J.; Cho, H.; Park, S.; Shim, M.; Jeon, S.; Chung, D.; Bae, J.; Park, J.; Jeong, U.; Kim, K. Highly stretchable electric circuits from a composite material of silver nanoparticles and elastomeric fibres. Nat. Nanotechnol. 2012, 7, 803809.

(75) Lin, C.; Jiang, D.; Kuo, C.; Cho, C.; Tsai, Y.; Satoh, T.; Su, C. Water-Resistant Efficient Stretchable Perovskite-Embedded Fiber Membranes for Light-Emitting Diodes. ACS Appl. Mater. Interfaces 2018, 10, 2210-2215.

(76) Marriam, I.; Wang, X.; Mebyetekerwa, M.; Chen, G.; Zabihi, F.; Pionteck, J.; Peng, S.; Ramakrishna, S.; Yang, S.; Zhu, M. A bottom-up approach to design wearable and stretchable smart fibers with organic vapor sensing behaviors and energy storage properties. J. Mater. Chem. A 2018, 6, 3633-13643.

(77) Luo, C.; Edirisinghe, M. Core-liquid-induced transition from coaxial electrospray toelectrospinning of low-viscosity poly (lactideco-glycolide) sheath solution. Macromolecules 2014, 47, 7930-7938.

(78) The Food and Agriculture Organization of the United Nations. Mineral Oil (Medium Viscosity). In FAO JECFA Monographs; FAO, 2013; Vol. 14. 


\title{
Supplementary Information for "Stable electrospinning of core-functionalized coaxial fibers enabled by the minimum-energy interface given by partial core-sheath miscibility"
}

\author{
Shameek Vats ${ }^{a}$, Manos Anyfantakis ${ }^{a}$, Lawrence W. Honaker ${ }^{a, c}$, Francesco Basoli $^{b}$, and \\ Jan P.F. Lagerwall ${ }^{a}$ \\ ${ }^{a}$ Experimental Soft Matter Physics Group, University of Luxembourg, L-1511 Luxembourg, \\ Luxembourg \\ ${ }^{b}$ Department of Engineering, Università Campus Bio-Medico di Roma, 00128 Rome, Italy \\ ${ }^{c}$ Laboratory of Physical Chemistry and Soft Matter, Wageningen University 85 Research, \\ 6703 DE Wageningen, The Netherlands
}

\section{Discussion about interfacial tension experiments}

\section{RO-TN 651-(PAA/water) system}

To measure the interfacial tension of the RO-TN 651-(PAA/water) system, we used a stainless steel needle to make a pendant drop of the LC phase inside a bath of a PAA/water solution $(11.5 \% \mathrm{w} / \mathrm{w})$. The experiments were performed at $20 \circ \mathrm{C}$. From three independent measurements (i.e., using three different drops), we measured an average value of $9.13 \pm 0.30 \mathrm{mN} / \mathrm{m}$ (the error is the standard deviation).

The SI Movie 5 shows a representative example of such a drop. Even at very large volumes (up to $35 \mu \mathrm{L}$; beyond this volume the drop profile exceeds the imaging window), the drop is neither strongly deformed by gravity, nor can it be detached by the needle (unless we perturb it mechanically). This is attributed to the very low density difference between RO-TN 651 $\left(1.0469 \mathrm{~g} / \mathrm{cm}^{3}\right)$ and the PAA/water solution $\left(1.0358 \mathrm{~g} / \mathrm{cm}^{3}\right)$, respectively, which minimizes the effect of gravity on the drop. After we measured the interfacial tension in the stationary drop, we performed a few cycles of first pushing the needle against the drop, and then pulling it away from the drop. It can be clearly seen in SI Movie 5 that the drop responds to these 
perturbations by quickly relaxing into its equilibrium quasi-spherical shape, indicating that $9 \mathrm{mN} / \mathrm{m}$ is indeed a significant (albeit generally low) interfacial tension.

\section{RO-TN 651-(PAA/ethanol) system}

To perform interfacial tension experiments with the RO-TN 651-(PAA/ethanol) system, we used a stainless steel needle to create a pendant drop of the LC phase inside a bath of a PAA/ethanol solution $(10.0 \% \mathrm{w} / \mathrm{w})$. The experiments were performed at $21 \circ \mathrm{C}$. SI Movie 7 shows the production of LC drops inside the polymer solution bath. Even by employing a very low flow rate $(0.05 \mu \mathrm{L} / \mathrm{s})$, a stable drop cannot be formed. Instead, a series of small drops connected by a transient LC jet are produced. The LC jet seems to progressively dissolve in the PAA/ethanol solution, leading to individual RO-TN 651 drops that move towards the bottom of the sample cell due to gravity. Interestingly, drops that come into contact do not coalesce (within the time of the experiment), indicating some extent of stabilization, presumably by PAA chains adsorbing onto the LC-polymer solution interface. In addition, the ejected drops seem to slightly increase in size as they sediment. This suggests that swelling of the LC drop due to ethanol diffusing into its interior might take place. This is further supported by the observation that the drop-solution interface becomes less well-defined, macroscopically, as seen from the decreasing sharpness of the fluid boundary. This qualitative picture is in accordance with the phase behavior of the ROTN 651-(PAA/ethanol) system (Fig.4) that shows that the two phases are fully miscible at very low ethanol concentrations; the pendant drop experiment described here is analogous to this case, considering that we have droplets with volumes on the order of microliters inside a bath with volume on the order of a few milliliters.

Even if we stop dosing the LC after a first drop is formed, this drop does not remain stable; the LC keeps flowing, even though there is no external pressure imposed on the syringe. The inability to make a stationary drop prevents us from measuring the equilibrium interfacial tension of the RO-TN 651-(PAA/ethanol) system. SI Movie 6 shows the early 
stage of the formation of a LC drop, under the optimum conditions we identified (target drop volume $1 \mu \mathrm{L}$, flow rate $0.1 \mu \mathrm{L} / \mathrm{s}$ ); higher flow rates led to a pronounced ejection of LC drops connected by a LC jet, whereas lower flow rates resulted in the case where the LC drop is not in full contact with the whole orifice of the needle. Under these experimental conditions, a well-defined drop can be formed, which is followed by the formation of a second drop that is however highly non-spherical (due to the broken jet created in its front). Fig. S1 shows snapshots from the formation of the first drop, with $t=0 \mathrm{~s}$ corresponding to the time where the first image of the shown series was recorded. Clearly, the drop shape analysis cannot give reliable interfacial tension values because the pendant drop is not stationary (pendant drop tensiometry requires a stationary drop, the shape of which is dictated by the balance of surface tension and gravity). Despite this fact, we show in Fig. S1 fits of the drop profile; as can be seen, we can fit reasonably well the shape of the pendant drop recorded in the first two images (i.e., 0 and $0.46 \mathrm{~s}$ ), while the fitting becomes worse for longer times. Although the calculated, transient interfacial tension values (about $3 \mathrm{mN} / \mathrm{m}$ ) cannot be trusted absolutely, we get an idea about their order of magnitude; they are very low. This picture of a diminishing interfacial tension between the RO-TN 651 and the PAA/ethanol phases is consistent with what would one expect for an interface formed by two phases consisting of the same chemical constituents, but at different compositions.

\section{(RO-TN 651/ethanol)-(PAA/ethanol) system}

To conduct interfacial tension experiments with the (RO-TN 651/ethanol)-(PAA/ethanol) system, we used a stainless steel needle to make a pendant drop of the ethanol-containing LC phase inside a bath of a PAA/ethanol solution $(10.0 \% \mathrm{w} / \mathrm{w})$. The experiments were performed at $21 \circ \mathrm{C}$. A few problems that prevent the formation of a well-defined, stable pendant drop of LC are shown in SI Movie 8. First, as the LC phase is pushed through the needle, a soft, solid-like phase of irregular shape emerges first. We believe this is highly concentrated (and perhaps gelled) PAA/ethanol solution that was previously left at the 
opening of the needle. This can be due to drying of a portion of this solution that was left at the tip of the needle while it is removed from the solution bath in order to be cleaned. Without this cleaning cycle, we are not able to form a pendant drop at all. Furthermore, as more LC is flushed through the needle, an irregularly shaped LC drop emerges which surrounds the solid-like polymer solution phase. At longer times, and with more LC emerging from the orifice, more drops are formed that are initially separated from the first LC drop before they eventually merge to form a large, irregularly-shaped LC drop. The difficulty in merging, in conjunction with the irregular shape of the LC-polymer solution interface (which is nevertheless well-defined), suggests that the LC phase is stabilized by the polymer solution, presumably by PAA chains adsorbing onto the fluid interface. While interesting, these observations clearly show that pendant drop tensiometry is not an appropriate method to measure the interfacial tension of this highly complex system.

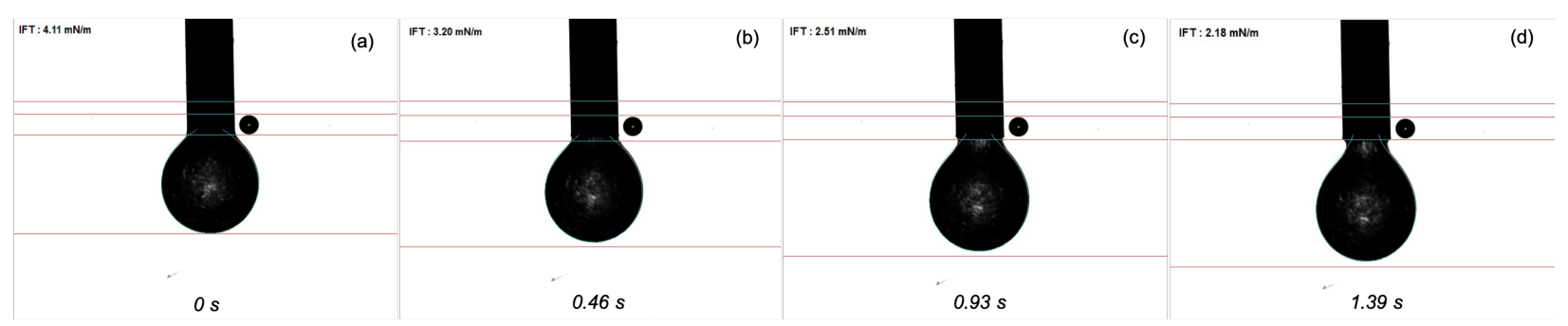

Figure S1: Snapshots of the interfacial tension measurements using pendant drop technique of ROTN 651 to a solution of $10 \% \mathrm{w} / \mathrm{w}$ PAA in anhydrous ethanol. These still frames are extracted from SI movie 6, the outer diameter of the stainless steel needle used for the measurements is $0.51 \mathrm{~mm}$.

\section{Electrospinning Parameters}

Table S1 gives the electrospinning parameters used in the experiments. 
Table S1: Electrospinning parameters and conditions.

\begin{tabular}{|c|c|c|c|c|c|c|c|}
\hline & & $\begin{array}{c}\text { Relative } \\
\text { Humidity(\%) }\end{array}$ & $\begin{array}{c}\text { Temperature } \\
(\mathrm{o} \mathrm{C})\end{array}$ & $\begin{array}{c}\text { Distance } \\
(\mathrm{cm})\end{array}$ & $\begin{array}{c}\text { Voltage } \\
(\mathrm{kV})\end{array}$ & \multicolumn{2}{|c|}{$\begin{array}{c}\text { Flow rate } \\
(\mathrm{ml} / \mathrm{h})\end{array}$} \\
\hline Sheath & Core & & & & & Sheath & Core \\
\hline $\begin{array}{c}11.5 \% \text { w/w PAA in } \\
\text { water }\end{array}$ & RO-TN 651 & 25 & 22.6 & 15 & 9.8 & 5.1 & 0.24 \\
\hline $\begin{array}{c}10 \% \text { w/w PAA in } \\
\text { anhydrous ethanol }\end{array}$ & RO-TN 651 & 24 & 22.1 & 15 & 6.9 & 19.68 & 0.24 \\
\hline $\begin{array}{c}10 \% \text { w/w PAA in } \\
\text { anhydrous ethanol }\end{array}$ & $\begin{array}{c}10 \% \text { w/w anhydrous } \\
\text { ethanol in RO-TN 651 }\end{array}$ & 36 & 22.6 & 15 & 5.4 & 15.3 & 0.42 \\
\hline
\end{tabular}

\section{SI Videos}

SI Movie 1: Movie of the Taylor cone recorded during electrospinning RO-TN 651 as core and an aqueous solution of $11.5 \% \mathrm{w} / \mathrm{w}$ PAA as sheath. The outer diameter of the spinneret needle is $1.7 \mathrm{~mm}$ and the electrospinning parameters and conditions are listed in the Table S1. This movie corresponds to Figure 3.

SI Movie 2: Movie of the Taylor cone recorded during electrospinning RO-TN 651 as the core and a solution of $10 \% \mathrm{w} / \mathrm{w}$ PAA in anhydrous ethanol as sheath. The outer diameter of the spinneret needle is $1.7 \mathrm{~mm}$ and the electrospinning parameters and conditions are listed in the Table S1. This movie corresponds to Figure5.

SI Movie 3: Movie of the Taylor cone recorded during electrospinning of $10 \% \mathrm{w} / \mathrm{w}$ anhydrous ethanol in RO-TN 651 core and a solution of 10\% w/w PAA in anhydrous as sheath. The outer diameter of the spinneret needle is $1.7 \mathrm{~mm}$ and the electrospinning parameters and conditions are listed in the Table S1. This movie corresponds to Figure 6.

SI Movie 4: Movie of the Taylor cone during electrospinning of $10 \% \mathrm{w} / \mathrm{w}$ THF in ROTN 651 as the core and a 10\% w/w SBS in THF, as sheath solution. The outer diameter of the spinneret needle is $1.7 \mathrm{~mm}$. This movie corresponds to the Figure 7.

SI Movie 5: Interfacial tension measurement using pendant drop technique with a drop of RO-TN 651 immersed in a bath of aqueous solution of $11.5 \% \mathrm{w} / \mathrm{w}$ PAA. The flow rate of the pendant drop is $0.5 \mu \mathrm{L} / \mathrm{s}$ and the diameter of the stainless steel needle used is $0.51 \mathrm{~mm}$. 
SI Movie 6: Interfacial tension measurement using pendant drop technique with a drop of RO-TN 651 immersed in a bath of 10\%w/w PAA solution in anhydrous ethanol. The flow rate of the pendant drop is $0.1 \mu \mathrm{L} / \mathrm{s}$ and the diameter of the stainless steel needle used is $0.51 \mathrm{~mm}$.

SI Movie 7: Interfacial tension measurement using pendant drop technique with a drop of RO-TN 651 immersed in a bath of $10 \% \mathrm{w} / \mathrm{w}$ PAA solution in anhydrous ethanol. The flow rate of the pendant drop is $0.05 \mu \mathrm{L} / \mathrm{s}$ and the diameter of the stainless steel needle used is $0.51 \mathrm{~mm}$.

SI Movie 8: Interfacial tension measurement using pendant drop technique with a drop of $10 \% \mathrm{w} / \mathrm{w}$ anhydrous ethanol in RO-TN 651 immersed in a bath of 10\%w/w PAA solution in anhydrous ethanol. The flow rate of the pendant drop is $0.1 \mu \mathrm{L} / \mathrm{s}$ and the diameter of the Teflon needle used is $0.8 \mathrm{~mm}$. 



\title{
Electrospinning Ethanol-Water Solutions of Poly(Acrylic Acid): Nonlinear Viscosity Variations and Dynamic Taylor Cone Behavior
}

\author{
Shameek Vats, Lawrence W. Honaker, Margaret W. Frey, Francesco Basoli, \\ and Jan P.F. Lagerwall**
}

Electrospinning of polymer solutions is a multifaceted process that depends on the careful balancing of many parameters to achieve a desired outcome, in many cases including mixtures of multiple solvents. A systematic study of how the solution viscosity $\eta$-a good probe of solvent-polymer interactions - and the electrospinnability change when poly(acrylic acid) (PAA) is dissolved in ethanol-water mixtures at varying mixing ratio is carried out. A pronounced maximum is found in $\eta$ at a water-to-ethanol molar ratio of about 2:1, where the solvent mixture deviates maximally from ideal mixing behavior and partial deprotonation of carboxyl groups by water coincides synergistically with dissolution of the uncharged protonated PAA fraction by ethanol. The PAA concentration is tuned as a function of water-ethanol ratio to obtain a common value of $\eta$ for all solvent mixtures that is suitable for electrospinning. For high PAA content, the Taylor cone grows in volume over time despite minimum solution flow rate, even experiencing surface gelation for ethanol-rich solutions. This is attributed to the hygroscopic nature of PAA, drawing excess water into the Taylor cone from the air during spinning.

\section{Introduction}

Benefiting from its versatility, small equipment footprint, and ease in combining multiple materials within one and the same fiber, electrospinning ${ }^{[1-5]}$ has become a popular technique for producing nonwoven mats of responsive and functionalized polymer fibers with very high specific surface area. ${ }^{[6,7]}$ The diversity of properties is greatly expanded by incorporating functional liquids that are not spinnable on their own to make multifunctional composite fibers, ${ }^{[8-18]}$ using coaxial electrospinning ${ }^{[19-22]}$ or in situ phase separation. ${ }^{[23-25]}$ Exhibiting manifold properties and significant tunability, these fibers are attractive to apply across many areas, for example, in sensing, ${ }^{[12,15,26-29]}$ sound damping, ${ }^{[30]}$ dynamic patterning, ${ }^{[31]}$ thermal insulation via phase-change materials, ${ }^{[32,33]}$ self-healing coatings, ${ }^{[34]}$ or drug release. ${ }^{[35-39]}$ However, success in spinning multifunctional composite fibers requires careful optimization of the polymer solvent with respect to the functional additive: otherwise, phenomena such as phase separation and gelation can disrupt the electrospinning process. ${ }^{[6,40]}$ A common strategy (also in single-phase spinning) is to use mixtures of solvents, but many papers report only a fix solvent composition, without including any systematic study of how the polymer solution properties change with solvent mixing ratio. Given that a change in solvent composition can strongly affect the polymer-solvent interactions, there is good reason to carry out such a systematic study.

In this context, poly(acrylic acid) (PAA, Figure 1) is interesting, as it is readily soluble in water as well as ethanol, ${ }^{[41,42]}$ allowing a complete study of how mixing these two solvents affects the PAA solution and its electrospinnability. Moreover, PAA is available in high molar mass suitable for electrospinning (as confirmed by several groups reporting successful PAA nano/microfiber production ${ }^{[43-45]}$ ) and it can be made insoluble after spinning via chemical crosslinking, ${ }^{[43,44]}$ of value for making fiber mats that withstand liquid immersion. The system of PAA dissolved in a water-alcohol mixture is also highly illustrative of the complexities that can arise with polymers dissolved in mixed solvents.

DOI: $10.1002 /$ mame.202100640 


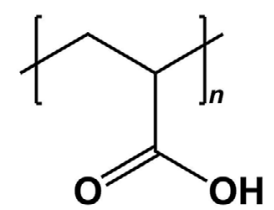

Figure 1. The structure of poly(acrylic acid) (PAA).

Studying water-ethanol solutions of polyvinylpyrrolidone (PVP), Guettari et al. ${ }^{[46]}$ found that pure ethanol is a good solvent and water is a theta solvent (at the border between good and bad), while mixing reduced the performance in both directions, leading to a maximally poor solvent at about $70 \mathrm{~mol} \%$ water. The same phenomenon was seen with water-methanol solutions of polyethylene oxide (PEO) by Shankar et al., ${ }^{[4]}$ who also demonstrated that the minimum of solvent quality coincides with a minimum of viscosity of the polymer solution. Both articles attributed the behavior to the solvent mixtures exhibiting a maximum deviation from ideal mixing behavior at a 2:1 molar ratio of water to alcohol due to competitive hydrogen bonding and formation of clusters with different solvent compositions at the molecular scale. In contrast to PVP and PEO, PAA is a polyion, that is, its pendant groups (carboxyl) are ionizable, and this can yield an even stronger sensitivity to the solvent properties. The high dielectric permittivity $\varepsilon_{\mathrm{w}} \approx 78$ of water generally leaves polyions largely ionized, ${ }^{[48]}$ giving rise to polyelectrolyte behavior. ${ }^{[49]}$ Ethanol has a much lower permittivity than water, $\varepsilon_{\mathrm{e}} \approx 25$, and we can thus expect significant counterion condensation and largely neutral PAA if it is dissolved in ethanol.

An interesting interplay between multiple phenomena should thus take place when electrospinning PAA dissolved in waterethanol mixtures of varying compositions, because of the combined effects of a solvent changing character in a nonlinear way and the change from polyelectrolyte to neutral behavior of the solute. As a primary probe of solvent quality, we measure the shear viscosity $\eta$ at low shear rate as a function of ethanol-water mixing ratio, confirming highly nonlinear behavior. To our surprise, however, PAA behaves opposite to PVP and PEO, with a maximum in $\eta$ at intermediate solvent composition. This indicates that the mixed solvent has optimum properties for dissolving PAA, a finding that we attribute to the polyelectrolyte character of PAA when water is present in the solvent. We attempt to identify a PAA concentration $c_{\mathrm{PAA}}$ for each solvent composition that yields a value of $\eta$ appropriate for electrospinning. The resulting variation in PAA concentration reveals the impact of yet another characteristic of PAA: its highly hygroscopic nature leads to significant water condensation from the air at high $c_{\mathrm{PAA}}$, changing the ethanol-water balance in the Taylor cone, with strong impact on electrospinnability.

\section{Results}

\subsection{Viscosity of PAA Solutions}

The molar mass of our PAA is the same as that used by Li and Hsieh, who measured $\eta$ as a function of $c_{\mathrm{PAA}}$ in pure water. ${ }^{[43]}$ Replotting their data on a log-log scale (Figure S2, Supporting
Information), a change of slope is easily recognized when $c_{\text {PAA }}$ increases from $5 \%$ to $6 \% \mathrm{w} / \mathrm{w}$. Fitting a power law function to the data, we find that the $\eta\left(c_{\mathrm{PAA}}\right)$ data are well reproduced with an exponent of 2 for $c_{\text {PAA }}$ in the range $2-5 \% \mathrm{w} / \mathrm{w}$, while, in the range $6-10 \% \mathrm{w} / \mathrm{w}$, the exponent is 4.1 . These exponents are well in line with the expectations for semidilute unentangled to semidilute entangled behavior, respectively ${ }^{[50]}$; hence we take $c^{\mathrm{e}}, \mathrm{w}_{\mathrm{PAA}} \approx 5.5 \% \mathrm{w} / \mathrm{w}$ as the critical entanglement concentration for PAA in water. This leads us to conduct our experiments with $c_{\text {PAA }} \geq 6 \% \mathrm{w} / \mathrm{w}$ in order to have entangled solutions suitable for electrospinning.

We prepare solutions with $c_{\mathrm{PAA}}=6 \%, 7 \%, 8 \%$, and $9 \% \mathrm{w} / \mathrm{w}$ in water-ethanol mixtures at $0 \%, 25 \%, 50 \%, 75 \%$, and $100 \% \mathrm{w} / \mathrm{w}$ water, respectively, and measure $\eta$ in a plate-plate rheometer as a function of oscillatory shear rate $\dot{\gamma}$. The full data are shown in the Supporting Information, Figures S3-S8, Supporting Information. Based on these data, we consider $\eta\left(\dot{\gamma}=2.34 \mathrm{~s}^{-1}\right)$ a representative low-shear viscosity; lower $\dot{\gamma}$ gives poor measuring accuracy, possibly related to uncontrolled solvent evaporation during the long measuring time, with fluctuations in the apparent $\eta$. To get an overview of the behavior as function of water-ethanol molar ratio $x_{\mathrm{w}}\left(x_{\mathrm{w}}=0\right.$ corresponding to pure ethanol) as well as of $c_{\text {PAA }}$, we plot $\eta\left(\dot{\gamma}=2.34 \mathrm{~s}^{-1}\right)$ as a function of $x_{\mathrm{w}}$ in Figure 2 for $c_{\text {PAA }}=6,7$, and $9 \% \mathrm{w} / \mathrm{w}$, respectively (see Supporting Information concerning the $8 \% \mathrm{w} / \mathrm{w}$ data). As an attempt to extrapolate between the experimental data points, we fitted a single-peak Gaussian function to each data set, yielding a good match to the obtained data.

The striking conclusion from Figure 2 is that all systems show a clear maximum in $\eta$ for $x_{\mathrm{w}} \approx 0.65$. While we thus can reproduce the findings of Guettari et al. ${ }^{[46]}$ and Shankar et al. ${ }^{[47]}$ in terms of extreme behavior at a water-alcohol mole ratio of 2:1, corresponding to maximally nonideal behavior of the solvent, we see the opposite extreme of maximum viscosity, suggesting maximum coil expansion and thus a maximally good solvent at this mixing ratio. We will return to the interpretation of this difference in the Discussion.

Given the strong variation in viscosity with water-ethanol ratio, it is clear that a $c_{\mathrm{PAA}}$ value suitable for electrospinning with one solvent composition may no longer be adequate if the waterethanol ratio changes. We hypothesize that it will be possible to spin fibers with consistent quality across the full water-ethanol mixture window if we identify values of $c_{\text {PAA }}$ for each solvent composition that yield a common viscosity $\eta_{0}$, which is tuned to be appropriate for electrospinning. We identify $\eta_{0}$ empirically by conducting preliminary electrospinning experiments using a solvent with water-ethanol ratio of 50:50 (by mass) and varying $c_{\text {PAA }}$. The best results (fibers with uniform diameter and few beads) are obtained with $7 \% \mathrm{w} / \mathrm{w}$ PAA, corresponding to $\eta \approx 700-800 \mathrm{mPa} \cdot \mathrm{s}$ at $\dot{\gamma}=2.34 \mathrm{~s}^{-1}$. For the other solvent compositions, we thus interpolate each relevant $\eta(\dot{\gamma})$ data set corresponding to Figures S3-S7, Supporting Information using an exponential fit to identify $c_{\text {PAA }}$ that will yield $\eta_{0} \approx 700-800 \mathrm{mPa} \cdot \mathrm{s}$ at $\dot{\gamma}=2.34 \mathrm{~s}^{-1}$. Table 1 lists the compositions of the resulting PAA solutions prepared for electrospinning and Figure S9, Supporting Information shows the corresponding $\eta(\dot{\gamma})$ curves. The data for PAA in pure ethanol and in pure water, respectively, almost overlap at values slightly higher than $\eta_{0}$, while the data obtained with mixed solvents show some variations within the range $600-950 \mathrm{mPa}$. 


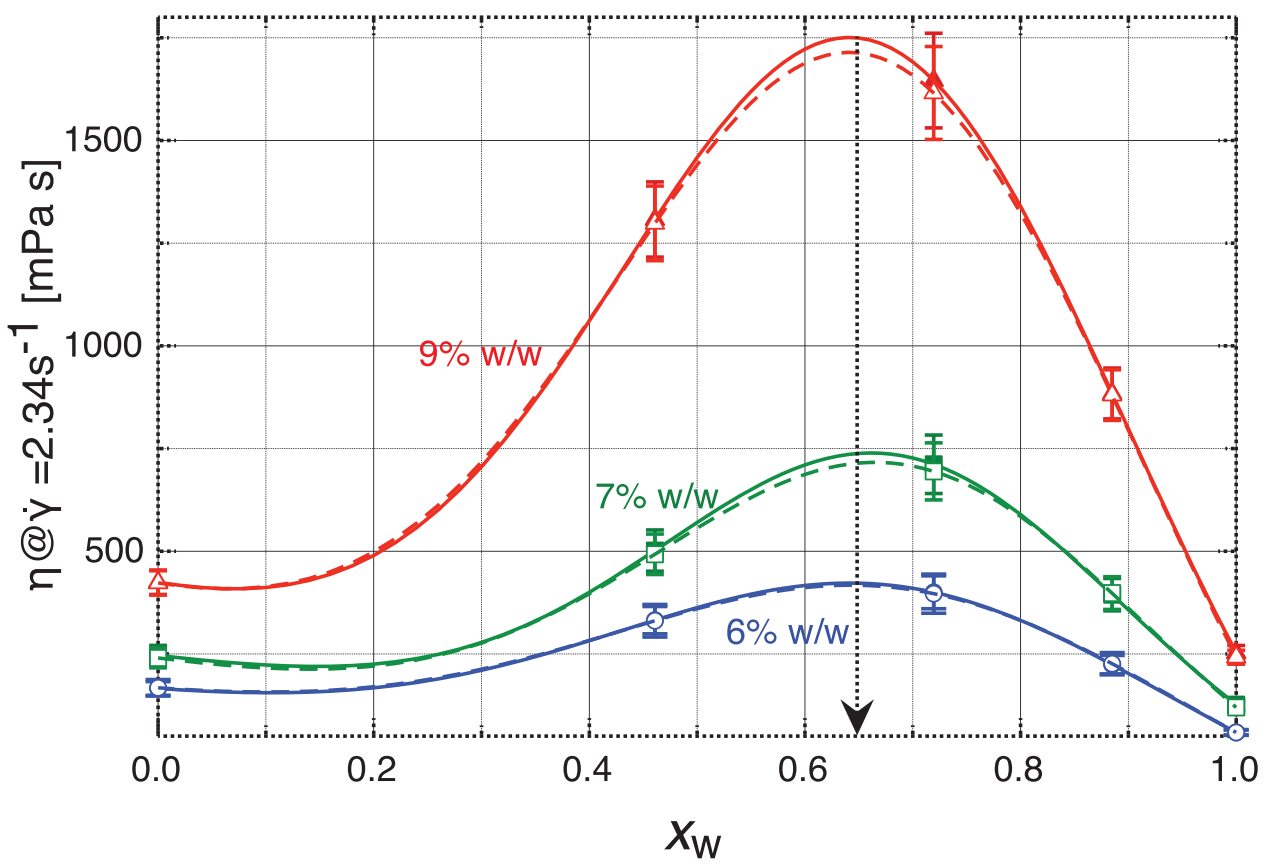

Figure 2. Low-shear rate $\left(\dot{\gamma}=2.34 \mathrm{~s}^{-1}\right)$ viscosity $\eta$ as a function of mole fraction of water in the ethanol-water solvent used for dissolving PAA at mass concentrations $6 \%, 7 \%$, and $9 \% \mathrm{w} / \mathrm{w}$, respectively. Filled symbols and continuous curves correspond to $\eta$ obtained during a scan with increasing $\dot{\gamma}$, whereas empty symbols and dashed curves correspond to measurements upon decreasing $\dot{\gamma}$. The arrow highlights the solvent composition with $n_{w} \approx 0.65$ which appears to exhibit optimum solvent properties for PAA. The error bars are a standard deviation obtained from 4 different measurements each with increasing and decreasing shear rate.

Table 1. Concentrations (by mass) $c_{\mathrm{PAA}}$ of viscosity-matched solutions with different mole fractions $x_{\mathrm{w}}$ of water (or mass fractions $c_{\mathrm{w}}$ ), both referring to the mixture without PAA, tailored for comparable electrospinning conditions regardless of solvent composition.

\begin{tabular}{lccc}
\hline Solution & $c_{\text {PAA }}[\% \mathrm{w} / \mathrm{w}]$ & $x_{\mathrm{w}}$ & $c_{\mathrm{w}}[\% \mathrm{w} / \mathrm{w}]$ \\
\hline 1 & 10 & 0 & 0 \\
2 & 7.5 & 0.46 & 25 \\
3 & 7 & 0.72 & 50 \\
4 & 8.5 & 0.88 & 75 \\
5 & 11.5 & 1 & 100 \\
\hline
\end{tabular}

\subsection{Taylor Cones and Electrospun Fibers}

After identifying the target viscosity $\eta_{0}$ and preparing PAA solutions using pure water, pure ethanol, and the three solvent mixtures, respectively, adjusting $c_{\mathrm{PAA}}$ to ensure $\eta \approx \eta_{0}$, (Table 1 ), we carried out electrospinning experiments to assess the suitability of each solution. The parameters for each spinning experiment are summarized in Table 2. During electrospinning, we paid particular attention to the Taylor cone appearance as a function of time, filming it for at least $30 \mathrm{~s}$ (Movies S1-S5, Supporting Information), and measuring the time that fibers are produced without the operator needing to clean the spinneret (we call this duration a "spinning cycle"). Snapshots from the beginning and end of a cycle, respectively, are shown in Figure 3.

Looking at the top row in Figure 3, we see that a nearideal Taylor cone develops quickly at the start of a cycle for every solu-
Table 2. The mean cross section diameter $\bar{d}$ with standard deviation, as obtained by measuring 100 individual cross sections in SEM images, of fibers produced from each density matched solution (compositions in Table 1), at relative humidity $\mathrm{RH}$, temperature $T$, spinneret-collector distance $L$, spinning voltage $V$, and flow rate $Q$.

\begin{tabular}{lcccccc}
\hline Solution & $\bar{d}[\mu \mathrm{m}]$ & $R H[\%]$ & $T\left[{ }^{\circ} \mathrm{C}\right]$ & $L[\mathrm{~cm}]$ & $V[\mathrm{kV}]$ & $Q\left[\mathrm{~mL} \mathrm{~h}^{-1}\right]$ \\
\hline 1 & $2.30 \pm 0.24$ & 29 & 25.1 & 14 & 7.5 & 0.61 \\
2 & $1.30 \pm 0.13$ & 30 & 24.8 & 14 & 7.5 & 0.65 \\
3 & $1.70 \pm 0.12$ & 30 & 24.5 & 14 & 7.5 & 0.43 \\
4 & $0.67 \pm 0.06$ & 35 & 26.3 & 14 & 7.5 & 0.23 \\
5 & $1.08 \pm 0.15$ & 35 & 25.9 & 14 & 7.5 & 0.37 \\
\hline
\end{tabular}

tion, and a stable single-jet ejection can easily be recognized in the photos. The situation is very different toward the end of a cycle, where only Solution 4 leads to a maintained stable Taylor cone and continued spinning. Although the Taylor cone in $\left(d^{\prime}\right)$ has grown in volume compared to $(d)$, an overall stable spinning situation is seen here. For the high-ethanol content solutions 1 and 2, an unnatural distortion and elongation of the Taylor cone can be recognized, suggesting that gelation is taking place on the Taylor cone surface. Indeed, this distortion is the reason why the spinneret has to be wiped clean, ending the cycle. The distortion is present also in the Taylor cone with Solution 3, although it is not as apparent, and again this is the reason for terminating the cycle by wiping the spinneret clean.

Solution 5, where pure water is used as solvent, is different. Additionally, in this experiment, the cycle is relatively short $(18 \mathrm{~s})$. 

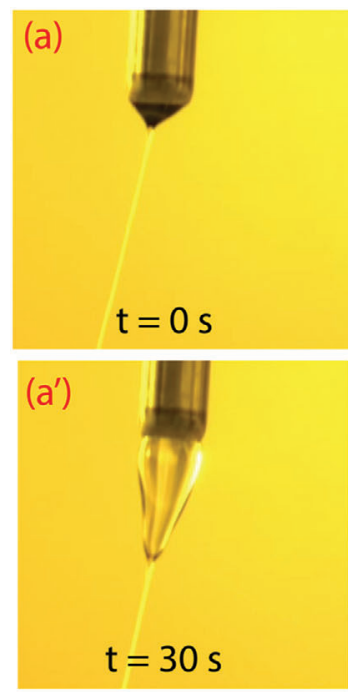

Solution 1

$\left(x_{w}=0\right)$
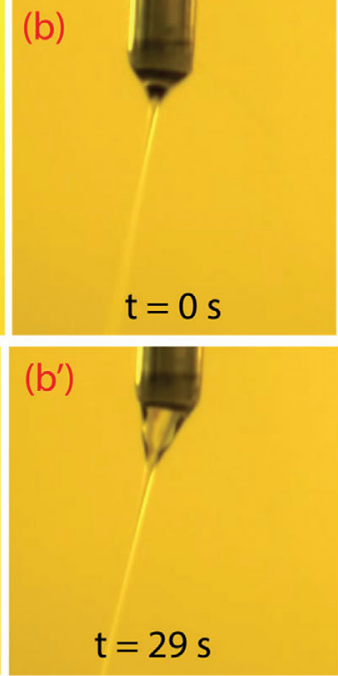

Solution 2

$\left(x_{w}=0.46\right)$
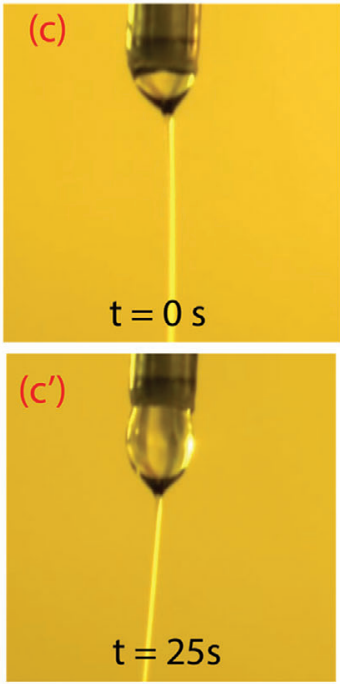

Solution 3

$\left(x_{w}=0.72\right)$
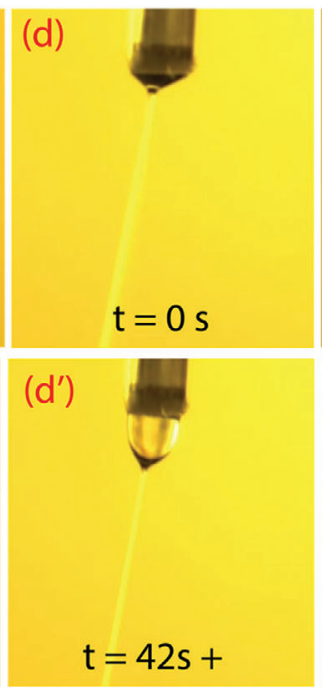

Solution 4

$\left(x_{w}=0.88\right)$
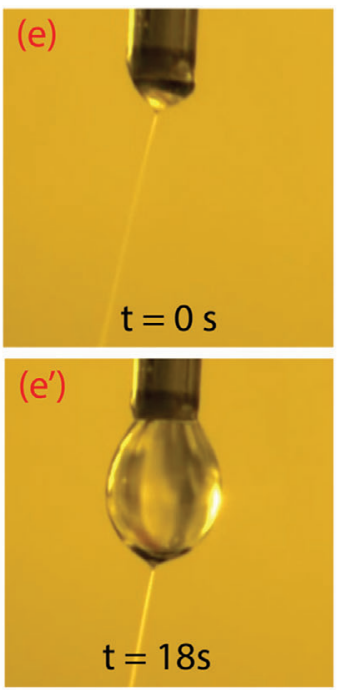

Solution 5

$\left(x_{w}=1\right)$

Figure 3. Taylor cone pictures of different PAA solutions (compositions in Table 1), imaged at the beginning (top row) and end (bottom row) of a spinning cycle. The spinneret was wiped clean at the end of each cycle, just after the timestamp label in the lower row, in order to restart the cycle, except for column $d / d^{\prime}$, where spinning could continue well beyond the $42 \mathrm{~s}$ during which the Taylor cone was filmed (hence the $t=42 \mathrm{~s}+$ label in $d^{\prime}$ )

The reason why the spinneret has to be wiped at this point is not gelation, but rather a rapid growth of the Taylor cone to a volume where it is about to detach as a droplet. If it is not wiped off, it will land on the fiber mat and ruin the previously spun fibers. We will later return to the origin of gelation of the ethanol-rich Taylor cones and of the dramatic volume expansion of the purewater-based Taylor cone (despite having the second lowest flow rate in the entire study, see Table 2 ) in the Discussion.

Our hypothesis that solutions optimized for $\eta=\eta_{0}$ will produce good fibers regardless of solvent composition is supported by the fact that all five solutions give smooth fibers that are free of beads, as shown in the scanning electron microscope (SEM) characterization images in Figure 4. However, there is a nonnegligible variation of the average diameter $\bar{d}$ of the fiber cross section, spanning the range $0.6-2.3 \mu \mathrm{m}$. As can be seen in the histograms on the right in Figure 4 and Table 2, this variation shows a systematic correlation neither with the solvent composition $x_{\mathrm{w}}$ nor with the PAA concentration $c_{\mathrm{PAA}}$. The thickest fibers are obtained with Solution 1, with a medium PAA concentration of $c_{\text {PAA }}=10 \% \mathrm{w} / \mathrm{w}$ at one end of the solvent composition scale, at $x_{\mathrm{w}}=0$, but the thinnest fibers are not obtained at the opposite end, but at $x_{\mathrm{w}}=0.88$. The fibers at $x_{\mathrm{w}}=1$, corresponding to the maximum PAA content $c_{\mathrm{PAA}}=11.5 \% \mathrm{w} / \mathrm{w}$, are among the thinner in the study, contrasting significantly to those obtained with $x_{\mathrm{w}}=0$ although these two solutions showed almost identical $\eta$ in Figure S9, Supporting Information. The flow rate $Q$ has significant impact, the thinnest fibers being spun with the lowest flow rate (Table 2), but there are inconsistencies also here. For instance, the greatest flow rate $\left(Q=0.65 \mathrm{~mL} \mathrm{~h}^{-1}\right)$ was used for Solution 2, but this produced significantly less thick fibers than Solution 1 pumped at $Q=0.61 \mathrm{~mL} \mathrm{~h}^{-1}$. We will attempt to resolve these surprising and apparent inconsistencies in the following section.

\section{Discussion}

We first address the maximum in PAA solution viscosity at intermediate water-ethanol mixing ratios, and the maximally expanded coil size that can be assumed from this behavior. The mixing of ethanol and water is a classic example of non-ideal mixing behavior, ${ }^{[51-53]}$ with a maximally negative excess volume at a water mole fraction that is often located at $x_{\mathrm{w}} \approx 0.6,{ }^{[53,54]}$ although Belda et al. report a higher value of $x_{\mathrm{w}}=0.75$. $^{[55]}$ This behavior is attributed to the clathrate caging of ethanol molecules by surrounding water molecules in order to maximize the number of hydrogen bonds of the latter, maximizing the enthalpy of mixing at the cost of reduced entropy, as the caging reduces the configuration space for hydrogen bonding of the water molecules. ${ }^{[56]}$ The maximum deviation from ideal mixing behavior can also be seen in a maximum of viscosity of ethanol-water mixtures at $x_{\mathrm{w}} \approx 0.7 \cdot{ }^{[57]}$ However, this viscosity maximum is three orders of magnitude lower than that of the PAA solutions studied here; the impact of the viscosity of the solvent itself is thus negligible in the context of our study.

We note that the most common value $x_{\mathrm{w}} \approx 0.6^{[54]}$ for maximum non-ideal water-ethanol mixing coincides rather well with $x_{\mathrm{w}} \approx 0.65$ for maximum PAA solution viscosity seen in Figure 2, as well as with the minimum of solvent quality for PVP in ethanol-water mixtures studied by Guettari et al. ${ }^{[46]}$ Based on dielectric spectroscopy data, Sato et al. concluded that a first critical mole fraction is $x_{\mathrm{w}}=0.82$, at which the excess activation free energy $€ G^{\mathrm{E}}$ is at a maximum, corresponding to maximum ethanol-water interactions. ${ }^{[52]}$ They argue that, for lower $x_{\mathrm{w}}$, ethanol molecules form clusters, thus with microphase segregation taking place between water and ethanol. They found a second critical mole fraction of $x_{\mathrm{w}}=0.58$, near the region where most studies find the maximum negative excess volume, ${ }^{[54]}$ which they 

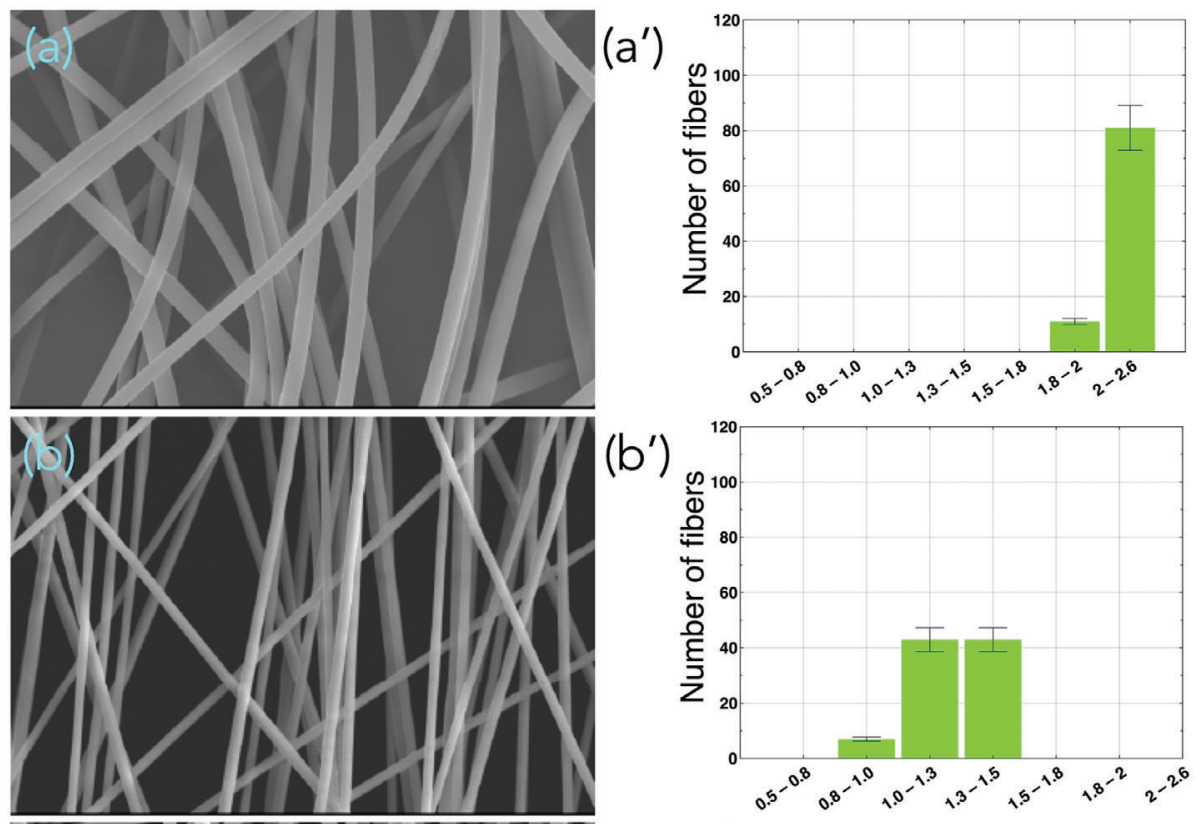

$\left(b^{\prime}\right)$
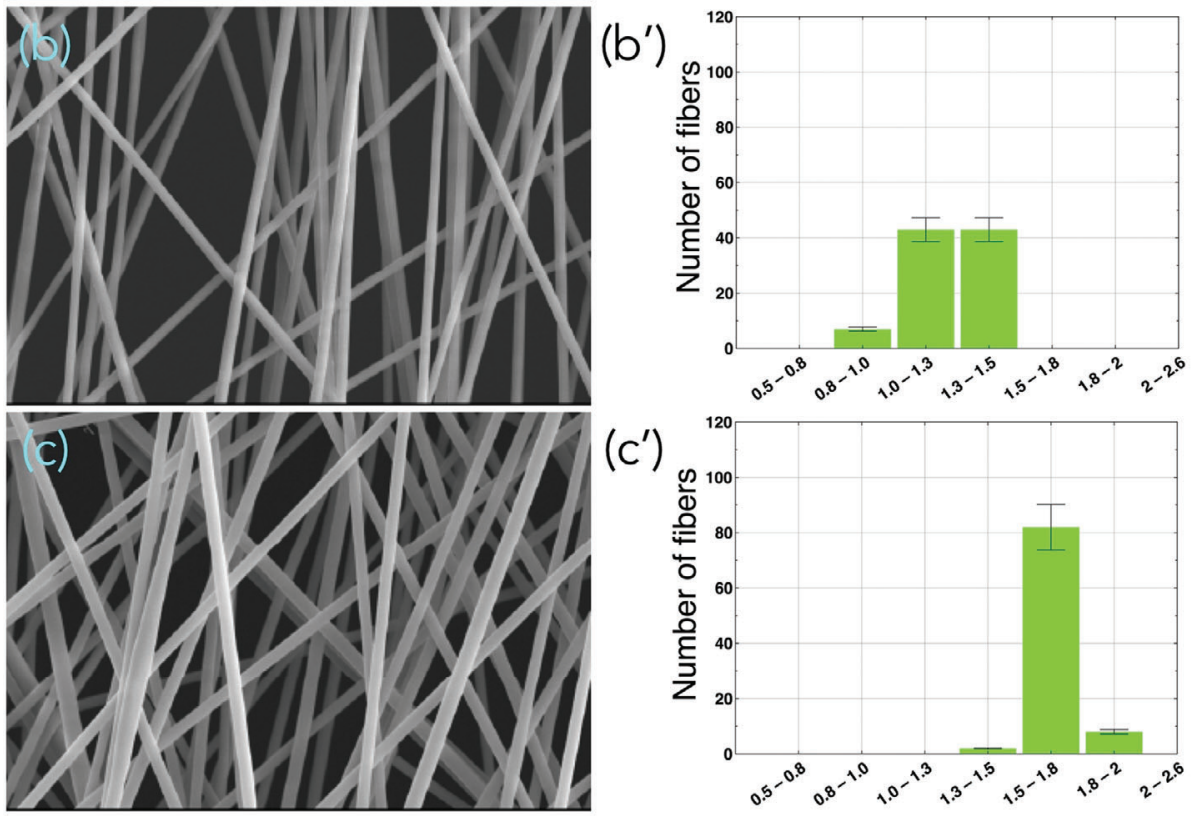

$\left(c^{\prime}\right)$
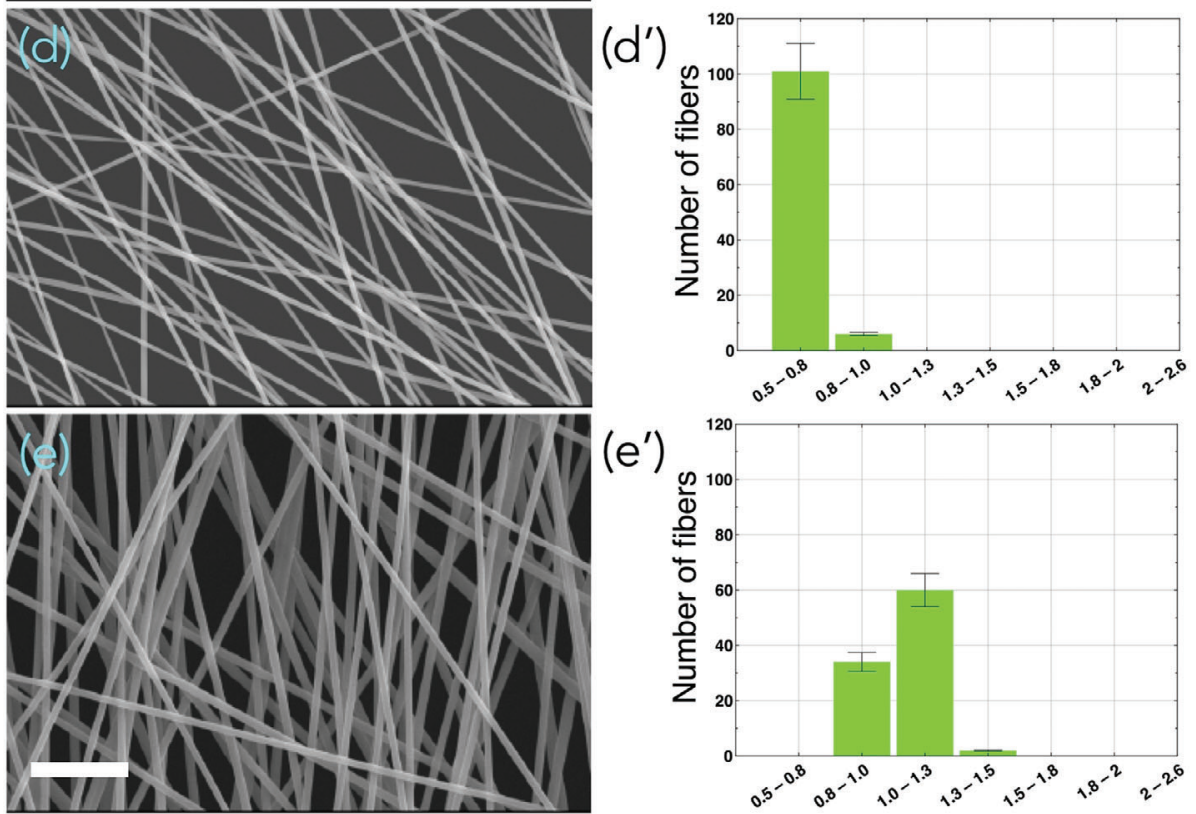

$\left(e^{\prime}\right)$
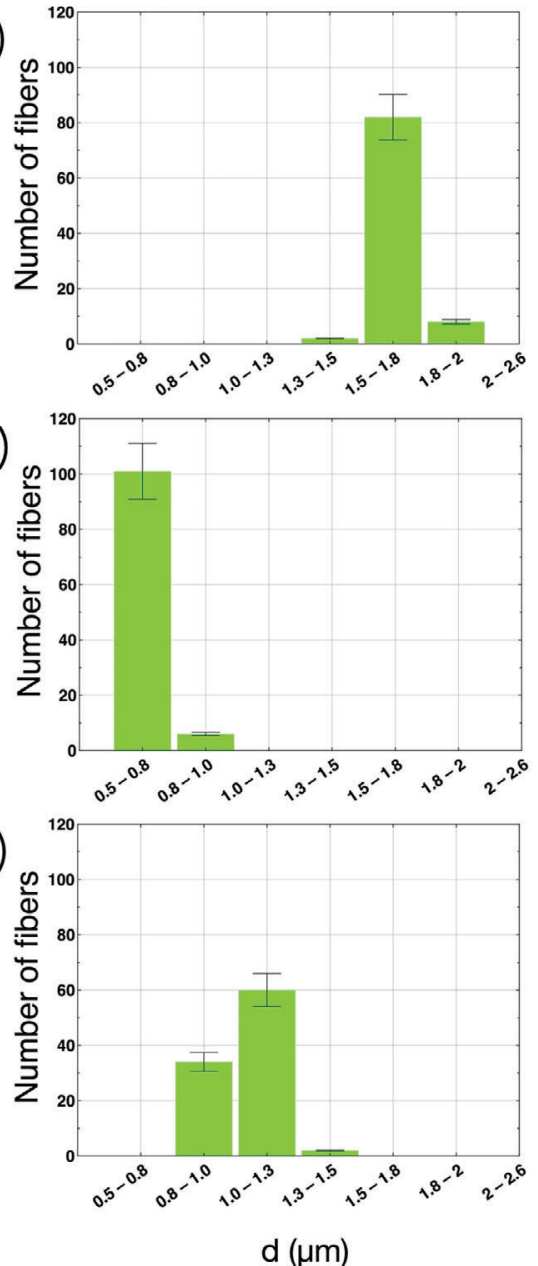

Figure 4. Left column: representative SEM images of the fibers produced from each of the five viscosity-matched solutions (compositions in Table 1). Scale bar $5 \mu \mathrm{m}$. Right column: histograms showing the distribution of cross section diameters $d$ of fibers spun from each solution. The corresponding Taylor cone movies are provided in Supporting Information, and key snapshots are shown in Figure 3. The error bars on the histograms represent standard deviation. 
attribute to this microphase segregation reaching its maximum, linking it to Ben-Naim's identification of this mole fraction as having maximum affinity of ethanol molecules but minimum affinity between ethanol and water. ${ }^{[51]}$ We can thus conclude that the mole fraction where we find a maximum PAA solution viscosity corresponds to the conditions where the ethanol-water mixture is the furthest from ideal, with a microphase segregation of water and ethanol which is at its maximum.

The key remaining question is why this extreme condition yields maximum viscosity of PAA solutions, suggesting maximally expanded coils and an optimum solution behavior for PAA, whereas the same solvent mixture shows an extreme in poor solvent behavior for PVP. ${ }^{[46]}$ We assume that this is related to the ionizable acid groups of PAA and the fact that the counter ions of the carboxyl pendants of PAA are protons $\left(\mathrm{H}^{+}\right)$, rendering the degree of ionization highly sensitive to $\mathrm{pH}$. The acidic nature of PAA thus itself influences its degree of deprotonation. For aqueous PAA solutions set to $\mathrm{pH}<5$ by a low molar mass acid, Swift et al. found the deprotonation of the carboxyl groups to be negligible. ${ }^{[58]}$ In our case, $\mathrm{pH}<5$ for $c_{\mathrm{PAA}} \geq 6 \% \mathrm{w} / \mathrm{w}$ for all $x_{\mathrm{w}}$ (see Figure S10, Supporting Information), which means that the average degree of ionization of PAA must be low, explaining why ethanol is overall the better solvent. Indeed, we find a slight turbidity in the solutions with high water content, whereas those with high ethanol content are clear (Figure S11, Supporting Information). However, the low $\mathrm{pH}$ comes from protonation of PAA in the first place; hence, ionization up to a saturation fraction does occur along the chains. This gives them polyelectrolyte character with electrostatic self repulsion along the polymer chain, increasing the persistence length and therefore expanding the coil in solution. As the ethanol fraction of the solvent increases, $\mathrm{pH}$ also increases, reflecting the expected reduced degree of ionization as the solvent permittivity decreases. This means a decreasing electrolyte character, with a consequent reduced impact of electrostatic coil expansion. The variation of PAA ionization should be reflected by a corresponding variation of solution conductivity, which would suggest that the fiber thickness increases with ethanol content, as high conductivity is known to decrease the fiber diameter. ${ }^{[1-5]}$ However, the results in Figure 4 show a dependence on solvent composition that is not fully systematic, suggesting that the conductivity impact is overlaid by other effects, to be discussed below.

We conjecture that the maximum viscosity at constant $c_{\mathrm{PAA}}$ at $n_{\mathrm{w}} \approx 0.65$ is a result of maximally expanded coils because of all the different aspects influencing the PAA chain interactions contributing synergistically at this apparently optimum solvent mixture composition. The benefit of water's ability to deprotonate the PAA is combined with ethanol's ability to solubilize the protonated residues (in majority), and the partial charging of the polymer expands the coil via the electrostatically increased persistence length. These effects are then amplified by the fact that the entropic penalty of microphase segregation of ethanol and water is maximized at $w_{\mathrm{x}} \approx 0.6 .^{[52]}$ The presence of partially ionized PAA could reduce the free energy of the solvent by having the water molecules interacting mainly with deprotonated acid groups while the ethanol interacts mainly with the protonated ones and the polymer backbone, as has indeed been suggested by molecular dynamics simulations. ${ }^{[59]}$ In other words, the polymer-solvent interactions are particularly strong at $w_{\mathrm{x}} \approx 0.6$, as each fraction of the polymer, protonated and deprotonated, respectively, interacts preferentially with one of the two solvent species, reducing the entropy penalty of direct ethanolwater interaction at this maximally nonideal mixing ratio. Hammouda et al. also found that a mixture of ethanol and water promotes PAA dissolution ${ }^{[60]}$ and they also attributed the effect to a molecular scale solvent segregation. However, their conjecture that water dissolves mainly the carboxylate groups-regardless of degree of deprotonation-whereas the ethanol dissolves mainly the backbone would suggest that water-alcohol mixtures would be better solvents also for PVP and PEO, in contradiction with the findings of Guettari et al. ${ }^{[46]}$ and Shankar et al. ${ }^{[47]}$ Future more detailed investigations, in particular involving computer simulations, are needed to gain a full understanding of the phenomena.

Next, we attempt to explain the apparently unsystematic variations in fiber cross section diameter $\bar{d}$ between the five solutions used for electrospinning seen in Figure 4 and Table 2. We believe that this is due to the fact that the impact on the electrospinning results of $\eta$-which is similar for all five solutions as they were prepared-is dwarfed by the changes to the solvent composition taking place over time in the Taylor cone. This thus gives rise to the significant variations in cycle length and Taylor cone shape seen in Figure 3, but it also impacts the characteristics of the fibers that finally end up on the collector. We recently found that the cooling due to ethanol evaporation from the Taylor cone of an ethanolic PVP solution electrospun in a humid atmosphere leads to significant water condensation, which-counterintuitivelyleads to gelation of the Taylor cone surface and disruption of the electrospinning process. ${ }^{[40]}$ This surprising finding can be understood from the fact that water condensation actually accelerates ethanol or methanol evaporation due to the latent heat released when water condenses. ${ }^{[61]}$

We believe the same phenomenon is at play here, despite a significantly lower atmospheric humidity. Most likely, the wellknown hygroscopic nature of PAA (the most common application of PAA is as a superabsorbant material due to its ability to absorb water and swell to many times its own volume) contributes to an extreme sensitivity to humidity, the PAA effectively promoting water condensation and thus triggering the enhanced ethanol evaporation. This explains why the outer surface of the Taylor cone of PAA in pure ethanol gels so quickly, terminating the spinning cycle after about $30 \mathrm{~s}$ in Figure 3, and it also explains why the Taylor cone always grows in volume over time within a spinning cycle, despite the flow rate being slightly lower than typical for electrospinning. In fact, $Q$ was as low as possible in all experiments, any further reduction effectively stopping the initial Taylor cone development. If water is present in the spinning solution from the start, the impact on ethanol evaporation is reduced in magnitude, but the gelation of the Taylor cone is nevertheless strong enough to also terminate the cycle in spinning experiments with Solutions 2 and 3.

With Solution 5, there is no ethanol that can evaporate, as the solvent is pure water. In this case, consequently, the cycle is not terminated by surface gelation of the Taylor cone, but by an extreme growth of the droplet protruding from the spinneret to the point that it can hardly be described as a Taylor cone any more and where an operator must remove it in order to avoid dripping onto the fiber mat. It must be emphasized that this is not due to overfeeding because of too high flow rate; $Q$ is the second lowest in 
the study for Solution 5 . Rather, we believe it is the fact that $c_{\mathrm{PAA}}$ is the highest of all solutions, in order to reach the targeted $\eta_{0}$ in this nonoptimum solvent. The large PAA loading leads to maximum impact of the hygroscopic nature of PAA, drawing in additional water from the air as spinning proceeds. While it may seem surprising that PAA could act to draw in additional water from the air when it already is in solution, one should be aware that significant phase separation takes place during electrospinning in the Taylor cone, near the apex from which the jet is ejected. ${ }^{[62]}$ This means that the PAA concentration can be high locally, allowing it to absorb additional water at these locations.

We believe the low value of $\bar{d}$ seen when spinning solution 4 is the one truly representative of the viscosity-matched solutions. This solution has a quite low $c_{\mathrm{PAA}}$, reducing the impact of its hygroscopic nature, and a relatively low ethanol content, eliminating the risk of Taylor cone gelation due to water condensation. The other fibers have $\bar{d}$ that are more determined by the dynamic variations of the PAA solution at the Taylor cone surface during spinning, due to water condensing from the air.

\section{Conclusion}

While PAA dissolves in ethanol as well as water, or any mixture of the two, its behavior changes in a highly nonlinear and nontrivial way as the solvent composition is varied. Surprisingly, the viscosity $\eta$ at constant PAA concentration $c_{\text {PAA }}$ is maximum at a waterto-ethanol mole fraction of $x_{\mathrm{w}} \approx 0.65$, corresponding to maximum deviation from ideal mixing behavior of water and ethanol. We believe it provides a synergistic combination of partial deprotationation of carboxylate groups by water while the ethanol dissolves the remaining uncharged polymer fraction. Having established how $\eta$ varies with $x_{\mathrm{w}}$ we find that consistent electrospinning of PAA solutions of any water-ethanol solvent ratio can be initiated with $\eta \approx 0.7 \mathrm{~Pa} \mathrm{~s}$, but the fiber cross section diameter varies in apparently inconsistent ways, and the spinneret must be regularly wiped clean, due to an unavoidable rapid growth of the Taylor cone and/or gelation in case of ethanol-rich solutions. We attribute this behavior to the extreme hygroscopic nature of PAA, leading to significant water condensation into the Taylor cone despite a spinning atmosphere with moderate relative humidity. This demonstrates the vital importance of monitoring the Taylor cone quality over time during electrospinning and paying attention to any unexpected processes taking place there, which may impact the electrospinning process.

\section{Experimental Section}

Polymer Solutions: Poly (acrylic acid) (PAA; $M_{w}=450 \mathrm{~kg} \mathrm{~mol}^{-1}$ ) was purchased from Sigma-Aldrich and dissolved in anhydrous ethanol $(99 \%$, from VWR) for solutions in pure ethanol, ultrapure deionized water (conductivity $0.055 \mu \mathrm{S} \mathrm{cm}^{-1}$ Sartorius Arium) for solutions in pure water, or mixtures of the two solvents. Multiple PAA solutions were prepared, with various selected concentrations $C_{\text {PAA }}$ (by mass), in water-ethanol mixtures of $0,25,50,75$, and $100 \% \mathrm{w} / \mathrm{w}$ ratio. All materials were used as received without further purification.

Viscosity Measurements: The oscillatory shear viscosity $\eta$ was measured in plate-plate geometry (50 mm diameter, $1.0 \mathrm{~mm}$ gap) using an Anton Paar rheometer (MRC-102), controlled by Rheocompass software. The shear rate $\dot{\gamma}$ was swept from 0.1 to $10 \mathrm{~s}^{-1}$ and then back to $0.1 \mathrm{~s}^{-1}$. All the measurements were performed at room temperature $\left(25^{\circ} \mathrm{C}\right)$, using a solvent trap to prevent ethanol from evaporating during the measurements.

Electrospinning: The electrospinning setup (vertical geometry, schematic in Figure S1, Supporting Information) was housed inside a closed acrylic box in order to ensure a well-defined spinning atmosphere. An 18-gauge blunt-tipped disposable stainless steel needle (outer diameter $1.20 \mathrm{~mm}$ ), purchased from VWR, was used as the spinneret, mounted in the top of the acrylic box. To apply the electric field to drive spinning, the spinneret was connected to a high voltage power supply (Gamma High Voltage, model ES30R-5W/DAM/RS232) while the collector was grounded. The PAA solution to be spun was pumped to the spinneret using a microfluidic pressure controller (Fluigent, model MFCS-EZ, maximum pressure 1034 mbar, uncertainty \pm 0.3 mbar). The Taylor cone was imaged using a digital camera (Pixelink D755) equipped with a macro lens (Tokina AT-X Pro).

For all experiments, a spinneret-collector distance of $14 \mathrm{~cm}$ and a spinning voltage of $7.5 \mathrm{kV}$ were chosen. The temperature $T$ and relative humidity $\mathrm{RH}$ were monitored during all experiments, and the flow rate $Q$ was adjusted for maximally stable spinning for each solution. Fibers were collected freely hanging on an untreated copper wire frame.

Electron Microscopy Characterization: The fibers were characterized using a JEOL JSM-6010LA SEM (Akishima, Japan), operated at $20 \mathrm{kV}$ with a working distance of $11 \mathrm{~mm}$. Prior to imaging, the fibers were coated with gold ( $\approx 5 \mathrm{~nm}$ thickness) using a sputter coater (Quorum Q150R ES) for $100 \mathrm{~s}$. The fiber cross section diameter was established by randomly selecting at least 100 fibers from the SEM images and measuring the apparent cross section using the software Image ( $\mathrm{NIH}$, USA).

\section{Supporting Information}

Supporting Information is available from the Wiley Online Library or from the author.

\section{Acknowledgements}

Funding for this research was provided by a European Research Council Consolidator Grant (INTERACT, grant number 648763) and by an Aide à la formation-recherche grant (LIMEFLOW, grant number 9784104) from the Luxembourg National Research Fund. The authors thank Nicolas Tournier, Dr. Ulrich M. Siegel, Dr. Catherine G. Reyes, and Dr. Hakam Agha for assistance in constructing the experimental set-up; Dr. Alex Gansen, Claudius M. Lehr, Dr. Jörg Baller, and Prof. Jeanne H. Norton for assistance with the rheological characterization; Zornitza Tosheva for helping with SEM imaging and $\mathrm{pH}$ measurements; and Dr. Manos Anyfantakis and Dr. V.S.R. Jampani for fruitful discussions.

\section{Conflict of Interest}

The authors declare no conflict of interest.

\section{Data Availability Statement}

The data that support the findings of this study are available from the corresponding author upon reasonable request.

\section{Keywords}

electrospinning, ethanol, poly(acrylic acid), polymers, solvents, viscosity, water

Received: August 27, 2021

Revised: October 29, 2021

Published online: 
[1] S. Ramakrishna, K. Fujihara, W.-E. Teo, T.-C. Lim, Z. Ma, An Introduction to Electrospinning and Nanofibers Electrospinning Process, Vol. 3, World Scientific, Singapore 2005.

[2] G. Rutledge, S. Fridrikh, Adv. Drug Delivery Rev. 2007, 59, 1384.

[3] D. Reneker, A. Yarin, Polymer 2008, 49, 2387.

[4] J. H. Wendorff, S. Agarwal, A. Greiner, Electrospinning: Materials, Processing, and Applications, Wiley-VCH, Weinheim 2012.

[5] J. Xue, T. Wu, Y. Dai, Y. Xia, Chem. Rev. 2019.

[6] M. Urbanski, C. G. Reyes, J. Noh, A. Sharma, Y. Geng, V. S. R. Jampani, J. P. F. Lagerwall, J. Phys.: Condens. Matter 2017, 29, 133003.

[7] J. H. Park, G. C. Rutledge, Macromolecules 2017, 50, 5627.

[8] M. Jasiurkowska-Delaporte, E. Juszyńska-Gałązka, W. Sas, P. M. Zieliński, A. Baranowska-Korczyc, J. Mol. Liq. 2021, 331, 115817.

[9] A. E. Mamuk, Ç. Koçak, Ç. E. Demirci Dönmez, Colloid Polym. Sci. 2021.

[10] K. T. Dicker, D. Ratchford, R. Casalini, M. D. Thum, J. H. Wynne, J. G. Lundin, Langmuir 2020, 36, 7916.

[11] G. Scalia, E. Enz, O. Calò, D. K. Kim, M. Hwang, J. H. Lee, J. P. F. Lagerwall, Macromol. Mater. Eng. 2013, 298, 583.

[12] D. K. Kim, M. Hwang, J. P. F. Lagerwall, J. Polym. Sci., Part B: Polym. Phys. 2013, 51, 855 .

[13] E. Enz, V. La Ferrara, G. Scalia, ACS Nano 2013, 7, 6627.

[14] A. L. Medina-Castillo, J. F. Fernández-Sánchez, A. FernándezGutiérez, Adv. Funct. Mater. 2011, 21, 3488.

[15] E. Enz, J. P. F. Lagerwall, J. Mater. Chem. 2010, 20, 6866.

[16] E. Enz, U. Baumeister, J. Lagerwall, Beilstein J. Org. Chem. 2009, 5, 58.

[17] J. P. F. Lagerwall, J. T. McCann, E. Formo, G. Scalia, Y. Xia, Chem. Commun. 2008, 42, 5420.

[18] J. E. Díaz, A. Barrero, M. Márquez, I. G. Loscertales, Adv. Funct. Mater. 2006, 16, 2110 .

[19] P. Rathore, J. Schiffman, ACS Appl. Mater. Interfaces 2021, 13, 48.

[20] D. Han, A. Steckl, Chempluschem 2019, 84, 1453.

[21] A. Yarin, Polym. Adv. Technol. 2011, 22, 310.

[22] L. Bellan, H. Craighead, Polym. Adv. Technol. 2011, 22, 304.

[23] J. Wang, A. Jákli, J. L. West, J. Mol. Liq. 2018, 267, 490.

[24] J. Wang, A. Jákli, J. West, ChemPhysChem 2016, 17, 3080.

[25] E. A. Buyuktanir, M. W. Frey, J. L. West, Polymer 2010, 51, 4823.

[26] S. Ji, Y. Li, M. Yang, Sens. Actuators, B 2008, 133, 644.

[27] Y. Kye, C. Kim, J. P. F. Lagerwall, J. Mater. Chem. C 2015, 3, 8979.

[28] C. G. Reyes, A. Sharma, J. P. F. Lagerwall, Liq. Cryst. 2016, 43, 1986.

[29] L. Pschyklenk, T. Wagner, A. Lorenz, P. Kaul, ACS Appl. Polym. Mater. 2020, 2, 1925

[30] M. J. Bertocchi, P. Vang, R. B. Balow, J. H. Wynne, J. G. Lundin, ACS Appl. Polym. Mater. 2019, 1, 2068.

[31] M. D. Thum, D. C. Ratchford, R. Casalini, J. H. Wynne, J. G. Lundin, ACS Appl. Nano Mater. 2021, 4, 297.

[32] N. Wang, H. Chen, L. Lin, Y. Zhao, X. Cao, Y. Song, L. Jiang, Macromol. Rapid Commun. 2010, 31, 1622.
[33] J. McCann, M. Marquez, Y. Xia, Nano. Lett. 2006, 6, 2868.

[34] J. Park, P. Braun, Adv. Mater. 2009, 22, 496.

[35] B. Pant, M. Park, S.-J. Park, Pharmaceutics 2019, 11, 305.

[36] K. Khoshnevisan, H. Maleki, H. Samadian, S. Shahsavari, M. Sarrafzadeh, B. Larijani, F. Dorkoosh, V. Haghpanah, M. Khorramizadeh, Carbohydr. Polym. 2018, 198, 131.

[37] S. Chou, D. Carson, K. Woodrow, J. Controlled Release 2015, 220, 584.

[38] W. Ji, Y. Sun, F. Yang, J. van den Beucken, M. Fan, Z. Chen, J. Jansen, Pharm. Res. 2011, 28, 1259.

[39] A. Saraf, L. Baggett, R. Raphael, F. Kasper, A. Mikos, J. Controlled Release 2010, 143, 95.

[40] C. G. Reyes, J. P. Lagerwall, ACS Appl. Mater. Interfaces 2020, 12, 26566.

[41] K. Terao, in Encyclopedia of Polymeric Nanomaterials (Eds: S. Kobayashi, K. Müllen), Springer, Berlin, Heidelberg 2021, pp. 1-6.

[42] J. O. Iroh, in Polymer Data Handbook (Ed: J. Mark), Oxford University Press, New York 1999, pp. 558-560.

[43] L. Li, Y. Hsieh, Nanotechnology 2005, 16, 2852

[44] Y. B. Truong, J. Choi, J. Mardel, Y. Gao, S. Maisch, M. Musameh, I. L. Kyratzis, Macromol. Mater. Eng. 2017, 302, 1700024.

[45] I. Ismail, N. Bakar, T. Ling, N. Ideris, Z. Zain, N. Radacsi, Mater. Today: Proc. 2019, 17, 574.

[46] M. Guettari, A. Belaidi, S. Abel, T. Tajouri, J. Solution Chem. 2017, 46, 1404.

[47] R. Shankar, R. R. Klossner, J. T. Weaver, T. Koga, J. H. van Zanten, W. E. Krause, C. M. Colina, F. Tanaka, R. J. Spontak, Soft Matter 2009, 5, 304.

[48] G. Smolyakov, J.-M. Catala, N. Kutsevol, M. Rawiso, in Modern Problems of Molecular Physics (Eds: L. A. Bulavin, A. V. Chalyi), Springer, Cham 2018, pp. 133-147.

[49] X. Meng, Y. Du, Y. Liu, E. B. Coughlin, S. L. Perry, J. D. Schiffman, Macromolecules 2021, 54, 5033.

[50] S. K. Tiwari, S. S. Venkatraman, Mater. Sci. Eng.: C 2012, 32, 1037.

[51] A. Ben-Naim, J. Chem. Phys. 1977, 67, 4884.

[52] T. Sato, A. Chiba, R. Nozaki, J. Chem. Phys. 1999, 110, 2508.

[53] J. Ott, J. Sipowska, M. Gruszkiewicz, A. Woolley, J. Chem. Thermodyn. 1993, 25, 307.

[54] I. Abdulagatov, L. A. Akhmedova-Azizova, N. Azizov, Fluid Phase Equilib. 2014, 376, 1 .

[55] R. Belda, J. V. Herráez, O. Diez, Phys. Chem. Liq. 2004, 42, 467.

[56] D. Chandler, Nature 2005, 437, 640.

[57] S. Song, C. Peng, J. Dispersion Sci. Technol. 2008, 29, 1367.

[58] T. Swift, L. Swanson, M. Geoghegan, S. Rimmer, Soft Matter 2016, 12,2542

[59] S. Srikant, S. S. Muralidharan, U. Natarajan, Mol. Simul. 2013, 39, 145.

[60] B. Hammouda, F. Horkay, M. L. Becker, Macromolecules 2005, 38, 2019.

[61] C. Law, T. Xiong, C. Wang, Int. J. Heat Mass Transfer 1987, 30, 1435.

[62] C. Wang, T. Hashimoto, Macromolecules 2020, 53, 9584. 


\section{Supplementary Information for "Electrospinning ethanol-water solutions of poly(acrylic acid): non-linear viscosity variations and sensitivity to air humidity"}

Shameek Vats, Lawrence W. Honaker, Margaret W. Frey, Francesco Basoli, and Jan P.F. Lagerwall

\section{A Electrospinning set-up and conditions}

Figure S1 shows a schematic of the electrospinning setup discussed in the Experimental section and used for these experiments.

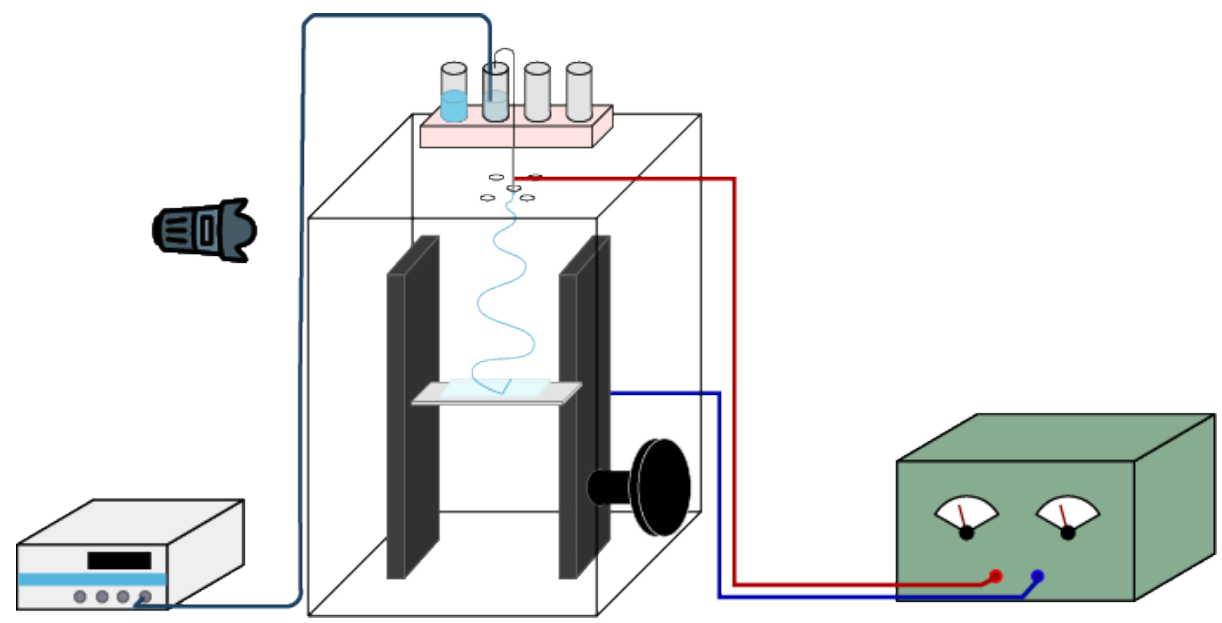

Figure S1: The schematics of the setup used for electrospinning microfibers

The parameters under which spinning took place are shown in Table S1.

Table S1: Electrospinning parameters and conditions

\begin{tabular}{|c|c|c|c|c|c|}
\hline $\begin{array}{c}\text { PAA } \\
\text { Concentration }\end{array}$ & $\begin{array}{c}\text { Relative } \\
\text { Humidity }(\%)\end{array}$ & $\begin{array}{c}\text { Temperature } \\
\left({ }^{\circ} \mathrm{C}\right)\end{array}$ & $\begin{array}{c}\text { Distance } \\
(\mathrm{cm})\end{array}$ & $\begin{array}{c}\text { Voltage } \\
(\mathrm{kV})\end{array}$ & $\begin{array}{c}\text { Flow rate } \\
(\mathrm{ml} / \mathrm{h})\end{array}$ \\
\hline $10 \% \mathrm{w} / \mathrm{w}$ & 29 & 25.1 & 14 & 7.5 & 0.61 \\
\hline $7.5 \% \mathrm{w} / \mathrm{w}$ & 30 & 24.8 & 14 & 7.5 & 0.65 \\
\hline $7 \% \mathrm{w} / \mathrm{w}$ & 30 & 24.5 & 14 & 7.5 & 0.43 \\
\hline $8.5 \% \mathrm{w} / \mathrm{w}$ & 35 & 26.3 & 14 & 7.5 & 0.23 \\
\hline $11.5 \% \mathrm{w} / \mathrm{w}$ & 35 & 25.9 & 14 & 7.5 & 0.37 \\
\hline
\end{tabular}

The flow rates indicated in Table S1 were calculated from the Hagen-Poiseuille equation (equation 1) for the pressure drop between two ends of a pipe or tube:

$$
\Delta p=\frac{8 \eta L Q}{\pi R^{4}}
$$

where $\Delta p$ is the pressure difference between the ends of the tube, $Q$ the volumetric flow rate, $\eta$ the viscosity, $R$ the cross-sectional radius of the tube, and $L$ its length. Simple rearrangement allows us to express $Q$ in terms of the input parameters (most notably, $\Delta p, L$, and $R$ ):

$$
Q=\frac{\Delta p \pi R^{4}}{8 \eta L}
$$

The parameters used in equation 2 to calculate the flow rates are indicated in Table S2.

\section{B PAA solutions and their viscosities}

To establish the critical entanglement concentration of our PAA in water, we replot the viscosity data from $\mathrm{Li}$ and Hsieh [43], obtained for the same type of PAA, in log-log scale, together with best power 


\begin{tabular}{|c|c|c|c|c|c|}
\hline $\begin{array}{c}\text { PAA } \\
\text { concentration }(\sim \mathrm{w} / \mathrm{w})\end{array}$ & $\begin{array}{c}\eta \\
(\mathrm{Pa} \cdot \mathrm{s})\end{array}$ & $\begin{array}{c}\mathrm{R} \\
(\mathrm{m}) \times 10^{-4}\end{array}$ & $\begin{array}{c}\mathrm{L} \\
(\mathrm{m})\end{array}$ & $\begin{array}{c}\Delta \mathrm{p} \\
(\mathrm{mbar})\end{array}$ & $\begin{array}{c}\mathrm{Q} \\
(\mathrm{ml} / \mathrm{h})\end{array}$ \\
\hline $10 \%$ & 0.86 & 4.0 & 0.343 & 50 & 0.61 \\
\hline $7.5 \%$ & 0.65 & 4.0 & 0.343 & 40 & 0.65 \\
\hline $7 \%$ & 0.73 & 4.0 & 0.343 & 30 & 0.43 \\
\hline $8.5 \%$ & 0.9 & 4.0 & 0.343 & 20 & 0.23 \\
\hline $11.5 \%$ & 0.85 & 4.0 & 0.343 & 30 & 0.37 \\
\hline
\end{tabular}

Table S2: The measured values of the parameters used to calculate the flow rate.

law fits to these data, in Fig. S2. The linear character with twice the slope at high concentration suggest that $c_{P A A} \approx 5.5 \% \mathrm{w} / \mathrm{w}$ corresponds to the critical entanglement concentration.

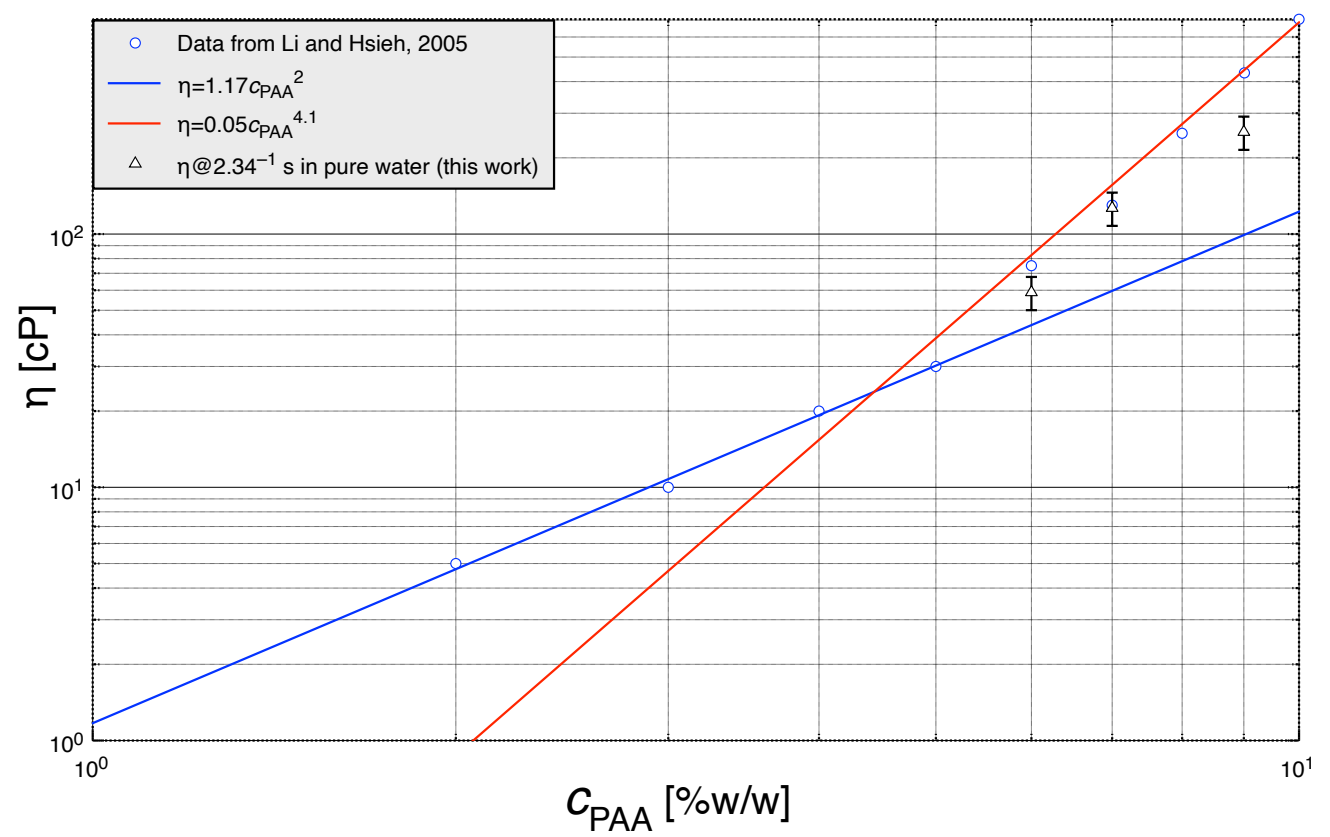

Figure S2: Log-log plot of shear viscosity $\eta$ versus PAA concentration $c_{P A A}$ in water, using experimental data extracted from $\mathrm{Li}$ and Hsieh [43] as well as data from this study. The two power law curves are best fits to the Li and Hsieh data at $c_{P A A}<5.5 \% \mathrm{w} / \mathrm{w}$ and $>5.5 \% \mathrm{w} / \mathrm{w}$, respectively, corresponding to unentangled and entangled solution behavior. The error bars represent a standard deviation obtained from four different measurements, each with increasing and decreasing shear rate. 


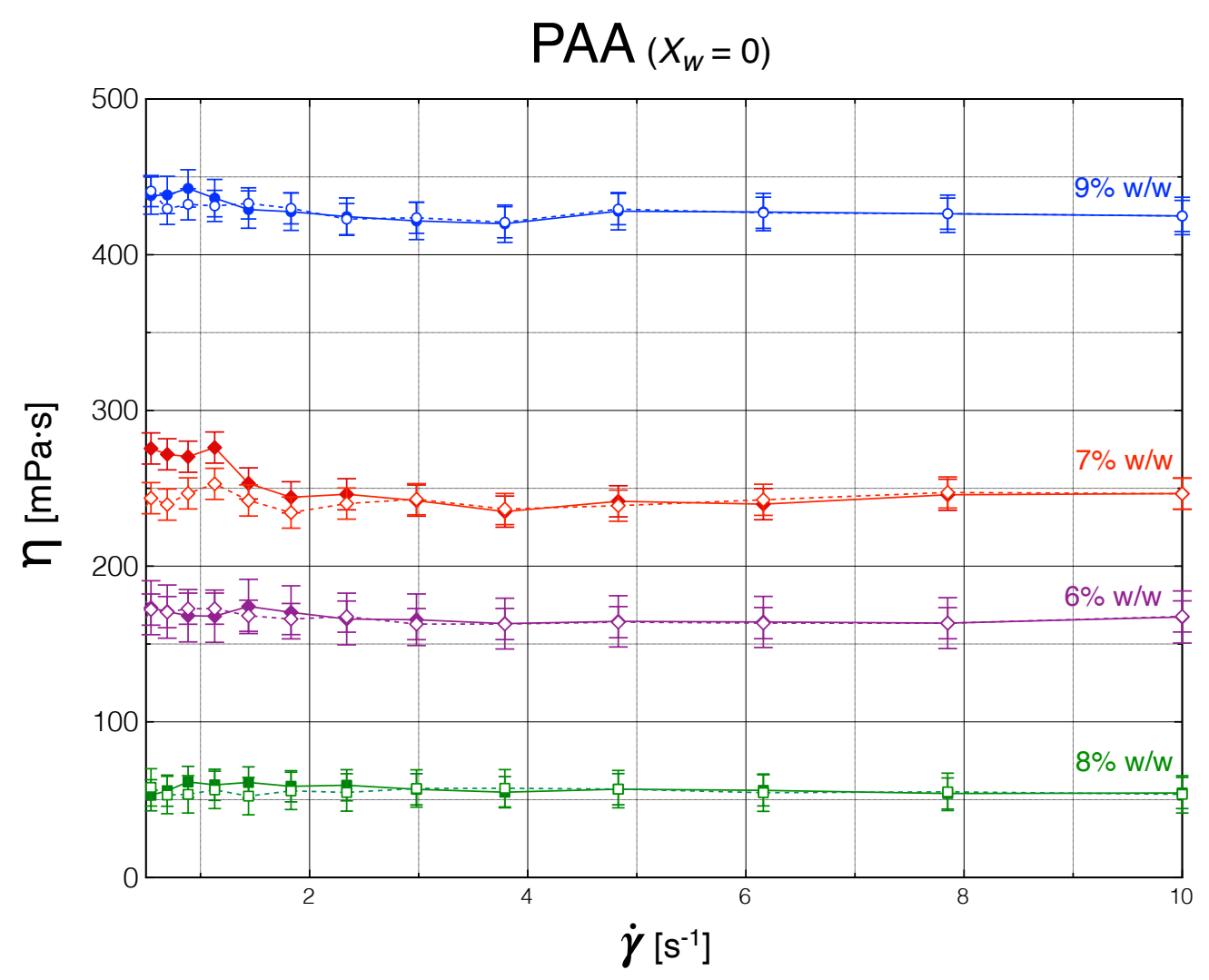

Figure S3: Viscosity $\eta$ as a function of shear rate $\dot{\gamma}$ for different mass concentrations $c_{P A A}$ of PAA, dissolved in pure ethanol. The error bars represent a standard deviation obtained from four different measurements, each with increasing and decreasing shear rate. 


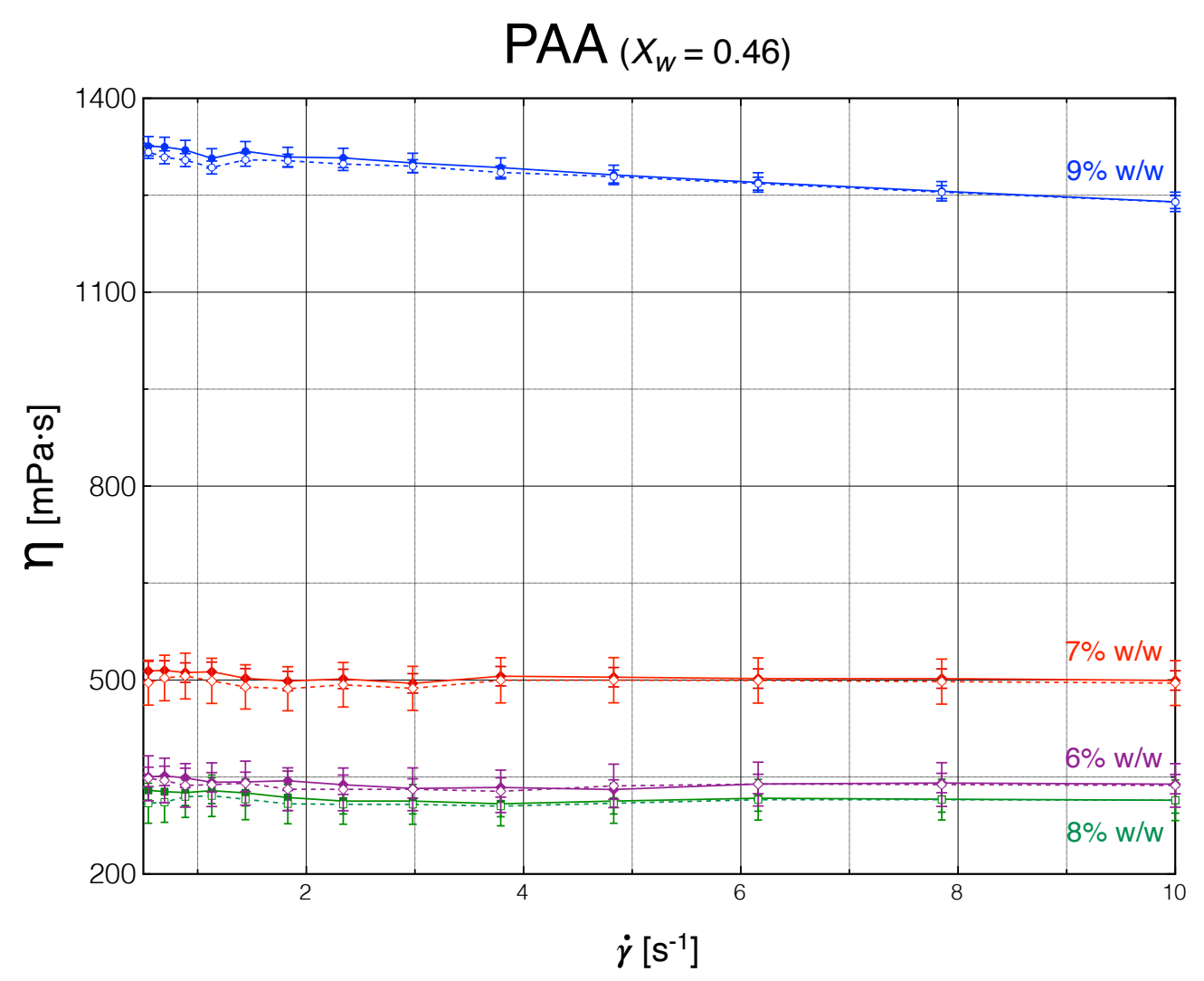

Figure S4: Viscosity $\eta$ as a function of shear rate $\dot{\gamma}$ for different mass concentrations $c_{P A A}$ of PAA, dissolved in a mixture of water and ethanol with $X_{W}=0.46$. The error bars represent a standard deviation obtained from four different measurements, each with increasing and decreasing shear rate.

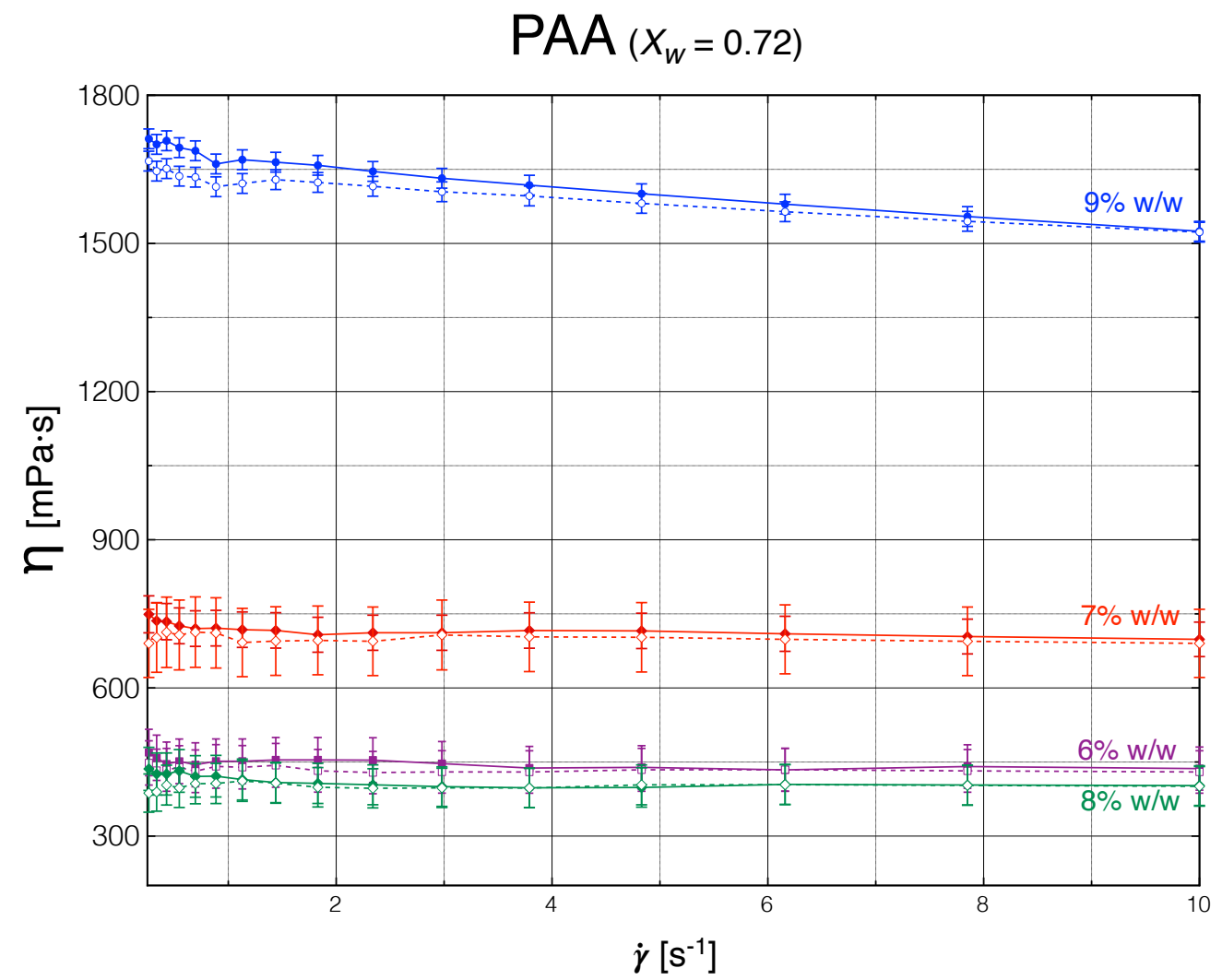

Figure S5: Viscosity $\eta$ as a function of shear rate $\dot{\gamma}$ for different mass concentrations $c_{P A A}$ of PAA, dissolved in a mixture of water and ethanol with $X_{W}=0.72$. The error bars represent a standard deviation obtained from four different measurements, each with increasing and decreasing shear rate. 


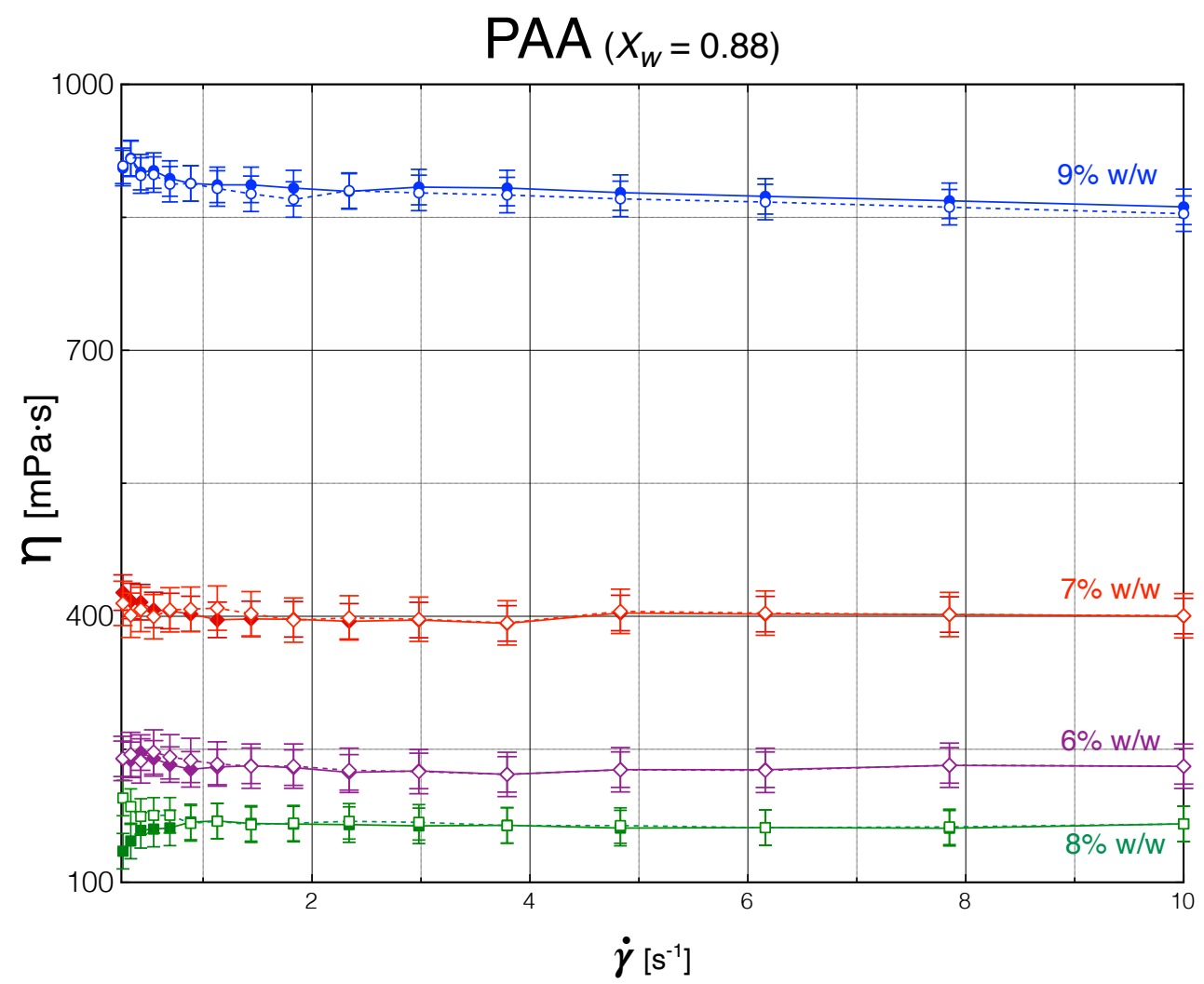

Figure S6: Viscosity $\eta$ as a function of shear rate $\dot{\gamma}$ for different mass concentrations $c_{P A A}$ of PAA, dissolved in a mixture of water and ethanol with $X_{W}=0.88$. The error bars represent a standard deviation obtained from four different measurements, each with increasing and decreasing shear rate.

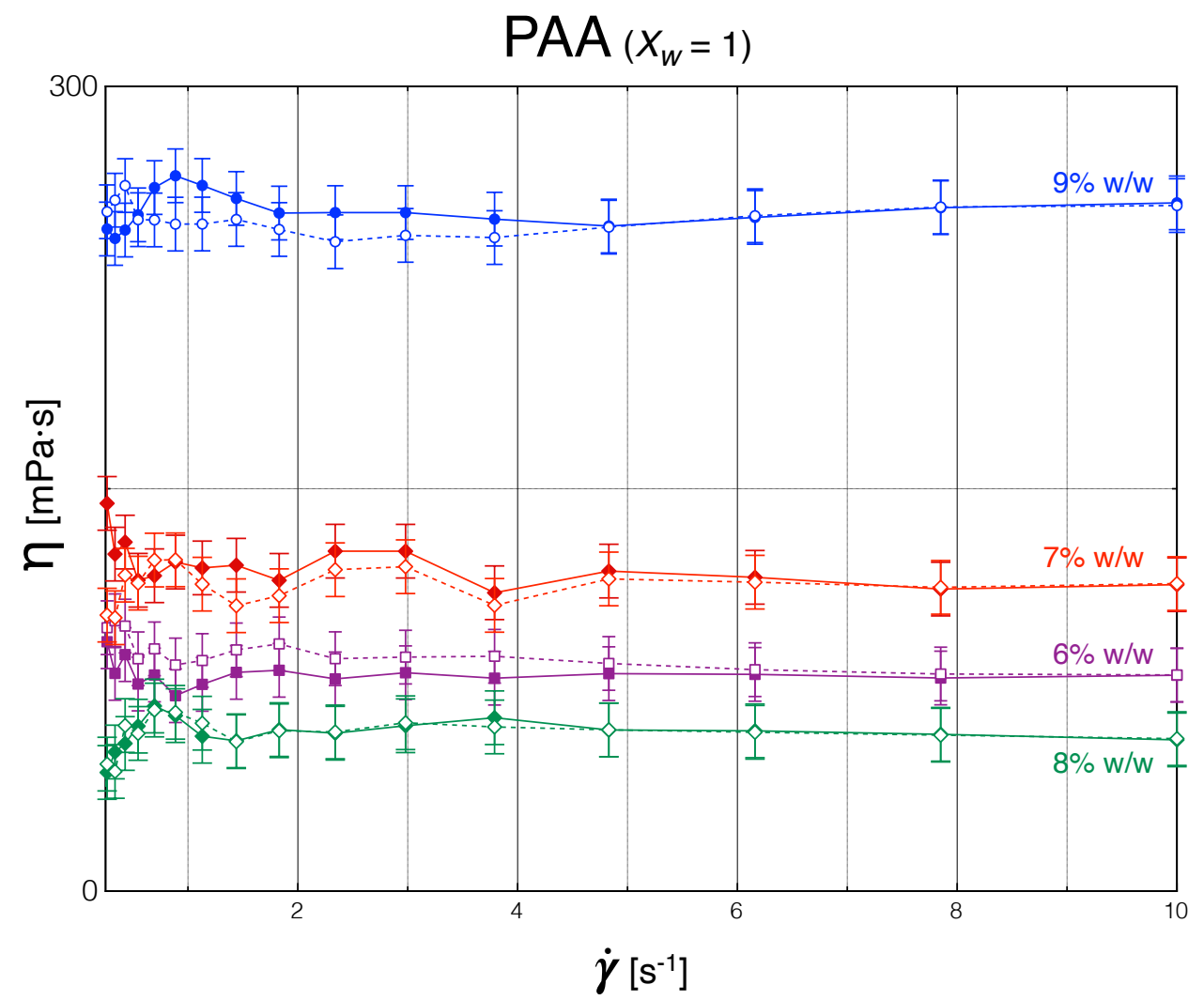

Figure S7: Viscosity $\eta$ as a function of shear rate $\dot{\gamma}$ for different mass concentrations $c_{P A A}$ of PAA, dissolved in pure water. The error bars represent a standard deviation obtained from four different measurements, each with increasing and decreasing shear rate. 


\section{Anomalous 8\% w/w PAA}

The reason that $c_{P A A}=8 \% \mathrm{w} / \mathrm{w}$ is excluded from Fig. 2 in the main paper is that this concentration displayed anomalous behavior during the viscosity measurements. Throughout four attempts we could not get reproducible values, and in one attempt the instrument repeatedly produced negative $\eta$. The data at $\dot{\gamma}=2.34 \mathrm{~s}^{-1}$ from all four attempts, for all five solvent compositions investigated, are shown in Fig. S8, for both increasing and decreasing shear rate $\dot{\gamma}$. We conclude that the PAA solution in this concentration range exhibits a behaviour outside the measurement conditions of the rheometer; hence we do not include them in Fig. 2. A possible reason for the anomaly may be that this particular $c_{P A A}$ range is subject to strong flow-induced phase separation by spinodal decomposition even at the rather low $\dot{\gamma}$ studied here, [62] yielding a highly inhomogeneous sample that the rheometer cannot handle. While the observation is very interesting, its proper elucidation falls outside the scope of the present work.

\section{$8 \% \mathrm{w} / \mathrm{w}$ PAA at $\dot{\gamma} 2.34 \mathrm{~s}^{-1}$}

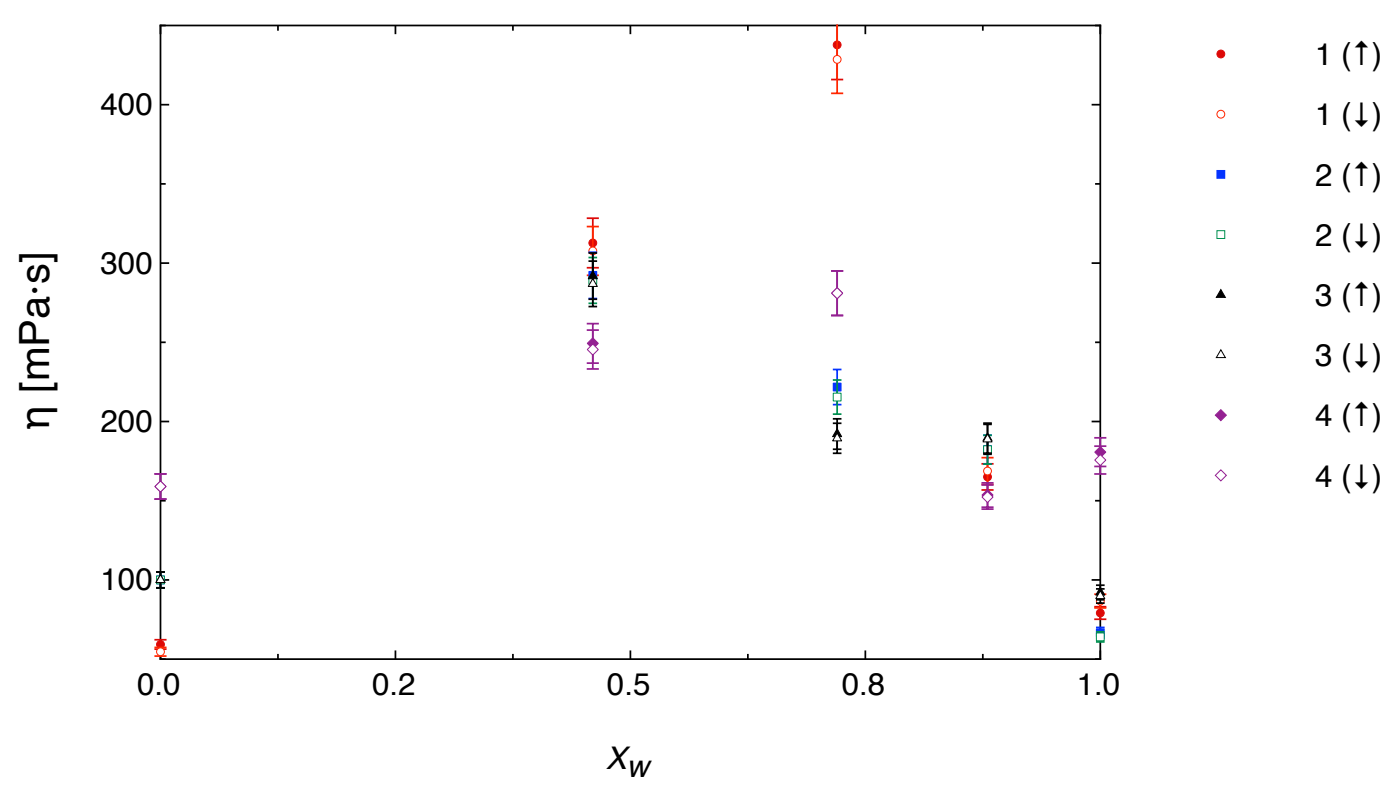

Figure S8: Rheometer output for the viscosity of $8 \% \mathrm{w} / \mathrm{w}$ PAA solutions at a shear rate of $\dot{\gamma}=2.34 \mathrm{~s}^{-1}$, prepared with different water-ethanol ratios. The upwards arrow with solid data points shows increasing shear rate, while the downward arrow with hollowed data points show decreasing shear rate. The error bars represent the standard deviation calculated from six measurements.

\section{Viscosities of solutions optimized for electrospinning}

The variation in viscosity $\eta$ at a shear rate of $\dot{\gamma}=2.34 \mathrm{~s}^{-1}$ for the five solutions tuned to have $\eta \approx \eta_{0}$, making them appropriate for electrospinning regardless of solvent composition, is shown in Fig. S9. The compositions of the solutions are summarized in Table 1 in the main paper. 


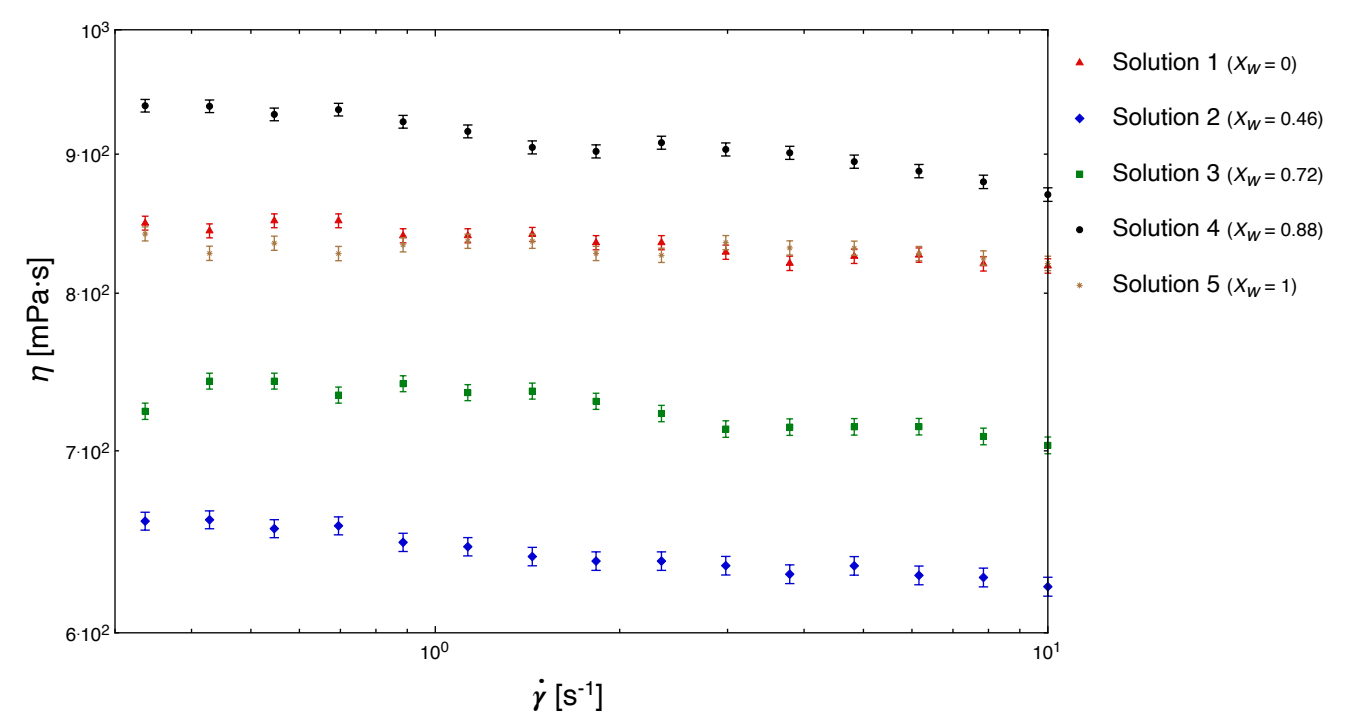

Figure S9: Log-log plot of shear viscosity $(\eta)$ vs. shear rate $(\dot{\gamma})$ for the PAA solutions in Table 1 , with PAA concentration $c_{P A A}$ optimized for constant viscosity regardless of water-ethanol ratio of the solvent mixture. The error bars represent a standard deviation obtained from three different measurements.

\section{E pH measurements}

Fresh PAA solutions of $6 \%, 8 \%$, and $9 \% \mathrm{w} / \mathrm{w}$ were prepared for the $\mathrm{pH}$ measurements which were carried out using Hanna $\mathrm{pH} 211$ Microprocessor $\mathrm{pH}$ meter at room temperature. The $\mathrm{pH}$ values for all the PAA concentration were measured to be lower than 5 .

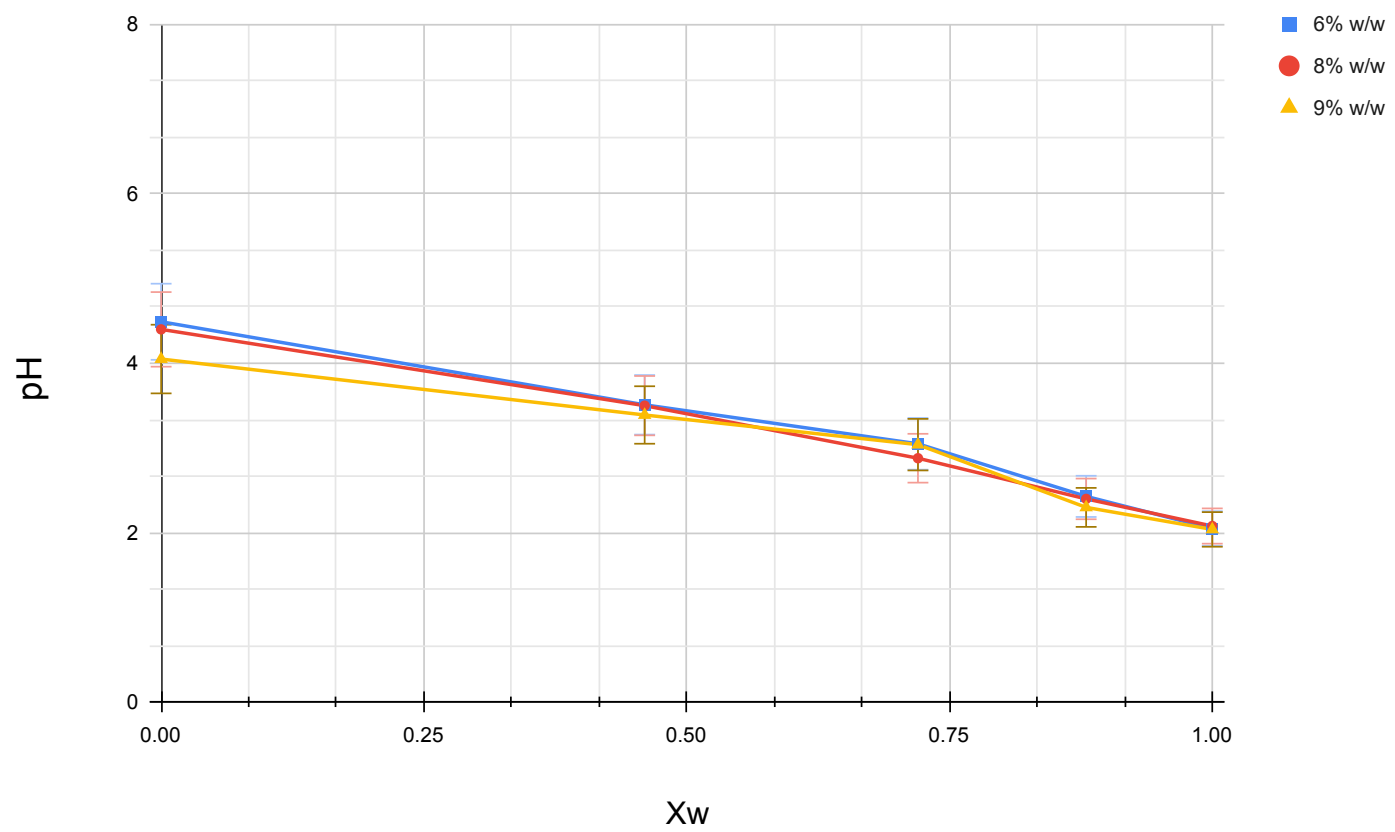

Figure S10: $\mathrm{pH}$ values of $6 \%, 8 \%$, and $9 \%$ PAA in different mass ratios of ethanol and water. The error bars represent a standard deviation obtained from three different measurements. 


\section{F Visual appearance of PAA solutions with different solvent compositions}

Figure S11 shows that solutions of PAA in pure water appear more turbid. As we increase the amount of ethanol in the solution, the turbidity is reduced and the solution becomes clearer (L to R). The samples were imaged after mixing for $30 \mathrm{~s}$ on a vortex mixer.

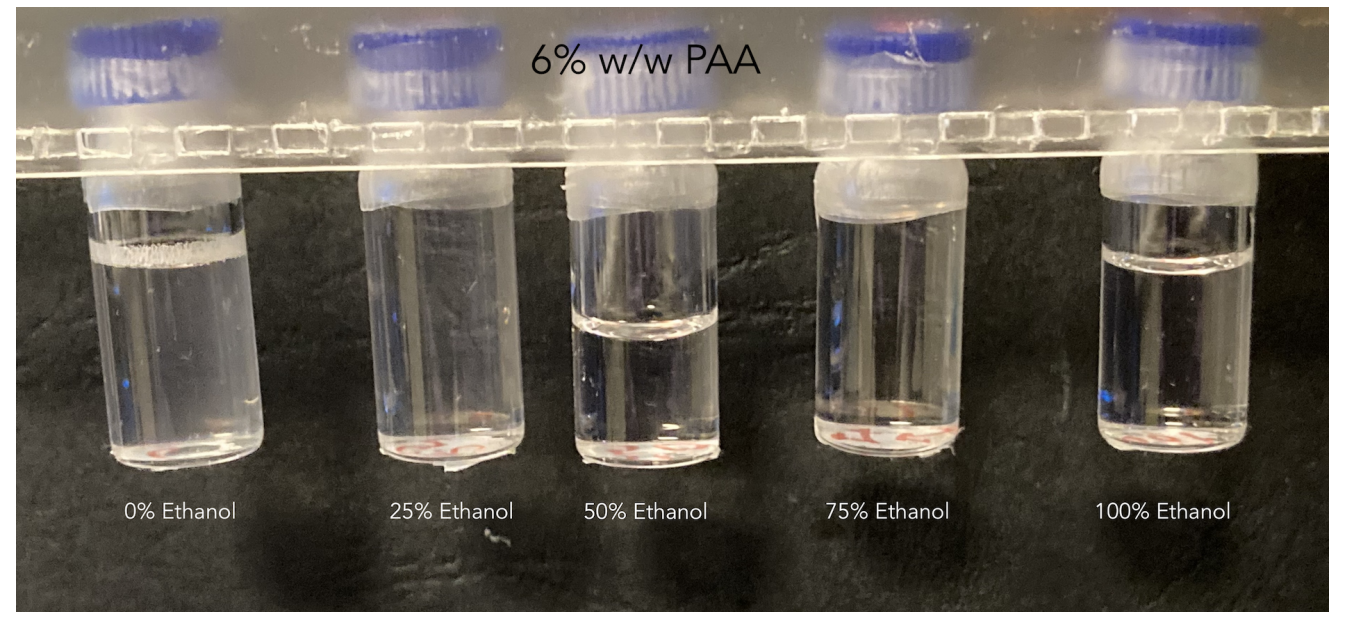

Figure S11: $6 \% \mathrm{w} / \mathrm{w}$ PAA solutions prepared from different ratios of ethanol and water as solvent.

\section{G SI Videos}

SI movie 1: Movie of the Taylor cone recorded during electrospinning of Solution $1\left(X_{w}=0\right)$, as listed in Table 1. The diameter of the spinneret needle is $1.20 \mathrm{~mm}$ and the electrospinning parameters and conditions are listed in the Table S1. This movie corresponds to Figure 3 (a) and (a').

SI movie 2: Movie of the Taylor cone recorded during electrospinning of Solution $2\left(X_{w}=0.46\right)$, as listed in Table 1. The diameter of the spinneret needle is $1.20 \mathrm{~mm}$ and the electrospinning parameters and conditions are listed in the Table S1. This movie corresponds to Figure 3 (b) and (b').

SI movie 3: Movie of the Taylor cone recorded during electrospinning of Solution $3\left(X_{w}=0.72\right)$, as listed in Table 1. The diameter of the spinneret needle is $1.20 \mathrm{~mm}$ and the electrospinning parameters and conditions are listed in the Table S1. This movie corresponds to Figure 3 (c) and (c').

SI movie 4: Movie of the Taylor cone recorded during electrospinning of Solution $4\left(X_{w}=0.88\right)$, as listed in Table 1. The diameter of the spinneret needle is $1.20 \mathrm{~mm}$ and the electrospinning parameters and conditions are listed in the Table S1. This movie corresponds to Figure 3 (d) and (d').

SI movie 5: Movie of the Taylor cone recorded during electrospinning of Solution $5\left(X_{w}=1\right)$, as listed in Table 1. The diameter of the spinneret needle is $1.20 \mathrm{~mm}$ and the electrospinning parameters and conditions are listed in the Table S1. This movie corresponds to Figure 3 (e) and (e'). 
